Aus der Klinik für Thorax-, Herz- und Gefäßchirurgie

(Prof. Dr. med. Dipl.-Phys. F. Schöndube) im Zentrum Chirurgie

der Medizinischen Fakultät der Universität Göttingen

\title{
Die Entwicklung der Durchmesser des pulmonalen Autografts und des linken Ventrikels nach Ross-Operation bei Kindern und Jugendlichen
}

\author{
Inaugural-Dissertation \\ zur Erlangung des Doktorgrades \\ der Medizinischen Fakultät der \\ Georg-August-Universität zu Göttingen
}

vorgelegt von

Melanie Alexandra Harden

aus Wilhelmshaven

Göttingen 2014 
Dekan:

I. Berichterstatter:

II. Berichterstatter/in:

III. Berichterstatter/in:

Tag der mündlichen Prüfung:
Prof. Dr. rer. nat. H. K. Kroemer

Prof. Dr. med. W. Ruschewski

Prof. Dr. med. T. Paul

PD Dr. med. F. Edelmann

18.02.2015 


\section{Inhaltsverzeichnis}

$\begin{array}{lll}\mathbf{1} & \text { Einleitung } & \mathbf{1}\end{array}$

1.1 Die angeborene Aortenklappenstenose . . . . . . . . . . . . . . . . . 2

1.2 Die Therapie der Aortenklappenstenose und -Insuffizienz . . . . . . . . . 4

1.2 .1 Die Kommissurotomie . . . . . . . . . . . . . . . . . . . . . . . . 4

$1.2 .2 \quad$ Die Ballonvalvuloplastie . . . . . . . . . . . . . . . . 4

$1.2 .3 \quad$ Der mechanische Aortenklappenersatz . . . . . . . . . . . . . . 5

1.2.4 Der Aortenklappenersatz durch biologische Implantate . . . . . . 6

1.3 Die Ross-Operation . . . . . . . . . . . . . . . . . . 7

1.3.1 Das Prinzip der Ross-Operation . . . . . . . . . . . . . . . . 7

1.3 .2 Die Indikationen zur Ross-Operation . . . . . . . . . . . . . . . . 8

1.3.3 Die Entwicklung der Ross-Operation . . . . . . . . . . . . . . . . 9

1.4 Ziel der vorliegenden Arbeit . . . . . . . . . . . . . . . . . . . . . . . . 12

2 Patienten und Methoden 13

2.1 Studienaufbau . . . . . . . . . . . . . . . . . . . . . . . . . 13

2.1 .1 Einschlusskriterien . . . . . . . . . . . . . . . . . . . . . . . 13

2.2 Patienten . . . . . . . . . . . . . . . . . . . . . 14

2.3 Operationstechnik . . . . . . . . . . . . . . . . . . . 15

2.4 Die transthorakale Echokardiographie . . . . . . . . . . . . . . . . . 17

2.5 Methodik der statistischen Auswertung . . . . . . . . . . . . . . . . . . . 21

$2.5 .1 \quad$ Z-Werte als Vergleichsinstrument . . . . . . . . . . . . . . . . . . 21

2.5 .2 Das Modell der Z-Wert-Berechnung nach Daubeney . . . . . . . . 22

2.5.3 Das Modell der Z-Wert-Berechnung nach Pettersen . . . . . . . . 23

2.5 .4 Der t-Test für verbundene Stichproben . . . . . . . . . . . . . . . 24

\begin{tabular}{lll}
\hline 3 & Ergebnisse & 25
\end{tabular}

3.1 Operation . . . . . . . . . . . . . . . . . . . . 25

3.1.1 Operationszeiten . . . . . . . . . . . . . . . . 25

$3.1 .2 \quad$ Morphologie der Aortenklappe . . . . . . . . . . . . . . . . . . 25

3.2 Frühmorbidität und -letalität . . . . . . . . . . . . . . . . 25

3.3 Spätmorbidität und -letalität . . . . . . . . . . . . . . . 26

3.4 Die Ergebnisse der echokardiographischen Nachuntersuchungen des $\mathrm{Au}^{-}$ tografts . . . . . . . . . . . . . . . . . 26

$3.4 .1 \quad$ Regressionskurven und Z-Werte nach Daubeney et al. (1999) . . . 28 
3.4.2 Regressionskurven und Z-Werte nach Pettersen et al. (2008) . . . 34

3.5 Die Ergebnisse der echokardiographischen Nachuntersuchungen des linken Ventrikels ............................. 40

$3.6 \quad$ Die Langzeitentwicklung der Homografts und Xenografts . . . . . . . . . 47

4 Diskussion $\quad 51$

4.1 Morbidität und Letalität . . . . . . . . . . . . . . . . . . . . 51

$4.2 \quad$ Die postoperative Entwicklung des pulmonalen Autografts . . . . . . . . 55

4.3 Die postoperative Entwicklung des linken Ventrikels . . . . . . . . . . . . 60

4.4 Die Entwicklung des Homografts/Xenografts als Pulmonalklappensubstitut 62

$\begin{array}{lll}5 & \text { Zusammenfassung } & 67\end{array}$

$\begin{array}{lr}\text { Literatur } & 69\end{array}$

$\begin{array}{ll}\text { Literaturverzeichnis } & 76\end{array}$ 


\section{Tabellenverzeichnis}

$1 \quad$ Allgemeine Patientendaten zum Operationszeitpunkt . . . . . . . . . . . 14

$2 \quad$ Vor der Ross-Operation durchgeführte Eingriffe . . . . . . . . . . . . . . 15

3 Operationstechnik und Operationscharakteristika . . . . . . . . . . . 17

$4 \quad$ Echokardiographische Einteilung der AS nach Bonow et al. (2006) . . . . 19

$5 \quad$ Echokardiographische Einteilung der PS nach Bonow et al. (2006) . . . . 20

$6 \quad$ Echokardiographische Einteilung der Aortenklappeninsuffizienz nach Bonow et al. (2006) . . . . . . . . . . . . . . . . . . . . . . . 21

7 Echokardiographische Einteilung der Pulmonalklappeninsuffizienz nach Bonow et al. (2006) . . . . . . . . . . . . . . . . . . . . 21

$8 \quad$ Art der Komplikation . . . . . . . . . . . . . . . . . . . . 26 


\section{Abbildungsverzeichnis}

$1 \quad$ Operationstechnik nach Ross (1967a) $\ldots \ldots \ldots \ldots$

2 Messungen in der parasternal-langen Achse . . . . . . . . . . . . . . 18

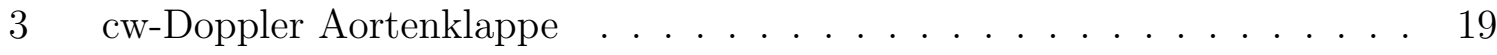

$4 \quad$ Farbkodierte duplexsonographische Messungen in der parasternal-langen

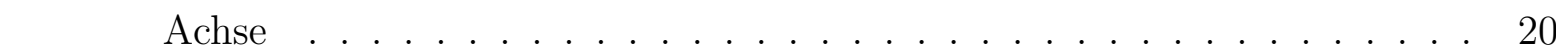

5 Funktion des Autografts in der ersten post-operativen Echokardiographie 27

$6 \quad$ Funktion des Autografts in der zweiten post-operativen Echokardiographie 28

$7 \quad$ Regression nach Daubeney et al. (1999) für den Neo-Aortenklappenring . 29

8 Regression nach Daubeney et al. (1999) für die Neo-Aortenwurzel . . . . 30

$9 \quad$ Regression nach Daubeney et al. (1999) für den sino-tubulären Übergang 31

$10 \quad$ Z-Werte für die Durchmesser von Neo-Aortenklappenring, Neo-Aortenwurzel und sino-tubulärem Übergang nach Daubeney et al. (1999)] . . . . 33

11 Regression nach Pettersen et al. (2008) für den Neo-Aortenklappenring . 35

$12 \quad$ Regression nach Pettersen et al. (2008) für die Neo-Aortenwurzel . . . . . . 36

13 Regression nach Pettersen et al. (2008) für den sino-tubulären Übergang 37

14 Z-Werte für die Durchmesser von Neo-Aortenklappenring, Neo-Aortenwurzel und sino-tubulärem Übergang nach Pettersen et al. (2008)] . . . . . . . . 39

15 Regression nach Pettersen et al. (2008) für den LVESD . . . . . . . . . . 41

$16 \quad$ Regression nach Pettersen et al. (2008) für den LVEDD . . . . . . . . . . 42

17 Regression nach Pettersen et al. (2008) für den Durchmesser des IVS . . 43

18 Regression nach Pettersen et al. (2008) für den Durchmesser der linksventrikulären $\mathrm{HW} \ldots \ldots \ldots \ldots \ldots$. . . . . . . . . . . . 44

19 Z-Werte für LVEDD und LVESD nach Pettersen et al. (2008) . . . . . . 45

$20 \quad$ Z-Werte für IVS und HW nach Pettersen et al. (2008)]. . . . . . . . . . 46

21 Zustand des Homografts/Xenografts in der ersten post-operativen Echokardiographie . . . . . . . . . . . . . . . . . . 47

22 Homograft-/Xenograftstenose in der ersten post-operativen Echokardiographie . . . . . . . . . . . . . . . . . . . . . . . 48

$23 \quad$ Homograft-/Xenograftinsuffizienz in der ersten post-operativen Echokar-

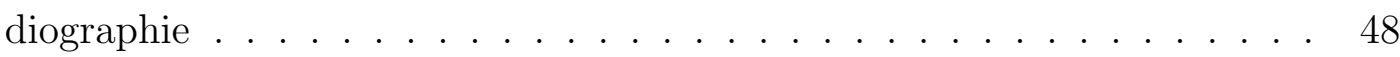

24 Homograft/Xenograft in der zweiten post-operativen Echokardiographie. 49

$25 \quad$ Homograft-/Xenograftstenose in der ersten post-operativen Echokardio-

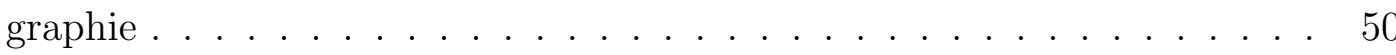


26 Homograft-/Xenograftinsuffizienz in der ersten post-operativen Echokar-

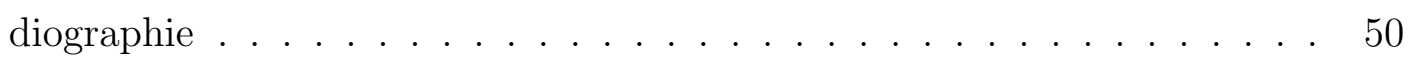




\section{Abkürzungsverzeichnis}

\begin{tabular}{|c|c|}
\hline $\mathrm{AI}$ & Aorteninsuffizienz \\
\hline AKE & Aortenklappenersatz \\
\hline AS & Aortenstenose \\
\hline ASD & atrialer Septum-Defekt \\
\hline AV-Block & atrio-ventrikulärer Block \\
\hline BSA & body surface area \\
\hline BVP & Ballonvalvuloplastie \\
\hline cw-Doppler & continous-wave-Doppler \\
\hline ECMO & extrakorporale Membranoxigenierung \\
\hline EKZ & extrakorporale Zirkulation \\
\hline HIT Typ II & Heparin-induzierte Thrombozytopenie Typ II \\
\hline HW & linksventrikuläre Hinterwand \\
\hline IVS & interventrikuläres Septum \\
\hline $\ln$ & bezeichnet den natürlichen Logarithmus \\
\hline LVEDD & enddiastolischer linksventrikulärer Durchmesser \\
\hline LVESD & endsystolischer linksventrikulärer Durchmesser \\
\hline LVOT & left ventricular outflow tract \\
\hline LVOTO & left ventricular outflow tract obstruction \\
\hline M-mode & motion-mode \\
\hline PK & Pulmonalklappe \\
\hline PKE & Pumonalklappenersatz \\
\hline RVOT & right ventricular outflow tract \\
\hline VSD & Ventrikel-Septum-Defekt \\
\hline
\end{tabular}




\section{Einleitung}

In der Universitätsmedizin Göttingen werden seit dem Jahre 1994 Kinder und Jugendliche, die einen Aortenklappenersatz (AKE) benötigen, mit der sogenannten RossOperation behandelt. Diese Methode, bei der die Aortenklappe durch die eigene Pulmonalklappe (PK) und die PK durch ein Pulmonalis-Homograft (menschliche Spenderklappe) ersetzt werden, hat im Gegensatz zu anderen Verfahren prothetischen Klappenersatzes den Vorteil, dass die Neo-Aortenklappe (Autograft) mitwächst und nach der Operation keine dauerhafte Antikoagulation notwendig ist. Dies ist im Besonderen für Frauen mit Kinderwunsch aufgrund der Teratogenität von Cumarinen und der Thromembolie-Gefahr bei Absetzten der Cumarine vor der Geburt wichtig (Wong et al. 1993). Es stellt aber auch für Kinder, Jugendliche und junge Erwachsene mit hoher körperlicher Aktivität einen entscheidenden Gewinn an Lebensqualität und Sicherheit dar. Die eigene PK als Autograft ist wegen ihrer Morphologie, ihrer Wachstumsfähigkeit und ihrer optimalen hämodynamischen Eigenschaften sowie auf Grund ihrer immunologischen Kompatibilität und der niedrigen Endokarditis-Gefahr ein idealer Ersatz für die geschädigte Aortenklappe (Moidl et al. 2000, Kouchoukos et al. 2004, Elkins et al. 2008).

Die Operationstechnik wurde von Donald N. Ross in den 60er-Jahren entwickelt. Im Jahre 1967 führte er erstmals die Ross-Operation durch und publizierte auch erste Ergebnisse von 14 Patienten (Ross 1967a). Ende der 80er-Jahre wurden die ersten Langzeitergebnisse veröffentlicht (Matsuki et al. 1988) und das Verfahren begann sich auf Grund der guten Resultate nicht nur in der Erwachsenenherzchirurgie, sondern aufgrund seiner oben genannten speziellen Vorteile auch in der Kinderherzchirurgie durchzusetzen. Erste Ergebnisse zeigten zwar, dass sich besonders im ersten Jahr nach der Operation der Durchmesser des Neo-Aortenklappenrings erweiterte (Hokken et al. 1997b), dies jedoch keinen nachweisbar negativen Einfluss auf die Schließfunktion der Klappe (Hokken et al. 1997c) und auf die klinischen und echokardiographischen Ergebnisse hatte (Hokken et al. 1995, Hokken et al. 1997a).

Die Langzeiterfahrungen nach weiteren 20 Jahren haben gezeigt, dass durch die RossOperation bei Kindern und Jugendlichen in verschiedenen Zentren teilweise sehr gute klinische Ergebnisse erzielt wurden (Valeske et al. 2010, Hörer et al. 2009). Andere Studien berichten jedoch sowohl von erhöhten Raten an Stenosen des Homografts in Pulmonalis-Position als auch von einer Insuffizienz des Autografts in Aortenposition, wodurch Reoperationen sowohl des linken als auch des rechten Herzens notwendig wur- 
den (Jonas 2005). Es wird ebenfalls berichtet, dass es in der zweiten postoperativen Dekade zum Auftreten von Dilatationen der Neo-Aortenwurzel kam, die eine Operation des pulmonalen Autografts notwendig machten (Bekkers et al. 2010). Es bleibt abzuwarten, ob sich die bisherigen guten Ergebnisse einiger Zentren, 15 bis 20 Jahre nach der Operation, auch im weiteren Langzeitverlauf bestätigen lassen (Hörer et al. 2010).

\subsection{Die angeborene Aortenklappenstenose}

Bei den in Göttingen mit einer Ross-Operation versorgten Kindern lag als Grunderkrankung in allen Fällen eine angeborene Aortenstenose (AS) vor. Die angeborene AS macht ca. 5\% aller angeborenen Herzfehler aus (Schumacher et al. 2008). Eine solche Stenose gehört zu den Obstruktionen des linksventrikulären Ausflusstraktes (left ventricular outflow tract obstruction = LVOTO). Durch eine Verengung des linksventrikulären Ausflusstraktes (left ventricular outflow tract $=$ LVOT) kommt es zu einer Flussbeschleunigung des ausgeworfenen Blutvolumens und zu einer turbulenten Strömung. Die Klinik, die Art der Behandlung und die Prognose werden zum einen durch die Schwere und Symptomatik der Obstruktion bestimmt, zum anderen durch eventuell assoziierte Fehlbildungen des Herzens oder anderer Organe im Rahmen von Syndromen etc. (Schumacher et al. 2008).

Die AS wird morphologisch nach dem Ort der Obstruktion eingeteilt:

- Die subvalvuläre Aortenstenose bezeichnet eine Engstelle unterhalb der Aortenklappe im Sinne einer LVOTO. Ursache kann z.B. eine fibrinöse Membran oder ein fibromuskulärer Wulst sein. Ebenso kann die gesamte Ausflussbahn tunnelförmig obstruiert sein (Schumacher et al. 2008).

- Die valvuläre Aortenstenose (Aortenklappenstenose) ist eine morphologische Fehlbildung der AK, die zu einer verengten Klappenöffnung führt. Die häufigste Fehlbildung ist die bicuspide Aortenklappe, hier bestehen statt drei Klappensegeln nur zwei. Diese ist mit einem Vorkommen bei 0,9\%-2,0\% der Gesamtbevölkerung die häufigste kongenitale Fehlbildung des Herzens. Bei operationspflichtiger AS wird sie in 20\%-50\% der Fälle angetroffen (Schumacher et al. 2008). Sie ist die führende Ursache der Aortenstenosen im Erwachsenenalter, da sie im Verlauf durch Entzündungen (Endokarditis) und Verkalkung klinisch apparent wird (Swan et al. 1998).

- Die supravalvuläre Stenose liegt oberhalb der Aortenklappe. Dies ist eine seltene 
Form der AS und kann z.B. im Rahmen des Williams-Beuren-Syndroms auftreten (Kotzot et al. 1995).

Die AS kann echokardiographisch anhand der Strömungsgeschwindigkeit bzw. des daraus errechneten Druckgradienten zwischen linkem Ventrikel und Aorta ascendens in drei Schweregrade unterteilt werden (Tabelle 4, Bonow et al. 2006). Bei circa 30\% der betroffenen Kinder besteht zusätzlich eine Aorteninsuffzienz (AI), die Stenose ist jedoch so gut wie immer die führende Komponente (Schumacher et al. 2008).

Die AS führt zu den folgenden Funktionsstörungen und Symptomen:

Da zur Gewährleistung einer ausreichenden Auswurfleistung eine konzentrische muskuläre Hypertrophie des linken Ventrikels entsteht, kommt es bei Belastung durch eine relative koronararterielle Minderversorgung des Myokards zu Angina pectoris, in seltenen Fällen sogar zu Myokardinfarkten. Kann die Auswurfleistung trotz muskulärer Hypertrophie wegen einer Zunahme der Stenose oder Abnahme der Kontraktilität nicht mehr aufrecht erhalten werden, kommt es zu einer Dilatation des linken Ventrikels mit Mitralklappeninsuffizienz und Dyspnoe. Im Verlauf können aufgrund eines passageren Pumpversagens auch Synkopen auftreten. Die betroffenen Kinder haben durch die turbulente Strömung auch ein erhöhtes Risiko für eine Endokarditis (Schumacher et al. 2008).

Die durchschnittliche Lebenserwartung von unbehandelten Patienten, die das erste Lebensjahr überleben, liegt bei ca. 35 Jahren. In einem Kollektiv nicht behandelter Patienten lebten zu Beginn der dritten Lebensdekade noch 60\% der Patienten und zu Beginn der vierten Lebensdekade nur noch 40 \%(Campbell 1968). Da die (strukturellen) myokardialen und pulmonalen Veränderungen mit zunehmender Dauer ihres Bestehens auch bei erfolgreicher Operation immer weniger rückbildungsfähig sind, ist eine rechtzeitige Operation auch asymptomatischer Patienten zwingend dargeboten. Daher kann die symptomatische Therapie bei klinisch manifester AS durch Sportverbot und Endokarditisprophylaxe nur eine Notlösung zur Überbrückung der Zeit bis zu einer kausalen Therapie darstellen. 


\subsection{Die Therapie der Aortenklappenstenose und -Insuffizienz}

\subsubsection{Die Kommissurotomie}

Eine initiale chirurgische Behandlungsmöglichkeit der Aortenklappenstenose stellt die offene Kommissurotomie dar. Sie wurde Anfang der 50er-Jahre entwickelt. Zunächst war es riskant, da in den Anfängen keine extrakorporale Zirkulation (EKZ) zur Verfügung stand. Es musste ein longitudinaler Zugang an der Aorta geschaffen werden, über den nach Einnähen eines künstlichen Tunnels als Operationzugang einzig der Finger des Operateurs zur genauen Platzierung eines Dilatators zur Kommisurensprengung eingebracht wurde (Bailey et al. 1950, Swann et al. 1954). Nach Entwicklung der EKZ und Möglichkeit der Hypothermie (Gilbert et al. 1960) wurde über den linken Ventrikel ein Dilatator zur Spaltung der Komissuren eingeführt (Bahnson et al. 1960).

Heutzutage werden unter EKZ und Infusionskardioplegie die Aorta ascendens eröffnet, die Klappentaschen mobilisiert und die verschmolzenen Kommissuren inzidiert (Brown et al. 2003). Dabei kann es durch Beschädigung der Taschen zur postoperativen Insuffizienz kommen. Die Anwendung der EKZ birgt im Vergleich zur weniger invasiven Ballonvalvuloplastie ein erhöhtes allgemeines Operationsrisiko, insbesondere bei Neugeborenen mit kritischer Aortenstenose. Die Raten von postoperativen AI und Re-AS entsprechen ungefähr denen nach Ballonvalvuloplastie (Justo et al. 1996). Langzeitstudien belegten erwartungsgemäß, dass es sich auf Grund der Re-Stenosierung oder Dilatation im Verlauf um einen palliativen Eingriff handelt, durch den ein Klappenersatz nur aufgeschoben werden kann (Presbitero et al. 1982).

\subsubsection{Die Ballonvalvuloplastie}

Die Ballonvalvuloplastie (BVP), das heißt die perkutane Ballondehnung der Stenose, ist bei Säuglingen und Kindern zur Behandlung der AS aufgrund der raschen Einsetzbarkeit und der geringeren Invasivität in vielen Kliniken insbesondere ohne Herzchirurgie trotz teils besserer Ergebnisse der offenen Kommisurotomie die bevorzugte Behandlungsmethode (Schumacher et al. 2008). Hierbei wird ein Katheter-montierter Ballon in der stenosierten Klappenöffnung platziert und unter Druck entfaltet und die Stenose aufgedehnt. Es können jedoch postinterventionell Reststenosen zurückbleiben oder Re-Stenosierungen auftreten. Ebenso kann die Klappe beschädigt werden. Ein Einriss der Klappentaschen an falscher Stelle kann zu einer schweren AI führen, so dass eine dringliche Indikation zum Ersatz der Klappe bereits im Säuglingsalter resultieren kann (Sholler et al. 1988, Caspi et al. 1994, Hawkins et al. 1996, McCrindle et al. 2001, Pedra 
et al. 2004). Insgesamt sind die Ergebnisse primär meist zufriedenstellend, doch handelt es sich bei diesem Eingriff um eine palliative Therapie, die den notwendigen AKE in ein höheres Alter, idealerweise ins Erwachsenenalter verschiebt (Pedra et al. 2004, Kusa et al. 2004). Nachteil der Ballonvalvuloplastie ist, dass in der Regel nicht die verschmolzene Kommisur, sondern eine Klappentasche an ihrem schwächsten Punkt eingerissen wird, was gelegentlich eine schwere Klappeninsuffizienz mit dringlicher Indikation zum Klappenersatz im Sinne einer Ross-Operation nach sich zieht, die mit einem hohen perioperativen Risiko bei Kleinkindern belastet ist. Neuere Vergleichsstudien zeigen Vorteile für die chirurgische Klappenrekonstruktion (Tirilomis et al. 2013, McElhinney et al. 2005).

\subsubsection{Der mechanische Aortenklappenersatz}

Ein Verfahren zum AKE, welches vor allem bei erwachsenen Patienten Anwendung findet, ist der Einsatz von mechanischen Aortenklappen. Es gibt verschiedene Prothesen aus künstlichen Materialien, die ab 16 mm - 17 mm Außendurchmesser zur Verfügung stehen. Durchgesetzt haben sich heute aufgrund der günstigeren Hämodynamik nahezu ausnahmslos Zweiflügelklappen aus Pyrolite-Carbon (Feuer-gehärtetem Graphit) mit einem Nahtring aus Polyester-Velours. Vor allem bei Kindern birgt dieses Verfahren trotz der praktisch unbegrenzten Haltbarkeit der mechanischen Klappen einige gravierende Nachteile (Alexiou et al. 2000). So ist bei einer mechanischen Herzklappe eine lebenslange Antikoagulation mit Cumarinen notwendig (Bradley et al. 1985). Neben der erhöhten Blutungsgefahr, besonders im Hinblick auf die erhöhte körperliche Aktivität der Kinder (Schumacher et al. 2008), stellen auch ein möglicher späterer Kinderwunsch bei Patientinnen auf Grund der Teratogenität von Cumarinen (Iturbe-Alessio et al. 1986) und des Thrombose- und Blutungsrisikos in der Schwangerschaft ein Argument gegen den mechanischen AKE dar. Außerdem wächst der Patient buchstäblich aus der mechanischen Klappe heraus. Mindestens ein Austausch bis zum Erreichen des Erwachsenenalters ist notwendig. Somit käme eine mechanische Kunstklappe am ehesten für ausgewachsene männliche Jugendliche in Frage (Schumacher et al. 2008). 


\subsubsection{Der Aortenklappenersatz durch biologische Implantate}

In der Frühzeit der Herzklappenchirurgie sind zunächst biologische Materialien verwendet worden.

Grundsätzlich gibt es zwei Typen von biologischen Klappenprothesen:

- Zum einen kann man tierisches Material verwenden (sogenannte Xenografts). Hierfür kommen zum Beispiel Gerüst-montierte Schweineaortenklappen (z.B. Han$\operatorname{cock}^{\circledR}$ ) und Klappen-tragende Rinderjugularvenen (Venpro ${ }^{\circledR} /$ Contegra ${ }^{\circledR}$ ) (Boethig et al. 2007) zur Anwendung. Die Klappen können auf einem Polyesterummantelten Metall- oder Kunstoffgerüst (stented) montiert sein. Alternativ stehen aus Rinderperikard geschneiderte tricuspidale Seminularklappen zur Verfügung, die entweder Gerüst-montiert (stented) oder ohne Gerüst (stentless) implantiert werden (Schumacher et al. 2008).

- Zum anderen kann man menschliche Spenderklappen von Verstorbenen an Stelle der AK und PK implantieren (Homograft/Allograft).

Der Vorteil der biologischen Klappenprothesen ist, dass die Patienten nach der Operation nicht dauerhaft antikoaguliert werden müssen (Moinuddeen et al. 1998). Xenografts sind praktisch unbegrenzt verfügbar, gerade was besonders kleine Durchmesser betrifft, wie sie für Säuglinge und Kleinkinder benötigt werden (Schumacher et al. 2008). Bei all diesen biologischen Implantaten kommt es jedoch auf Grund von immunologischen Reaktionen gegen das körperfremde Gewebe zu einer fortschreitenden Gewebsermüdung, Degeneration, Fibrosierung und Verkalkung (Peterseim et al. 1999). Dieser Verschleiß ist umso rascher, je jünger der Patient ist. Die Haltbarkeit liegt bei Kleinkindern zum Teil nur bei wenigen Monaten bis wenigen Jahren und ist in Aortenposition deutlicher und geringer als in Pulmonalis-Position. Für Kinder und Jugendliche ist ein biologischer AKE deshalb keine Therapieoption. 


\subsection{Die Ross-Operation}

\subsubsection{Das Prinzip der Ross-Operation}

Die Ross-Operation ist ein Verfahren zum AKE, bei dem die gesunde PK des Patienten einschließlich eines proximalen Myokardkragens und des proximalen Pulmonalishaupstamms exzidiert und an Stelle der Aortenklappe eingesetzt wird (Autograft). Morphologisch ist bei Größenkompatibilität die PK als Autograft das ideale Substrat für einen AKE. Im Vordergrund steht die Frage, ob die Semilunarklappe aus dem Niederdrucksystem des Pulmonalkreislaufs den erhöhten Anforderungen des Systemkreislaufs auf Dauer standhält. Im Tiermodell konnte gezeigt werden, dass die PK als vitales körpereigenes Gewebe die Eigenschaft besitzt, sich dem erhöhten linksventrikulären Druck durch histologische Umbauprozesse anzupassen und im proportionalen Verhältnis zum Körperwachstum mitwachsen kann (Neves et al. 1997). Zum Ersatz der umgesetzten PK wird je nach Verfügbarkeit ein Xenograft- oder ein Homograft-Transplantat verwendet.

Liegt ein tunnelförmig stenosierter LVOT im Sinne einer Mehretagenstenose vor, kann die Ross-Operation modifiziert werden (Reddy et al. 1996, van Son et al. 1997). Im Jahre 1975 veröffentlichten zuerst Konno et al. und unabhängig davon kurz darauf Rastan und Koncz die Beschreibung eines neuen Operationsverfahrens unter Erweiterung des linken Ventrikels simultan zum AKE. Durch Inzision des Ventrikelseptums und Erweiterungsplastik der linksventrikulären Ausflußbahn über den Klappenring hinaus bis in die Aorta ascendens hinein mit einem Polyester-Patch kann der LVOT so vergrößert werden, dass die Implantation einer mechanischen Klappenprothese in Erwachsenengröße (23 mm $27 \mathrm{~mm}$ ) auch bei Kindern möglich wird. Die dabei notwendige Inzision des subvalvulären rechten Ventrikels wird mit einem Polyester-patch verschlossen. In Anlehnung an diese Operationstechnik kann eine Mehretagenstenose des LVOT auch mit einem Autograft und der anhängenden Vorderwand des rechten Ventrikels vorgenommen werden. Diese Operation wird als Ross-Konno-Operation bezeichnet (Rastan und Koncz 1975, Konno et al. 1975). Statt Polyester-Patch können auch verschiedene andere körpereigene oder -fremde Materialien (anhängende Muskulatur der Vorderwand des rechten Ventrikels, alternativ Polyester-patch, Gewebe aus Spenderaorta etc.) zum Defekt-Verschluss verwendet (van Son et al. 1997). 


\subsubsection{Die Indikationen zur Ross-Operation}

Die Indikation zur Ross-Operation wird im Kinderherzzentrum Göttingen gestellt, wenn andere weniger invasive Therapieverfahren wie die Ballonvalvuloplastie, die Kommissurotomie und eine Aortenklappenrekonstruktion versagt haben und ein mechanischer Klappenersatz nicht sinnvoll ist oder vom Patienten bzw. seinen Eltern abgelehnt wird. Es dürfen keine Kontraindikationen vorliegen. Zu diesen zählen Bindegewebserkrankungen wie das Marfan-Syndrom, eine Insuffizienz der Pulmonalklappe, eine Fenestration der Klappentaschen, eine bicuspide Pulmonalklappe und die Medianecrosis ErdheimGsell. Daher muss präoperativ echokardiographisch und katheterangiographisch überprüft werden, ob die Pulmonalklappe als Autograft geeignet ist. Trotzdem kann oft erst intraoperativ die Eignung der PK als Autograft sicher festgestellt werden. Auch muss die PK der Größe nach der AK ungefähr entsprechen und darf keine der zuvor genannten anatomischen Fehlbildungen aufweisen. Der Operateur kann letztendlich erst intraoperativ beurteilen, ob die Pulmonalklappe zum Aortenklappenersatz (AKE) geeignet ist, da die Echokardiographie darüber keine sichere Auskunft geben kann. Liegen morphologische Fehlbildungen vor, muss ein alternatives Operationsverfahren zum AKE gewählt werden und vorab mit dem Patienten (oder/und den Eltern) besprochen worden sein. Eine Ross-Operation verbietet sich auf Grund der ursächlichen Fehlbildung in der Struktur der Aortenwand, die auch den Pulmonalishauptstamm einbezieht (de Sa et al. 1999). Bei primärer AI beim Marfansyndrom als strukturelle Aortenwanderkrankung ist der komplette Aortenwurzelersatz zum Beispiel durch einen composite valve graft (mechanische Klappe) die Methode der Wahl (LeMaire und Coselli 1997).

Letztlich muss der Patient in der körperlichen Verfassung sein, einen Eingriff mit EKZ und langem kardioplegischem Herzstillstand überstehen zu können. Die Risiken von möglicherweise auftretenden postoperativen Rhythmusstörungen und eines Septuminfarktes bei Verletzungen des ersten koronaren Septalastes und langen Ischämiezeiten müssen abgewogen werden. Weiterhin besteht das Risiko einer nach Jahren anstehenden Re-Operation des Homografts/Xenografts in Pulmonalis-Position aber auch des Autografts bei Dilatation und Klappeninsuffizienz. Vorteile sind die exzellente Hämodynamik, die minimale Thromogenität und die damit nicht notwendige Dauerantikoagulation und die Infektresistenz, die eine verbesserte Lebensqualität zur Folge haben. 


\subsubsection{Die Entwicklung der Ross-Operation}

Zwischen 1962 und 1967 entwickelte der Herzchirurg Donald N. Ross ein neues Verfahren zum Ersatz der Aortenklappe, bei dem die gesunde Pulmonalklappe des Patienten an Stelle der erkrankten Aortenklappe eingesetzt wird. Als Ersatz für die umgesetzte Pulmonalklappe verwendete er die Aortenklappe eines Verstorbenen (Ross 1967b). Er ging davon aus, dass die köpereigene Pulmonalklappe der ideale AKE sei, da diese keine thrombembolischen Ereignisse verursache und sich den höheren Drücken im LVOT optimal anpassen könne. Die vorher verwendeten Aortenklappen aus Spenderherzen zeigten eine frühzeitige Verkalkung mit daraus resultierender erneuter Stenose oder Insuffizienz (Smith 1967).

Noch im gleichen Jahr veröffentlichte er die postoperativen Ergebnisse der ersten 12 Patienten, die er in dieser Weise mit der später nach ihm benannten Ross-Operation behandelt hatte. Außer bei zwei Patienten, die aufgrund von nicht mit der Operation im Zusammenhang stehenden Ereignissen verstorben waren (Subarachnoidalblutung, Myokardinfarkt), berichtete er gute klinische Ergebnisse. In der Diskussion seiner Ergebnisse artikulierte er die Befürchtung, dass das Problem mit den degenerierenden und verkalkenden Homografts nun auf die rechte Herzseite verlagert worden sei. Es wurde aber auch die Hoffnung geäußert, dass aufgrund der niedrigeren Druckverhältnisse im rechtsventrikulären Ausflusstrakt (RVOT) der Einsatz eines Homograft wesentlich länger haltbar sein würde als im LVOT (Ross 1967a). Die Vorteile des neuen Verfahrens waren somit, dass im Gegensatz zur biologischen Prothese an Stelle der Aortenklappe eine fehlende oder zumindest langsamer fortschreitende Degeneration in Pulmonalis-Position resultierte und im Gegensatz zum mechanischen AKE nicht die Notwendigkeit zur Antikoagulation bestand.

Das Verfahren konnte sich zunächst nicht durchsetzen, da die Operation sehr aufwändig und kompliziert war und die Fachwelt skeptisch war, eine univalvuläre Erkrankung mittels eines bivalvulären Eingriffs zu behandeln. Außerdem wurde eine Dilatation des vermeintlich weniger widerstandsfähigen Autografts in Aortenposition befürchtet (Bechtel et al. 2006). Im Jahre 1988 veröffentlichte die Arbeitsgruppe von D. N. Ross Langzeitergebnisse aus 20 Jahren Erfahrung mit dieser Operationstechnik. Es handelte sich um eine retrospektive Multicenterstudie, die 241 Patienten einschloss. Zu diesem Zeitpunkt hatte es nach dem Jahr 1976 bei den untersuchten Patienten keine frühen postoperativen Todesfälle mehr gegeben und trotz fehlender Antikoagulation waren keine thrombembolischen Ereignisse aufgetreten (Matsuki et al. 1988). In den folgenden Jahren wurden diese Ergebnisse durch eine geringe Rate an Re-Operationen des ersten Patientenkollek- 


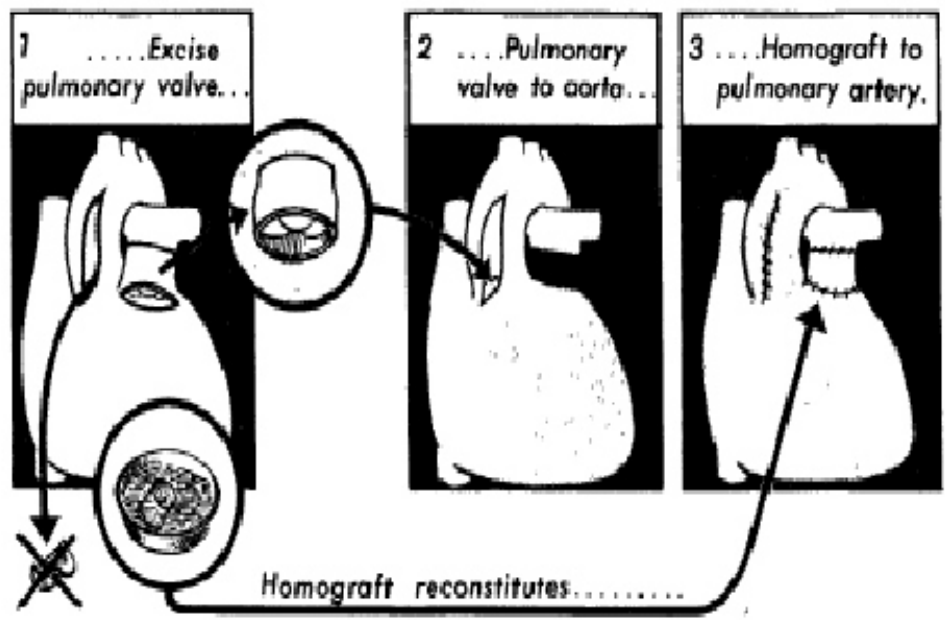

Fig+ 3-5teph in replacement of aortic valve with a pulmonary autoiraft.

Abbildung 1: Operationstechnik nach Ross (1967a)

tivs bestätigt. Die zuvor notwendig gewordenen Re-Operationen wurden mit technischen Defiziten bei der Positionierung der Kommissuren erklärt (Chambers et al. 1997).

Diese guten Ergebnisse und die niedrige Letalität waren Gründe dafür, dass das Verfahren nun auch zur Versorgung von Kindern und Jugendlichen mit angeborenen Fehlbildungen der Aortenklappe herangezogen wurde. Erste Kurzzeitergebnisse wurden Ende der 90er-Jahre veröffentlicht. Diese zeigten eine niedrige Letalität bei guten funktionellen Ergebnissen, selbst nach Voroperationen und bei komplexen Herzfehlern (Marino et al. 1999). Trotzdem wurde weiterhin eine zunehmende Dilatation des Autografts und eine schnelle Degeneration des Homografts mit daraus resultierenden Re-Operationen befürchtet (Walters et al. 1997). So wurde zeitweise sogar von der Ross-Operation als Operationsmethode bei Kindern und Jugendlichen abgeraten, da dies zu erhöhten Raten an Re-Operationen durch massive Dilatationen der Aortenwurzel mit Regurgitationen in den LVOT führen könne (Jonas 2005). Auch die Komplexität des Eingriffs wurde kritisch gesehen. Diese wurde durch den Übergang von der Inklusionstechnik hin zum totalen Ersatz der Aortenwurzel vereinfacht, was aufgrund der besseren Ergebnisse zu einer breiteren Anwendung der Operationsmethode führte (Bechtel et al. 2006).

Seit dem Jahre 2004 erfolgt eine Datenerhebung der in Deutschland und den Niederlanden operierten Patienten im deutschen bzw. europäischen Ross-Register. Hierdurch wird versucht, die Langzeitergebnisse der Ross-Operation zu bewerten, systematisch zu 
erfassen und die bestehenden Fragen zur Haltbarkeit von Autograft und Homograft zu beantworten (Sievers et al. 2004). So suchen aktuelle Veröffentlichungen zu klären, ob der Autograft proportional zum Längenwachstum des Kindes mitwächst oder aber ein im Verhältnis zur Körperoberfläche übermäßiges Wachstum vorliegt (Solymar et al. 2000, Takkenberg et al. 2005, Pasquali et al. 2007a). Die deutschen und internationalen Veröffentlichungen der letzten Jahre zeigen, dass der Bedarf an Re-Operationen des pulmonalen Autografts bei Kindern und Jugendlichen innerhalb der ersten zehn Jahre nach Operation zwar gering ist, aber die Dauer der Zeit nach der Operation der wichtigste Risikofaktor für einen erneut notwendigen AKE bleibt (Hanke et al. 2007, Hörer et al. 2009, Bekkers et al. 2010). 


\subsection{Ziel der vorliegenden Arbeit}

Das Behandlungskonzept von Kindern mit angeborener AS stellt eine große Herausforderung dar, weil die Wahl der Behandlungsmethode sowohl über die Prognose als auch über die Lebensqualität der Kinder entscheidet. Eine rechtzeitige Operation und die Wahl des richtigen Operationsverfahrens ist daher von weitreichender Bedeutung für das weitere Leben des Kindes.

Deshalb ist es von fundamentaler Wichtigkeit, ob es durch Dilatation des Autograft nach Ross-Operation im Langzeitverlauf zu einer behandlungsbedürftigen AutograftInsuffizienz oder Stenose kommt.

Ein weiterer entscheidender Faktor für die Langzeitprognose der Patienten ist die Entwicklung des linken Ventrikels. Studien haben gezeigt, dass es nach AKE zu einem Rückgang der linksventrikulären Masse kommt, die ein Maß für die Hypertrophie des Ventrikels darstellt (Duebener et al. 2005). Biologische Klappen verfügen über bessere hämodynamische Eigenschaften als mechanische Klappen (Pibarot und Dumesnil 2000). Dies führt zu einer geringeren Druckbelastung des Ventrikels. Es ist beschrieben, dass sich aus diesem Grunde nach Ross-Operation eine bestehende Hypertrophie besonders deutlich zurückentwickelt und somit im Langzeitverlauf die Rate sekundärer Myokardschäden durch Hypertrophie geringer ist als bei anderen Verfahren des Klappenersatzes (Duebener et al. 2005, Pasquali et al. 2007a).

Das Ziel der Arbeit war die Darstellung der Ergebnisse der Ross-Operation in Göttingen mit besonderem Augenmerk auf die Entwicklung der Durchmesser des pulmonalen Autografts und des linken Ventrikels. Damit sollte evaluiert werden ob die Ross-Operation die Methode der Wahl des AKE bei Kindern und Jugendlichen darstellt und weiter empfohlen werden kann. 


\section{Patienten und Methoden}

\subsection{Studienaufbau}

Bei der vorliegenden Arbeit handelt es sich um eine retrospektive Studie zur Objektivierung der Operationsergebnisse von 31 Patienten unter 20 Jahren, die von 1994 bis 2008 im Kinderherzzentrum der Universitätsmedizin Göttingen mit der Ross-Operation behandelt wurden. Zur Beurteilung wurden zwei dokumentierte postoperativ durchgeführte echokardiographische Untersuchungen pro Patient herangezogen.

Als Ausgangspunkt diente die erste postoperativ aufgezeichnete Echokardiographie des jeweiligen Patienten. Weil es sich um eine retrospektive Studie handelt und die Echokardiographien im Rahmen der Nachuntersuchungen erfolgten, konnte kein Einfluss auf den Zeitpunkt der Messung und die Vollständigkeit der Dokumentation genommen werden. Da die prä-operativ erstellten Echokardiographien formal nicht vollständig vorhanden waren, wurden sie nicht in die Auswertung mit einbezogen.

Verwendet wurden die auf Video oder Festplatte aufgezeichneten vollständigen Befunde aus den echokardiographischen Nachuntersuchungen. Wir benutzten hierfür jeweils die erste zur Verfügung stehende Untersuchung nach dem Eingriff und die aktuellste Untersuchung (last follow-up). Es konnten nur Echokardiographien für die Studie berücksichtigt werden, die alle für notwendig erachteten Messwerte (siehe Abbildung 2) enthielten. Aufgrund sehr häufig unvollständiger postoperativer Befunderhebungen variiert der Zeitpunkt für die erste verwertbare Echokardiographie zwischen 5 Tagen und 8 Jahren nach der Operation (Mittelwert 397 Tage, Standardabweichung \pm 47 Tage). Als Vergleichsmessung wurden die in der letzten durchgeführten und verwertbaren Kontrollechokardiographie ermittelten Werte herangezogen, um die Entwicklung zu beurteilen. Für die zweite Messung ergab sich ein Nachuntersuchungsintervall zwischen 0,8 und 14 Jahren (= 10 - 168 Monate; Mittelwert 67 Monate, Standardabweichung \pm 96 Monate). Da 10 Patienten nach der Operation in Göttingen ausschließlich am Heimatort kinderkardiologisch nachbetreut wurden, konnten nicht alle Echokardiographien im Kinderherzzentrum Göttingen erstellt werden, sondern wurden vom nachbetreuenden Kinderkardiologen abgefragt.

\subsubsection{Einschlusskriterien}

Eingeschlossen wurden alle Patienten, die zum Zeitpunkt der Operation jünger als 21 Jahre waren und bei denen eine Ross- oder eine Ross-Konno-Operation in der Kinderherzklinik der Universitätsmedizin Göttingen vorgenommen wurde. 
Tabelle 1: Allgemeine Patientendaten zum Operationszeitpunkt der $n=31$ Patienten. Darstellung der Messwerte als Mittelwert \pm Standardabweichung, aufgeteilt nach Geschlecht.

\begin{tabular}{lrr}
\hline Geschlecht & männlich $=22$ & weiblich $=9$ \\
\hline Alter [Monate] & $130,64 \pm 88,83$ & $138,33 \pm 59,61$ \\
Größe $[\mathrm{cm}]$ & $138,59 \pm 40,40$ & $142,11 \pm 23,04$ \\
Gewicht $[\mathrm{kg}]$ & $42,91 \pm 30,37$ & $42,26 \pm 21,22$ \\
Body Mass Index $\left[\frac{\mathrm{kg}}{\mathrm{m}^{2}}\right]$ & $18,84 \pm 4,58$ & $19,59 \pm 5,21$ \\
\hline
\end{tabular}

\subsection{Patienten}

Das Kollektiv setzt sich aus 31 Patienten, 22 männlichen und 9 weiblichen, zusammen. Die Patienten waren zum Operationszeitpunkt zwischen 6 Monaten und 20 Jahren alt (vergleiche Tabelle 1).

Folgende Aortenvitien bestanden prä-operativ:

- 4 Patienten wiesen eine alleinige Aortenklappenstenose auf,

- 4 Patienten hatten eine alleinige Aortenklappeninsuffizienz,

- 23 Patienten besaßen einen kombinierten Aortenklappenfehler.

Intraoperativ zeigte sich bei 15 Patienten eine bicuspidal angelegte und bei den anderen 16 Patienten eine tricuspidal angelegte Aortenklappe. Ein Patient wies zusätzlich zum Aortenvitium eine geringe Mitralklappeninsuffizienz auf.

Vor der Ross-Operation waren bei 25 Patienten (80,6\%) bereits andere Katheter-interventionelle oder offen operative Maßnahmen oder beides am Herzen vorgenommen worden (siehe Tabelle 2). 
Tabelle 2: Vor der Ross-Operation durchgeführte Eingriffe

\begin{tabular}{llc}
\hline Voroperationen* & & Anzahl \\
\hline Eingriffe an der Aortenklappe & Ballonvalvuloplastie & 13 \\
& Kommissurotomie & 13 \\
\hline Eingriffe bei supravalvulärer Stenose & Erweiterungsplastik nach Doty & 2 \\
\hline Eingriffe bei subvalvulärer Stenose & Inzision nach Bigelow & 2 \\
& Septomyektomie nach Morrow & 1 \\
\hline Sonstige Eingriffe & Ventrikel-Septum-Defekt(VSD)-Verschluss & 3 \\
& Aorta ascendens Patch-Plastik & 1 \\
& Aortenbogenrekonstruktion & 1 \\
& Valve shaving & 1 \\
& Verschluss eines Mitralklappenspaltes & 1 \\
& Korrektur des inkompletten AV-Kanals & 1 \\
\hline
\end{tabular}

*zum Teil mehrere Maßnahmen pro Patient

\subsection{Operationstechnik}

Die Ross-Operation wurde bei allen Kindern vom selben Operateur in der Kinderherzklinik Göttingen durchgeführt. Lediglich der erste Eingriff wurde in einer gemeinsamen Operation zusammen mit Professor M. Yacoub (Harefield Hospital of Imperial College, London, Großbritannien) im Rahmen eines Live-Operations-Symposiums vorgenommen. In der Regel erfolgte eine modifizierte Wurzelinklusionstechnik mit Implantation der Koronarostien in die Neo-Aortenwurzel. Vier Kinder wurden mittels vollständiger Wurzelinklusionstechnik versorgt. Hierbei wurde der Autograft in die erhaltene Aortenwurzel eingenäht, nachdem zuvor nur die Klappensegel reseziert wurden. In einem Fall wurde bei einer Patientin davon abweichend eine subkoronare Technik angewandt, bei der der Autograft proximal der Koronarostien (subkoronar) implantiert wurde. Die Ross-Operation wurde bei 12 Patienten aufgrund komplexer Fehlbildungen am Herzen mit anderen Eingriffen kombiniert (siehe Tabelle 3). Im Folgenden ist exemplarisch der vollständige Aortenwurzelersatz erläutert.

Die Operation erfolgte über eine mediane Sternotomie mit Hilfe der extrakorporalen Zirkulation (EKZ). Der Eingriff wurde in tiefer Hypothermie von $24{ }^{\circ} \mathrm{C}$, in einzelnen Fällen in profunder Hypothermie von $18{ }^{\circ} \mathrm{C}$, vorgenommen. Der Herzstillstand wurde durch Injektion kristalloider Kardioplegielösung nach Brettschneider (Custodiol ${ }^{\circledR}$ ) herbeigeführt. Die Injektion erfolgte primär in die Aortenwurzel und wurde alle 30 Minuten selektiv in die Koronarostien wiederholt. Zusätzlich erfolgte eine regelmäßige topische 
Herzkühlung mit $4{ }^{\circ} \mathrm{C}$ kalter Kochsalzlösung. Die aufwändige Myokardprotektion durch tiefe Hypothermie und multiple Kardioplegiedosen erfolgte aufgrund der meist extremen Hypertrophie des linksventrikulären Myokards zur Vermeidung von postoperativen Rhythmusstörungen und einem Pumpversagen des linken Ventrikels.

Nach Mobilisation des Herzens unter totaler EKZ wurde zunächst bei offenen Koronararterien am perfundierten Herzen die PK über eine distale Querinzision des Pulmonalishauptstammes inspiziert. Bikuspidale PK und Klappen mit Fenestrationen in den Taschen, meist in der Nähe der Kommissuren, wurden nicht als Autograft verwendet. Diese Patienten erhielten entsprechend vorheriger Absprache einen konventionellen AKE mit biologischen oder mechanischen Implantaten.

Die Aortenklappe wurde hinsichtlich ihrer Morphologie inspiziert, exzidiert und zur histopathologischen Beurteilung eingeschickt. Kleine Teile wurden zur bakteriologischen Untersuchung eingesandt. Anschließend erfolgte das Einnähen des pulmonalen Autografts und die Re-Implantation der Koronarien in die Sinus des Autografts (proximal mit Einzelknopfnähten Ethibond ${ }^{\circledR}$ 4-0, distal mit fortlaufender Naht Prolene ${ }^{\circledR}$ 5-0). Als letzter Schritt wurde ein Pulmonalishomograft in den RVOT implantiert, unter pro-

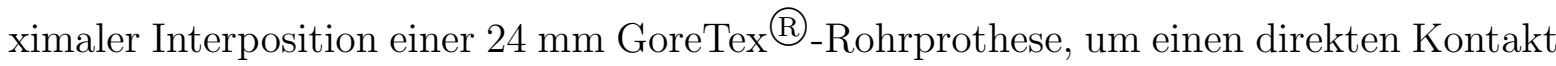
von Spender- und Empfängermyokarden zu vermeiden, die Klappentaschen zum Schutz vor einer Kompression durch das Sternum weit nach distal platzieren zu können und einen strömungsdynamisch optimalen knickfreien Anschluss an den RVOT zu ermöglichen (verwendete Substrate siehe Tabelle 3). 
Tabelle 3: Operationstechnik und Operationscharakteristika

\begin{tabular}{llc}
\hline Operationscharakteristika & & Anzahl \\
\hline Operationstechnik & Wurzelersatz mit modifizierter Wurzelinklusion & 26 \\
& vollständige Wurzelinklusion & 4 \\
& subkoronar & 1 \\
\hline Pulmonalklappenersatz & Pulmonalis-Homograft & 23 \\
& Aorten-Homograft & 4 \\
& porciner Xenograft & 2 \\
& boviner Xenograft & 2 \\
\hline Maßnahmen gegen Dilatation des & Polyester-Prothese & 3 \\
Autografts & Gore-Tex-Prothese & 2 \\
\hline begleitende Eingriffe am LVOT & Rastan-Konno-Operation & 2 \\
& Septomyektomie nach Morrow & 2 \\
& subvalvuläre Inzision nach Bigelow & 2 \\
\hline andere begleitende Eingriffe & atrialer Septum-Defekt (ASD) Verschluss & 1 \\
& Ductus arteriosus-Verschluss & 1 \\
& Schrittmacherwechsel & 1 \\
\hline
\end{tabular}

\subsection{Die transthorakale Echokardiographie}

Die verwendeten Echokardiographiegeräte sind von der Firma Philips (Deutsche Vertretung Hamburg). Verwendet wurden die Geräte:

- Sonos 5500 mit den Schallköpfen S3, S8 und S12 und

- iE33 mit den Schallköpfen S5, S8 und S12

Gemessen wurden folgende Durchmesser des linken Ventrikels im M-mode (motionmode):

- endsystolischer Ventrikeldurchmesser (LVESD)

- enddiastolischer Ventrikeldurchmesser (LVEDD)

- Dicke des Ventrikelseptums in Diastole (IVS)

- Dicke der linksventrikulären Hinterwand in Diastole (HW). 


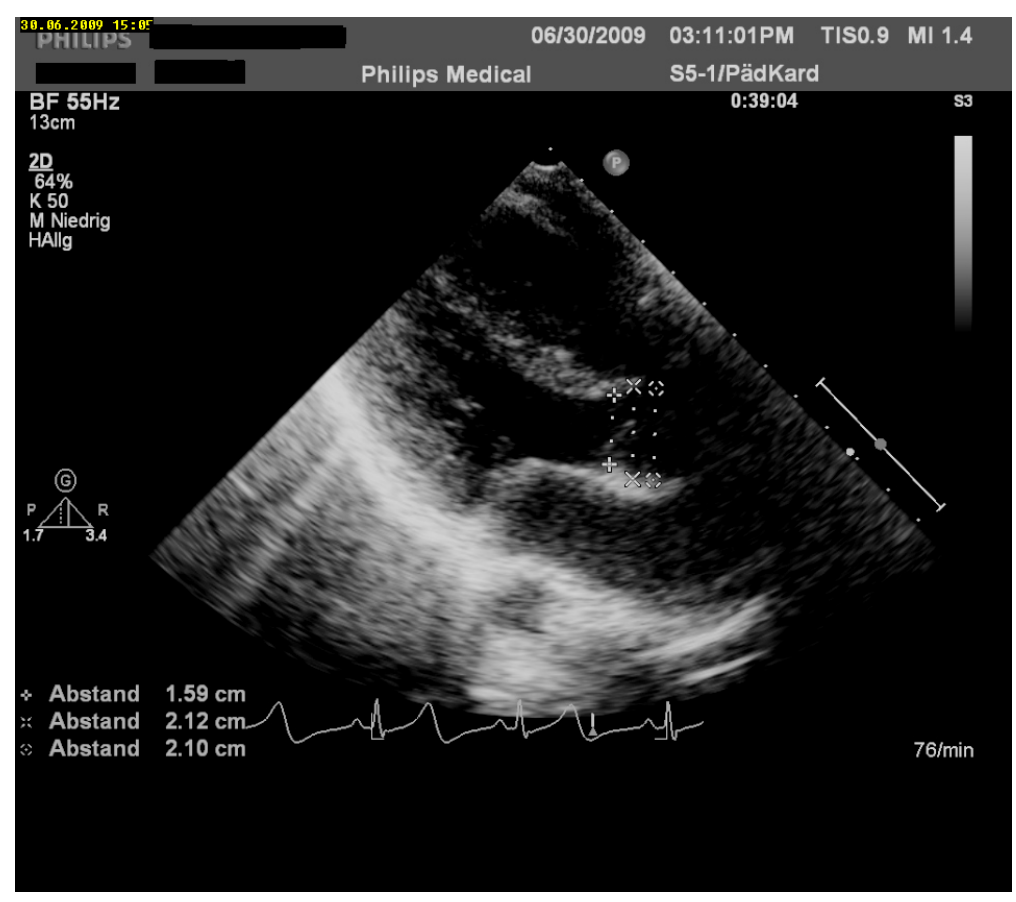

Abbildung 2: Echokardiographische Messungen in der parasternal-langen Achse

In der zweidimensionalen Echokardiographie wurden in der parasternal-langen Achse folgende Durchmesser des LVOT, wie bereits von Pasquali et al. (2007a) beschrieben, gemessen (Abbildung 2):

- des Aortenklappenrings,

- der Aortenwurzel,

- des sino-tubulären Überganges in der Diastole. 
Tabelle 4: Echokardiographische Einteilung der AS nach Bonow et al. (2006)

\begin{tabular}{llll}
\hline Schweregrad & $\begin{array}{l}\text { mild } \\
\text { Grad I }\end{array}$ & $\begin{array}{l}\text { moderat } \\
\text { Grad II }\end{array}$ & $\begin{array}{l}\text { schwer } \\
\text { Grad III }\end{array}$ \\
\hline geschätzter Druckgradient $[\mathrm{mmHg}]$ & $<25$ & $25-40$ & $>40$ \\
Jet-Geschwindigkeit $[\mathrm{m} / \mathrm{s}]$ & $<3$ & $3-4$ & $>4$ \\
\hline
\end{tabular}

Die Flussgeschwindigkeiten über Aortenklappe und Pulmonalklappe wurden mittels continuous-wave-Doppler (cw-Doppler) bestimmt (Abbildung 3). Mit Hilfe der Richtlinien der American Heart Association wurde beurteilt, ob eine Stenosierung der Klappen vorlag und welchem Schweregrad diese, wenn vorhanden, zuzuteilen war (Tabelle 4).

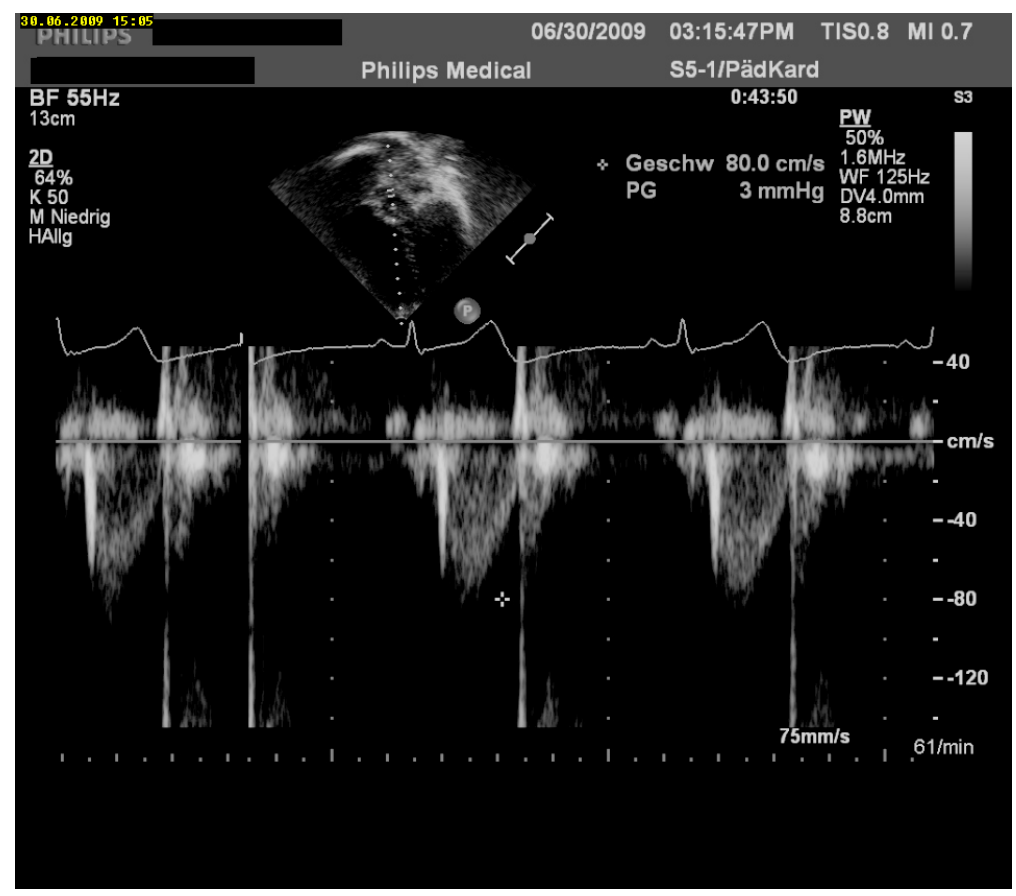

Abbildung 3: cw-Doppler an der Aortenklappe im 3-Kammer-Blick: kein Gradient 
Tabelle 5: Echokardiographische Einteilung der PS nach Bonow et al. (2006)

\begin{tabular}{llll}
\hline Schweregrad & $\begin{array}{l}\text { mild } \\
\text { Grad I }\end{array}$ & $\begin{array}{l}\text { moderat } \\
\text { Grad II }\end{array}$ & $\begin{array}{l}\text { schwer } \\
\text { Grad III }\end{array}$ \\
\hline geschätzter Druckgradient $[\mathrm{mmHg}]$ & $<40$ & $40-60$ & $>60$ \\
Jet-Geschwindigkeit $[\mathrm{m} / \mathrm{s}]$ & $<3$ & $3-4$ & $>4$ \\
\hline
\end{tabular}

Mittels farbkodierter Duplexsonographie (FKDS) wurde bestimmt (Abbildung 4), ob eine Insuffizienz der Neo-Aortenklappe oder des Homografts/Xenografts vorlag. Die echokardiographische Bewertung erfolgte anhand der Regurgitationsfraktion (vergleiche Tabelle 4, Tabelle 5) nach den Kriterien der American Heart Association (Bonow et al. 2006).

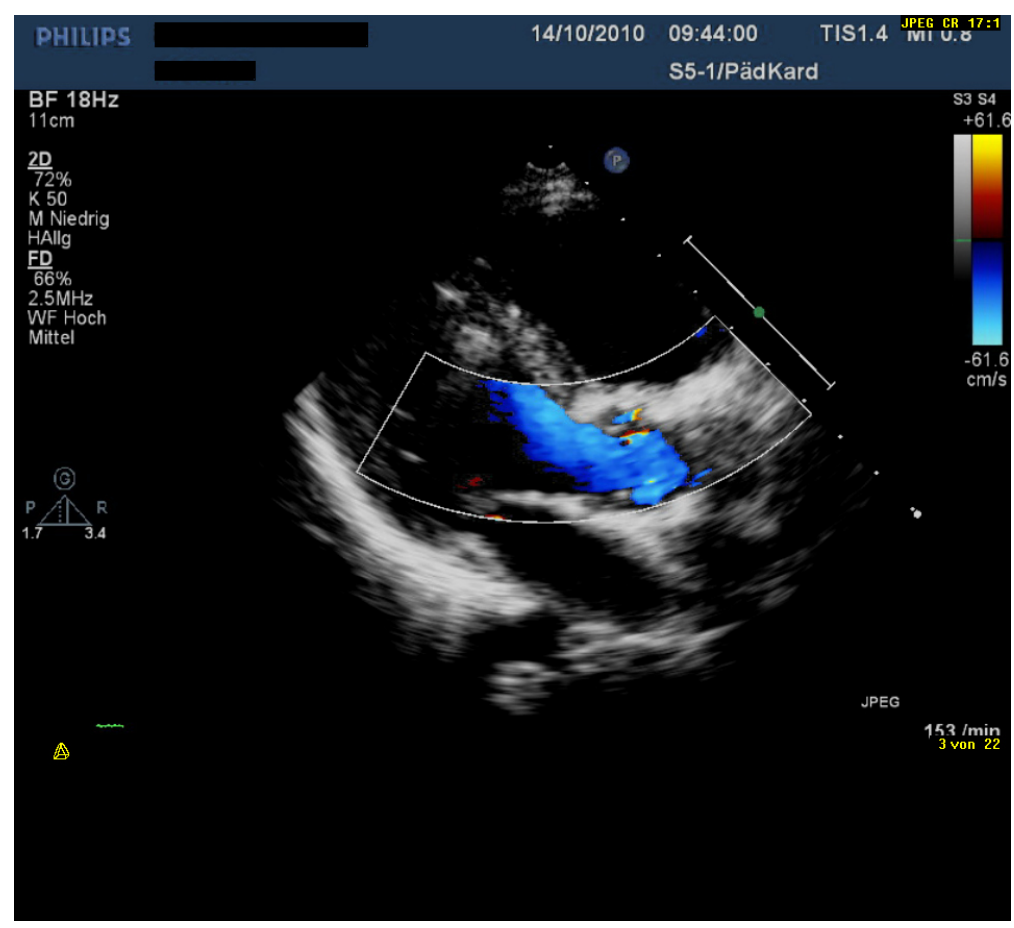

Abbildung 4: Farbkodierte duplexsonographische Messungen in der parasternal-langen Achse 
Tabelle 6: Echokardiographische Einteilung der Aortenklappeninsuffizienz nach Bonow et al. (2006)

\begin{tabular}{llll}
\hline Beurteilung & $\begin{array}{l}\text { mild } \\
\text { Grad I }\end{array}$ & $\begin{array}{l}\text { moderat } \\
\text { Grad II }\end{array}$ & $\begin{array}{l}\text { schwer } \\
\text { Grad III - IV }\end{array}$ \\
\hline Regurgitationsfraktion [\%] & $<30$ & $30-60$ & $>60$ \\
Regurgitationsvolumen [ml] & $<30$ & $30-50$ & $>50$ \\
\hline
\end{tabular}

Tabelle 7: Echokardiographische Einteilung der Pulmonalklappeninsuffizienz nach Bonow et al. (2006)

\begin{tabular}{llll}
\hline Beurteilung & $\begin{array}{l}\text { mild } \\
\text { Grad I }\end{array}$ & $\begin{array}{l}\text { moderat } \\
\text { Grad II }\end{array}$ & $\begin{array}{l}\text { schwer } \\
\text { Grad III - IV }\end{array}$ \\
\hline Regurgitationsfraktion [\%] & $<30$ & $30-60$ & $>60$ \\
Regurgitationsvolumen [ml] & $<30$ & $30-50$ & $>50$ \\
\hline
\end{tabular}

\subsection{Methodik der statistischen Auswertung}

Die statistische Auswertung der erhobenen Daten geschah mit Unterstützung des Institutes für Medizinische Statistik der Universitätsmedizin Göttingen. Die Auswertungen wurden mit der Statistiksoftware STATISTICA, Version 9.0 und Microsoft Excel XP2003 durchgeführt.

\subsubsection{Z-Werte als Vergleichsinstrument}

Da Messwerte sowohl bei Kindern, Jugendlichen als auch jungen Erwachsenen ermittelt wurden, ist es zunächst von Vorteil, die aus verschiedenen Altersklassen stammenden Werte in Bezug zur Altersnorm und individuellen Konstitution zu bringen.

In früheren Untersuchungen wurde gezeigt, dass bei herzgesunden Kindern die Durchmesser des linken Ventrikels und des LVOT in einem proportionalen Verhältnis zur Körperoberfläche (body surface area = BSA) stehen (Henry et al. 1978, Snider et al. 1984). Im Rahmen der wachstumsbedingten Zunahme der BSA kommt es daher ebenfalls zu einer Zunahme der linksventrikulären und aortalen Durchmesser (Roman et al. 1989, Daubeney et al. 1999, Zilberman et al. 2005).

In den Arbeiten von Daubeney et al. (1999) und Pettersen et al. (2008) wurden große 
Kollektive herzgesunder Kinder echokardiographisch untersucht und mittels Regressionen Normbereiche für das Verhältnis zwischen LVOT und BSA konstruiert. Die Ergebnisse aus diesen Arbeiten wurden verwendet, um die gemessenen Durchmesser des LVOT und der BSA der kranken Kinder unseres Kollektivs mit Gesunden zu vergleichen.

Um die Messwerte der in Göttingen behandelten Kinderherzen im Vergleich zu gesunden Kinderherzen bewerten zu können, bedienten wir uns der so genannten Z-Werte. Diese bilden den relativen Zusammenhang zwischen BSA und Aortendurchmesser im Verhältnis zur Normalpopulation ab. Sie beschreiben den Abstand eines Messwertes vom erwarteten Mittel in Einheiten der Standardabweichung.

Analog zu den Untersuchungen von Daubeney et al. (1999) und Pettersen et al. (2008), wurden für die Messwerte der frühen und der späten Nachuntersuchungen die Z-Werte ermittelt. Das Regressionsmodell von Daubeney et al. (1999), basierend auf einem Patientenkollektiv von 125 Kindern, wurde als Vergleichskollektiv verwendet, da es in den Veröffentlichungen des deutschen und europäischen Ross-Registers als Referenz verwendet wird.

Ergänzend wurden die in Göttingen erhaltenen Werte auch mit den Ergebnissen von Pettersen et al. aus dem Jahre 2008 verglichen, da dieser für seine Datenzusammenstellung weit mehr Patienten (782) untersuchte und explizit auch Jugendliche mitberücksichtigt hatte. Zudem schlossen Pettersen et al. in ihre Ergebnisse Auswertungen der Durchmesser des linken Ventrikels mit ein, welche in der vorliegenden Arbeit ebenfalls betrachtet und ausgewertet wurden.

\subsubsection{Das Modell der Z-Wert-Berechnung nach Daubeney}

Dem von Daubeney et al. verwendeten Modell liegt ein regressionsanalytischer Ansatz zu Grunde, um den Zusammenhang zwischen Aortendurchmesser und BSA zu bestimmen (Daubeney et al. 1999):

$$
\ln (\varnothing \text { Aorta })=\beta_{0}+\beta_{1} \cdot \ln (\mathrm{BSA})
$$

Die Regressionsparameter $\beta_{0}$ und $\beta_{1}$ werden aus beobachteten Daten geschätzt. Folglich können zur Vorhersage von Aortendurchmessern spezifische BSA-Werte verwendet werden. Mit diesen Regressionsgleichungen können keine direkten Aussagen über ein anderes Patientenkollektiv getroffen werden, dennoch ermöglichen sie einen Vergleich zwischen gesunden Patienten und den in dieser Arbeit eingeschlossenen Patienten nach Ross-Operation. 
Für die betrachteten Aortendurchmesser an den Messpunkten Aortenklappenring, Aortenwurzel und sino-tubulärer Übergang der Aorta ermittelten Daubeney et al. (1999) die folgenden Regressionsgleichungen:

$$
\begin{aligned}
\ln (\varnothing \text { Aortenklappenring }) & =0,5183+0,5347 \cdot \ln (\mathrm{BSA}) \\
& \text { mean square error }=0,06726 \\
\ln (\varnothing \text { Aortenwurzel }) & =0,7224+0,5082 \cdot \ln (\mathrm{BSA}) \\
& \text { mean square error }=0,07284 \\
\ln (\varnothing \mathrm{STJ}) & =0,5417+0,5490 \cdot \ln (\mathrm{BSA}) \\
& \text { mean square error }=0,08656
\end{aligned}
$$

Mit diesen Regressionsgleichungen lässt sich nun zu jedem Patienten der entsprechende Aortendurchmesser durch die BSA schätzen, der mit der in Wirklichkeit gemessenen Struktur (Aortendurchmesser: $\varnothing \widehat{\text { Aorta }}$ ) in folgender Gestalt in Relation gesetzt werden soll:

$$
Z=\frac{\ln (\varnothing \widehat{\text { Aorta }})-\ln (\text { Aorta })}{\sqrt{\text { mean square error }}}
$$

\subsubsection{Das Modell der Z-Wert-Berechnung nach Pettersen}

Einen alternativen Ansatz untersuchten Pettersen et al. (2008). Sie unterstellten ein polynomielles Regressionsmodell der Gestalt:

$$
\ln (\varnothing \text { Aorta })=\beta_{0}+\beta_{1} \cdot \mathrm{BSA}+\beta_{2} \cdot \mathrm{BSA}^{2}+\beta_{3} \cdot \mathrm{BSA}^{3}
$$

Und erhielten mit den von ihnen erhobenen Daten die folgenden Regressionsgleichungen:

$$
\begin{array}{r}
\ln (\varnothing \text { Aortenklappnring })=-0,874+2,708 \cdot \mathrm{BSA}-1,841 \cdot \mathrm{BSA}^{2}+0,452 \cdot \mathrm{BSA}^{3} \\
\text { mean square error }=0,010 \\
\ln (\varnothing \text { Aortenwurzel })=-0,500+2,537 \cdot \mathrm{BSA}-1,707 \cdot \mathrm{BSA}^{2}+0,420 \cdot \mathrm{BSA}^{3} \\
\text { mean square error }=0,012 \\
\ln (\varnothing \mathrm{STJ})=-0,759+2,643 \cdot \mathrm{BSA}-1,797 \cdot \mathrm{BSA}^{2}+0,442 \cdot \mathrm{BSA}^{3} \\
\text { mean square error }=0,018
\end{array}
$$


Die Z-Werte werden analog zu Daubeney et al. (1999) durch die Formel (1) berechnet. Pettersen et. al wendeten ihr Modell auch auf die linksventrikulären Durchmesser an, die standardmäßig in Echokardiographien bestimmt werden:

$$
\begin{aligned}
& \ln (\varnothing \mathrm{LVESD})=-0,371+2,833 \cdot \mathrm{BSA}-2,081 \cdot \mathrm{BSA}^{2}+0,538 \cdot \mathrm{BSA}^{3} \\
& \text { mean square error }=0,016 \\
& \ln (\varnothing \mathrm{LVEDD}) \quad=0,105+2,859 \cdot \mathrm{BSA}-2,119 \cdot \mathrm{BSA}^{2}+0,552 \cdot \mathrm{BSA}^{3} \\
& \text { mean square error }=0,010 \\
& \ln (\varnothing \mathrm{IVS})=-1,242+1,272 \cdot \mathrm{BSA}-0,762 \cdot \mathrm{BSA}^{2}+0,208 \cdot \mathrm{BSA}^{3} \\
& \text { mean square error }=0,046 \\
& \ln (\varnothing \mathrm{HW})=-1,586+1,849 \cdot \mathrm{BSA}-1,188 \cdot \mathrm{BSA}^{2}+0,313 \cdot \mathrm{BSA}^{3} \\
& \text { mean square error }=0,037
\end{aligned}
$$

Somit ist es möglich zusätzlich zu den Durchmessern des LVOT auch die Durchmesser des linken Ventrikels im Vergleich zum gesunden Kollektiv zu beurteilen.

\subsubsection{Der $\mathrm{t}$-Test für verbundene Stichproben}

Mittels t-Tests für verbundene Stichproben wurde schließlich ermittelt, ob sich die ermittelten Z-Werte innerhalb des Nachtuntersuchungszeitraumes signifikant verändert hatten. 


\section{Ergebnisse}

\subsection{Operation}

\subsubsection{Operationszeiten}

Die durchschnittliche Schnitt-Naht-Zeit betrug zwischen 05:05 und 11:04 Stunden (im Mittel 07:34 Stunden). Die Aortenklemmzeit variierte zwischen 01:54 und 04:44 Stunden (im Mittel 03:00 Stunden). Die Perfusionszeit der notwendigen EKZ betrug zwischen 02:55 und 07:48 Stunden (im Mittel 05:05 Stunden).

\subsubsection{Morphologie der Aortenklappe}

Intraoperativ fand sich bei 15 Patienten eine bicuspidal angelegte Aortenklappe, bei 16 Patienten war die Aortenklappe tricuspid.

\subsection{Frühmorbidität und -letalität}

Innerhalb der ersten 30 postoperativen Tage kam es zu einer Reihe von leichten und schwerwiegenden Komplikationen.

Es kam zu einem Frühtodesfall. Ein 13-jähriges Mädchen, das aufgrund eines kombinierten Aortenvitiums operiert worden war, verstarb am zweiten postoperativen Tag. Bei ausgeprägten, medikamentös nicht zu beherrschenden Herzrhythmusstörungen bestand die Indikation zur postoperativen Kreislaufunterstützung durch eine extrakorporale Membranoxygenierung (ECMO). Unter dieser zunächst erfolgreichen Therapie kam es zu einer massiven Hirnblutung und zu den klinischen und apparativen Zeichen des Hirntods, so dass die Therapie eingestellt wurde. Als Ursache am wahrscheinlichsten war eine Heparin-induzierte Thrombozytopenie Typ II (HIT Typ II), da ein massiver Thrombozytenabfall dokumentiert werden konnte. Auf Wunsch der Angehörigen wurde auf eine Obduktion verzichtet, so dass die Todesursache nicht vollständig geklärt werden konnte.

Die weiteren behandlungsbedürftigen Frühkomplikationen sind in Tabelle 8 aufgezählt. Diese konnten nahezu alle medikamentös behandelt werden. Es kam bei keinem der Patienten zu nachweisbar bleibenden Schäden. 
Tabelle 8: Art der Komplikation

\begin{tabular}{lc}
\hline Komplikation & Anzahl \\
\hline Immunologische Reaktion auf den Homograft & 5 \\
Pneumonie & 3 \\
Atrio-ventrikulärer Block(AV-Block) III. Grades & 1 \\
Blutung nach Ziehen des links-atrialen Druckmesskatheters & 1 \\
Pleuraerguss & 1 \\
Subduralblutung & 1 \\
Harnverhalt & 1 \\
Stressulkus & 1 \\
\hline
\end{tabular}

\subsection{Spätmorbidität und -letalität}

Während des beschriebenen Nachuntersuchungszeitraumes traten keine Spättodesfälle auf. Bei 1 Patienten, bei dem aufgrund eines AV-Block III. Grades ein Herzschrittmacher implantiert worden war, wurde im Verlauf ein Aggregatwechsel und eine Sondenrevision notwendig. 1 Patient ist nach der Operation unbekannt verzogen, so dass von ihm kein zweites postoperatives Echokardiogramm oder Aufzeichnungen über die Spätmorbidität vorlagen (lost to follow-up).

\subsection{Die Ergebnisse der echokardiographischen Nachuntersuchungen des Autografts}

Die funktionellen postoperativen Ergebnisse der Neo-Aortenklappen waren insgesamt sehr gut. In der ersten postoperativen echokardiographischen Nachuntersuchung zeigte sich bei insgesamt 20 von 30 operierten Patienten eine normale Klappenfunktion des Autografts (Abbildung 5). Bei 8 Patienten war eine klinisch nicht relevante Insuffizienz ersten Grades nachzuweisen. Bei 1 Patienten wurde eine leichtgradige valvuläre Stenose mit einer Jet-Geschwindigkeit von 2,2 m/s (entsprechend einem Druckgradienten von 19 $\mathrm{mmHg}$ ) festgestellt, von diesem Patienten stand aufgrund eines Umzuges in der Folge keine weitere Echokardiographie zur Verfügung (lost to follow-up). 1 Patientin wies eine moderate bis schwere supravalvuläre Aortenstenose auf mit einer Jet-Geschwindigkeit von $4 \mathrm{~m} / \mathrm{s}$ (entsprechend $64 \mathrm{mmHg}$ ), bei unauffälligen Flussgeschwindigkeiten und Drücken im Bereich des Autografts auf $(1 \mathrm{~m} / \mathrm{s}$ entsprechend $4 \mathrm{mmHg})$. Im weiteren Verlauf er- 
gaben sich bei dieser Patientin in den zur Verfügung stehenden Untersuchungen keine echokardiographischen Auffälligkeiten mehr.

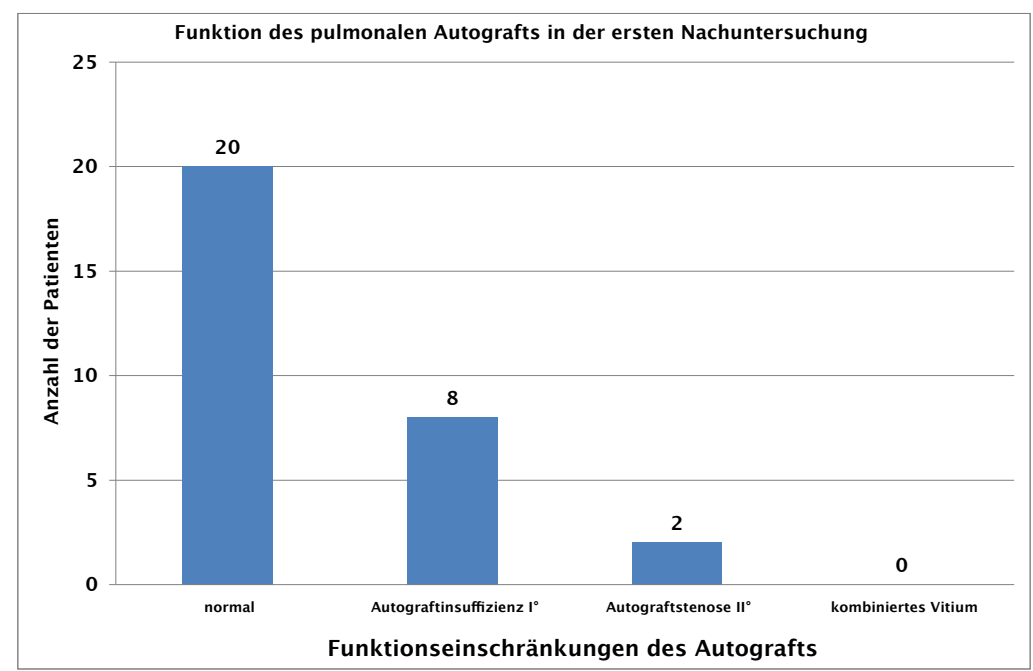

Abbildung 5: Funktion des Autografts in der ersten post-operativen Echokardiographie ( $\mathrm{n}=30$ Patienten, da 1 Patientin verstorben)

Im betrachteten Zeitraum musste bei einem Patienten nach 12 Jahren simultan mit dem Austausch des degenerierten Homografts in Pulmonalisposition die Kürzung des pulmonalen Autografts und Ummantelung der erweiterten Neo-Aortenwurzel durchgeführt werden. Bei diesem Patienten hatten präoperativ eine bicuspid angelegte Aortenklappe und Stenosen auf supravalvulärer, valvulärer und subvalvuläer Ebene bestanden. Diese waren im Kleinkindalter zunächst mittels offener Kommissurotomie und Septomyektomie nach Morrow behandelt worden. Die Ross-Operation war als kompletter Wurzelersatz durchgeführt worden. Der postoperative Verlauf hatte keine Auffälligkeiten gezeigt. In der ersten postoperativen Echokardiographie hatten keine Stenosen bestanden. Im nachbeobachteten Zeitraum war es zu einer Stenosierung des Autografts an der distalen Anastomose gekommen.

Bei keinem anderen Patienten musste eine Korrektur der Autograftklappe erfolgen.

In der letzten echokardiographischen Nachuntersuchung bestand bei insgesamt $12 \mathrm{~Pa}-$ tienten noch immer eine uneingeschränkte Funktion des Autografts, bei 15 Patienten wurde eine klinisch nicht relevante Autograftinsuffizienz ersten Grades festgestellt. 1 Patient wies zusätzlich eine geringe subvalvuläre Stenose $(2,2 \mathrm{~m} / \mathrm{s})$ des pulmonalen $\mathrm{Au}-$ 
tografts auf (Abbildung 6).

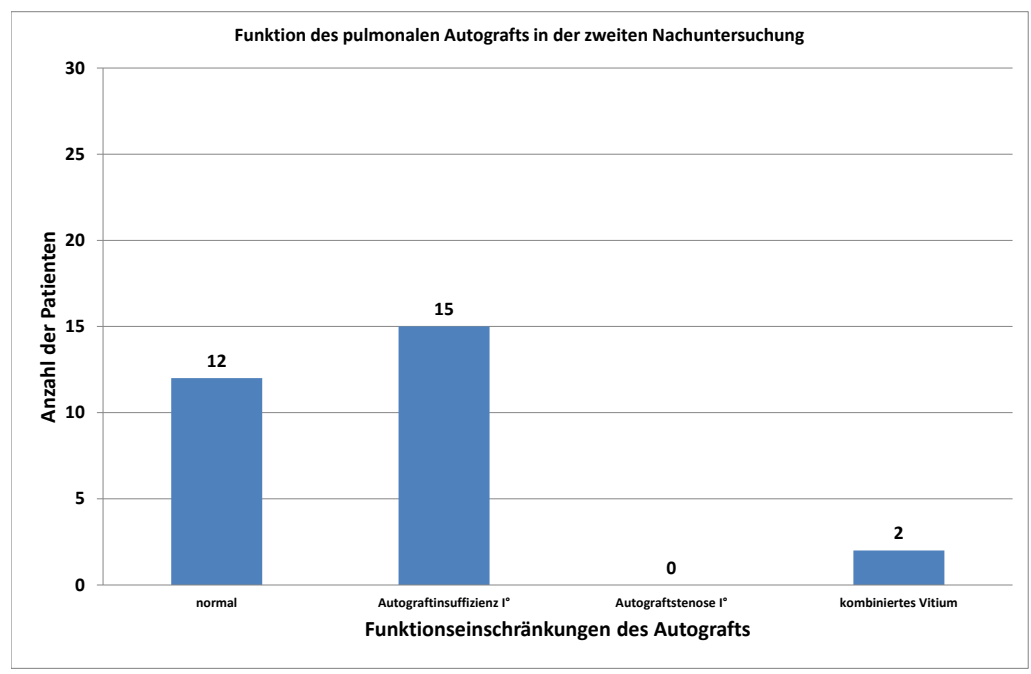

Abbildung 6: Funktion des Autografts in der zweiten post-operativen Echokardiographie $(n=29$ Patienten, da 1 Patient lost to follow-up)

\subsubsection{Regressionskurven und Z-Werte nach Daubeney et al. (1999)}

Bei den echokardiographischen Nachuntersuchungen wurden die Durchmesser des pulmonalen Autografts bestimmt. Dabei konnten 92\% der Messwerte bestimmt und ausgewertet werden. Die fehlenden Messwerte sind zum Teil auf die schlechte Qualität der Video-Aufzeichnungen der weiter zurückliegenden Untersuchungen zurückzuführen, die die exakte Messung unmöglich machte. Einige Patienten wurden in externen kinderkardiologischen Praxen nachuntersucht, so dass die Werte nicht ermittelt werden konnten.

In den Abbildungen 7.9 sind die gemessenen Aortendurchmesser für Neo-Aortenklappenring, Neo-Aortenwurzel und sino-tubulären Übergang gegen die zugehörigen BSA-Werte aufgetragen. Zusätzlich wurden in die Grafiken die von Daubeney et al. (1999) erstellten Regressionskurven eingezeichnet, die anhand der Werte gesunder Kinder ermittelt wurden.

Anhand der Grafiken kann somit ein Bezug zwischen einem Kollektiv gesunder Kinder und dem der in Göttingen mittels Ross-Operation behandelten Patienten hergestellt werden. Für jede gemessene Struktur ist jeweils eine Grafik mit den Werten der ersten und 
der zweiten echokardiographischen Nachuntersuchung dargestellt. Anhand des Abstandes jedes einzelnen Punktes von der Normkurve ist der jeweilige Z-Wert des Patienten zu ermitteln.
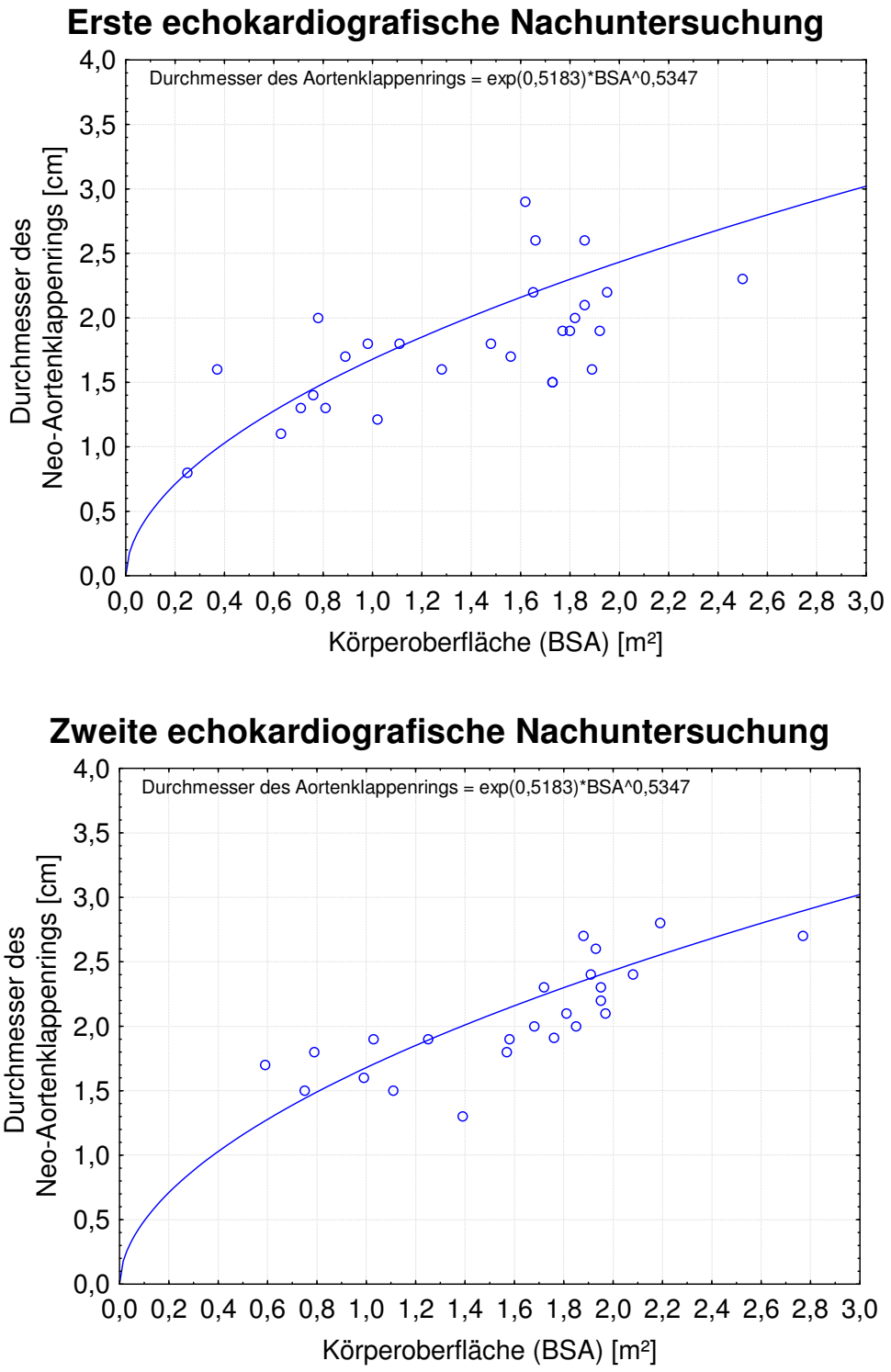

Abbildung 7: Regression nach Daubeney für den Neo-Aortenklappenring (Autograft) 

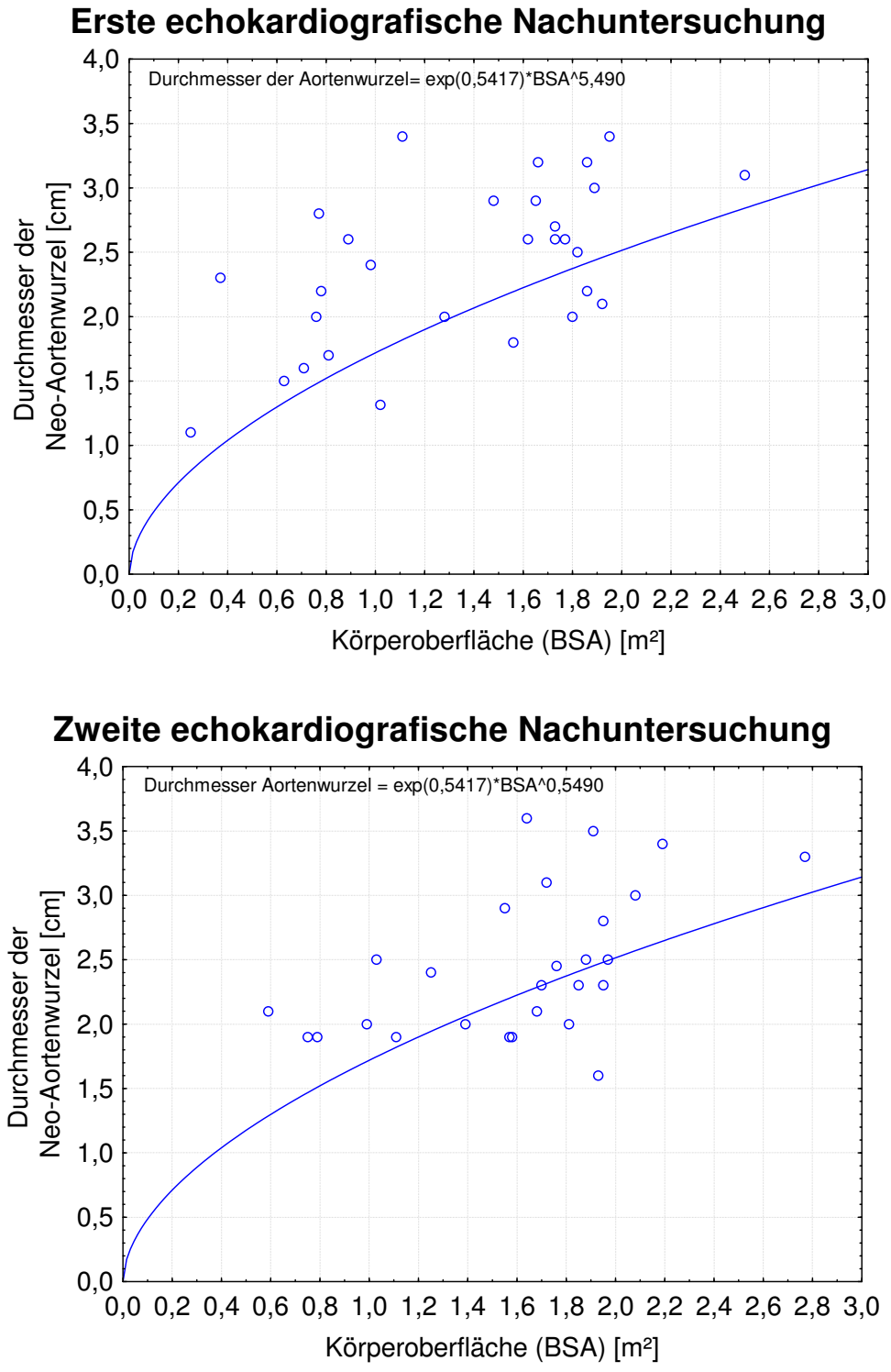

Abbildung 8: Regression nach Daubeney et al. (1999) für die Neo-Aortenwurzel (Autograft) 


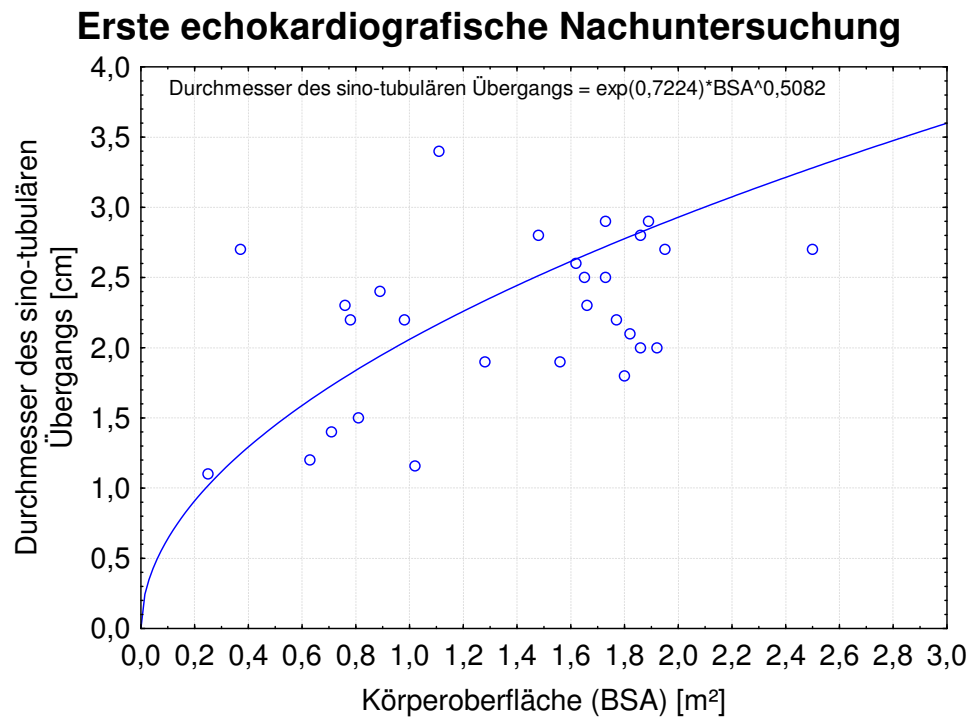

\section{Zweite echokardiografische Nachuntersuchung}

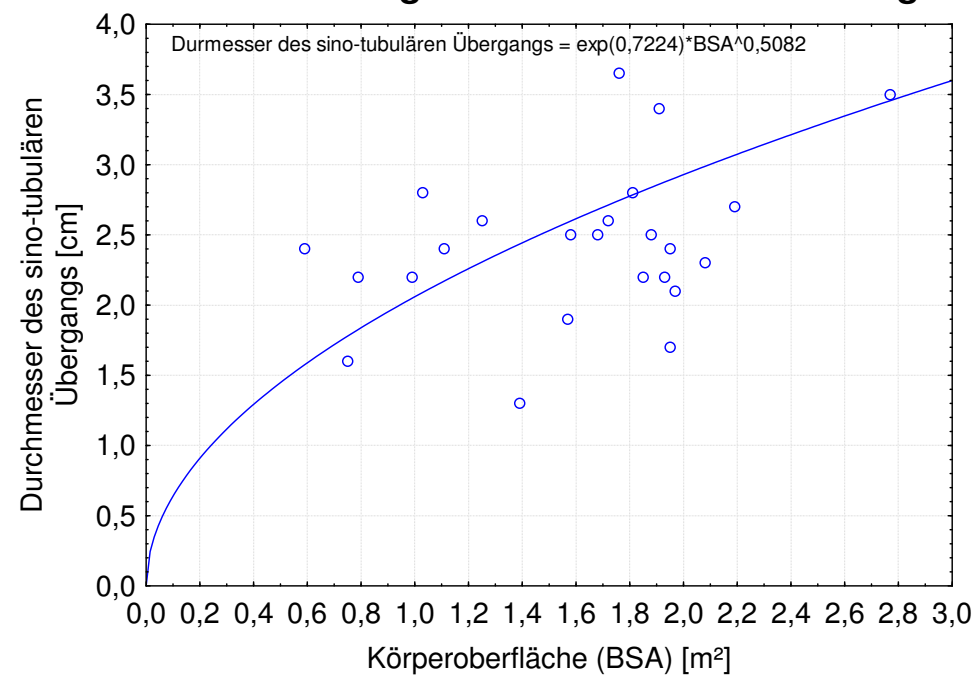

Abbildung 9: Regression nach Daubeney et al. (1999) für den sino-tubulären Übergang 
Die in Abbildung 10 dargestellten Boxplots zeigen die aus den Regressionsgraden ermittelten Z-Werte für die jeweiligen Messpunkte, also die Abweichung von der durch Daubeney et al. (1999) erstellten Regressionskurve. Die Z-Werte sind jeweils für die erste und die zweite verwendete Echokardiographie für die Strukturen Neo-Aortenklappenring, Neo-Aortenwurzel und sino-tubulären Übergang abgebildet. Anhand der Boxplots lässt sich die Entwicklung der Z-Werte zwischen erster und zweiter Echokardiographie graphisch darstellen. Für die Z-Werte der Durchmesser des Aortenklappenrings und des sino-tubulären Übergangs zeigte sich von der ersten zur zweiten echokardiographischen Messung keine signifikante Veränderung. Im Einzelnen ergab sich für den Aortenklappenring ein $\mathrm{p}$-Wert von $\mathrm{p}=0,768331$ und für den sino-tubulären Übergang ein $\mathrm{p}$-Wert von $\mathrm{p}=0,834408$. Die Zunahme der Durchmesser entsprachen dem physiologisch zu erwartendem Wachstum.

Für den Messpunkt der Aortenwurzel zeigte sich ein signifikant abnehmender Z-Wert, $\mathrm{p}=0,033045$. Demnach haben sich im Langzeitverlauf die Messwerte der Durchmesser der Aortenwurzel signifikant an die Regressionskurve angeglichen.

Es ist folglich im beobachteten Zeitraum zu keiner Dilatation der Aortenwurzel gekommen. Vielmehr hat der Aortenwurzeldurchmesser weniger stark zugenommen, als es im Rahmen des Körperwachstums zu erwarten gewesen wäre sich somit an das gesunde Kollektiv angenähert. 


\section{Boxplot für die Z-Werte des Aortenklappenrings}

gruppiert nach Nachuntersuchungszeitpunkt

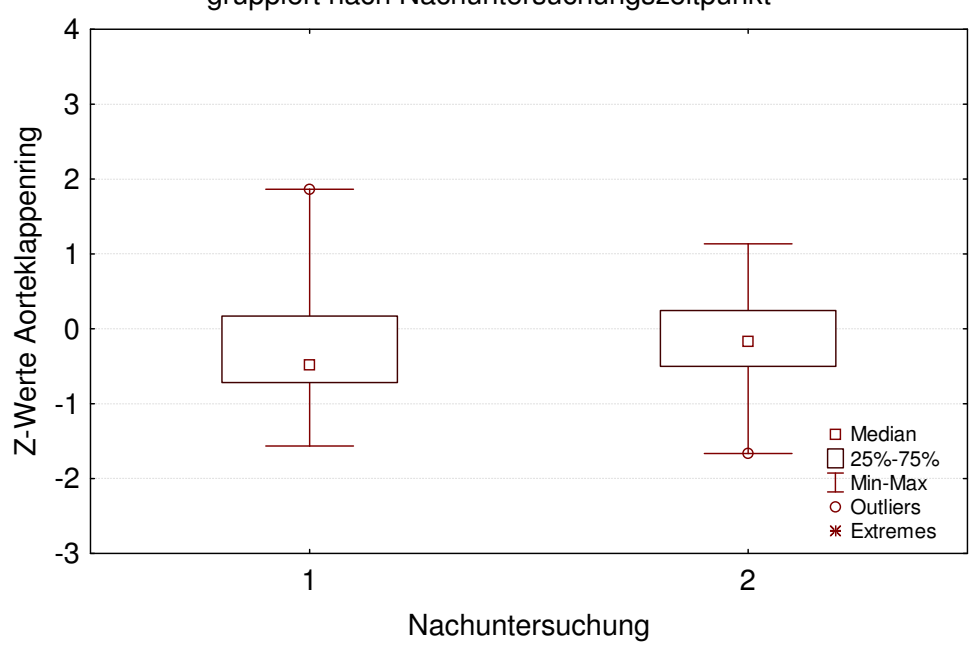

Boxplot für die Z-Werte der Aortenwurzel

gruppiert nach Nachuntersuchungszeitpunkt

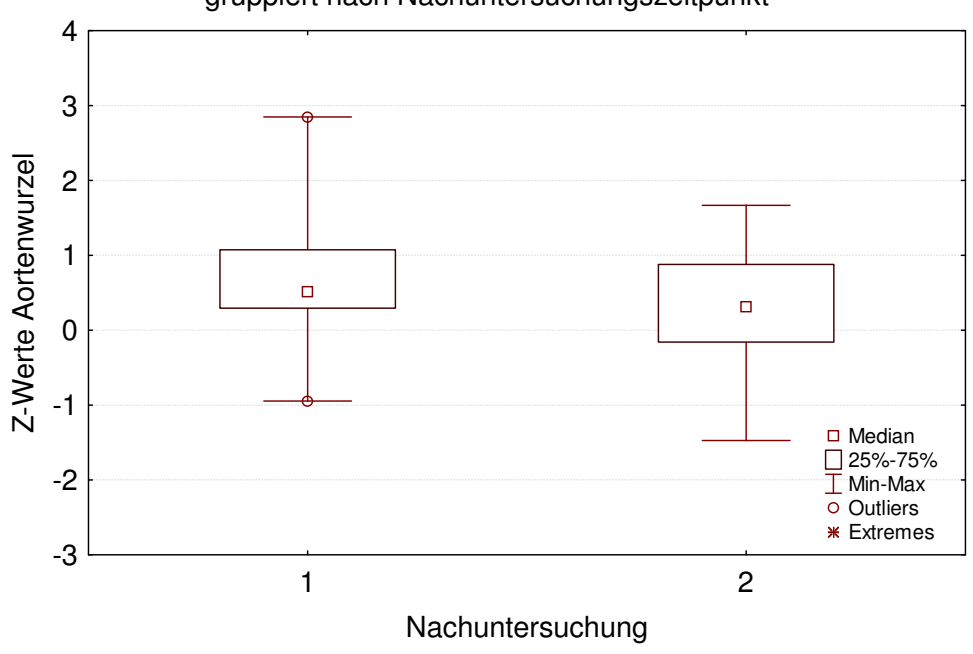

Boxplot für die Z-Werte des sino-tubulären Übergangs

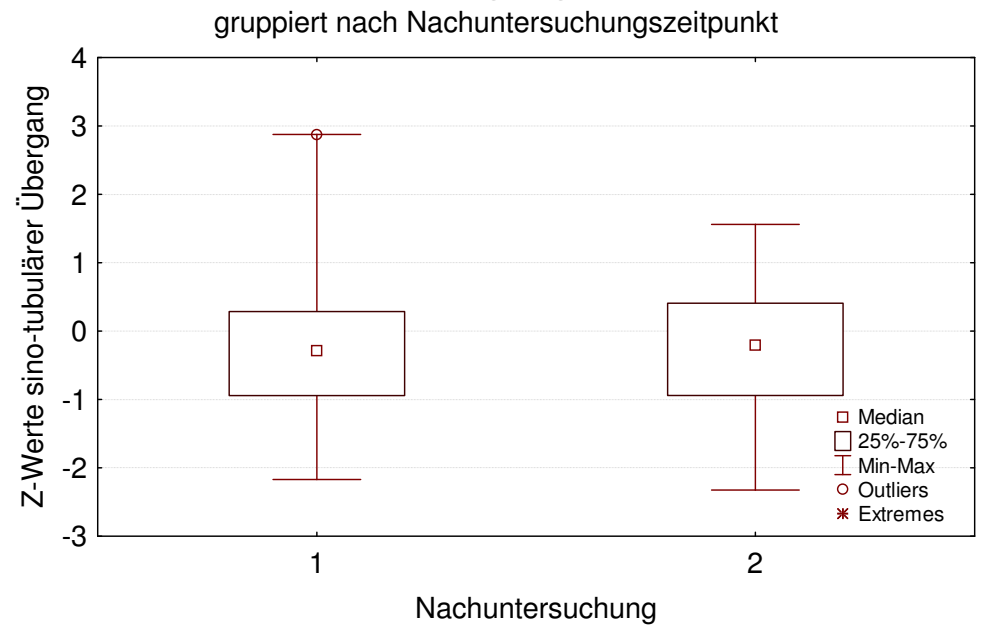

Abbildung 10: Z-Werte für die Durchmesser von Neo-Aortenklappenring, NeoAortenwurzel und sino-tubulärem Übergang nach Daubeney et al. (1999) 


\subsubsection{Regressionskurven und Z-Werte nach Pettersen et al. (2008)}

Abgebildet sind analog zu Daubeney et al. (1999) die durch Pettersen et al. (2008) errechneten Regressionskurven für die Durchmesser des Aortenklappenrings, des sinotubulären Übergangs und der Aortenwurzel normaler Kinder und Jugendlicher im Verhältnis zur Körperoberfläche. Die bei den Göttinger Patienten gemessenen Werte für die jeweiligen Durchmesser und Körperoberflächen sind als Punkte in die Grafiken eingetragen (Abbildung 11,13). Wieder lassen sich die Z-Werte anhand des Abstandes jedes einzelnen Punktes von der Normkurve errechnen.

Der stark unterschiedliche Verlauf der Kurven zwischen Daubeney et al. (1999) und Pettersen et al. (2008) ist auf die zugrundeliegenden Regressionsmodelle zurückzuführen. 

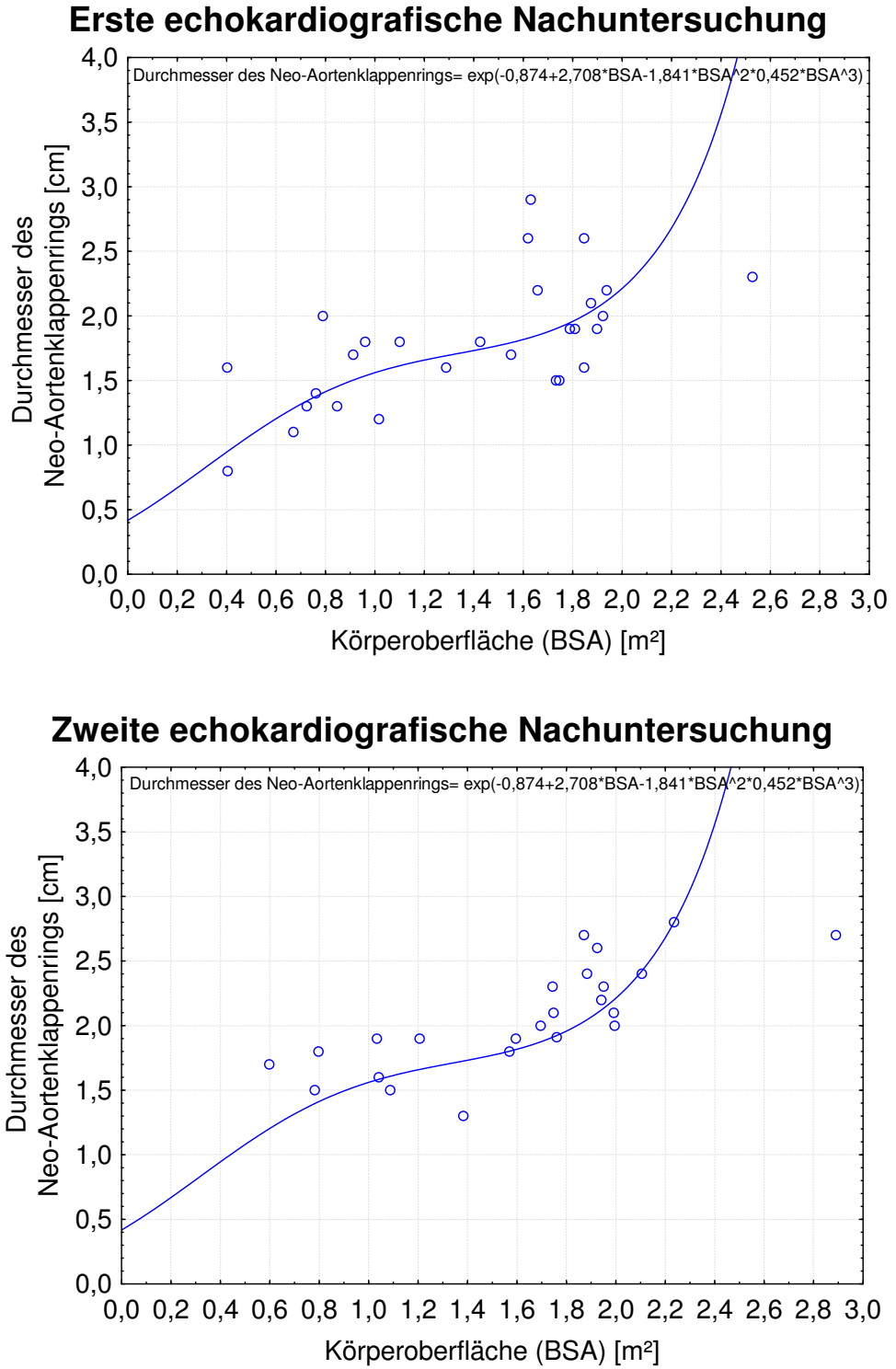

Abbildung 11: Regression nach Pettersen et al. (2008) für den Neo-Aortenklappenring 

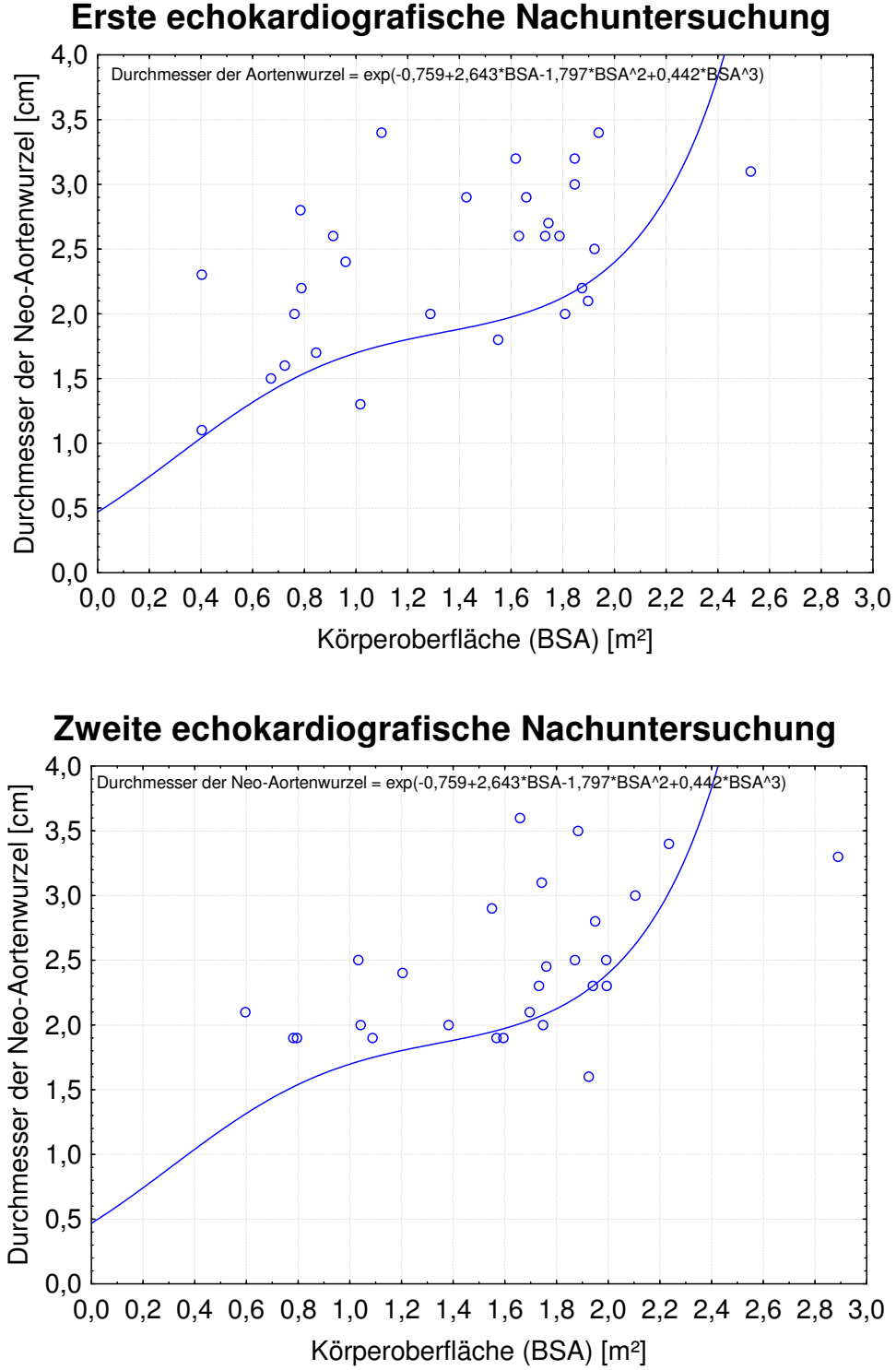

Abbildung 12: Regression nach Pettersen et al. (2008) für die Neo-Aortenwurzel 

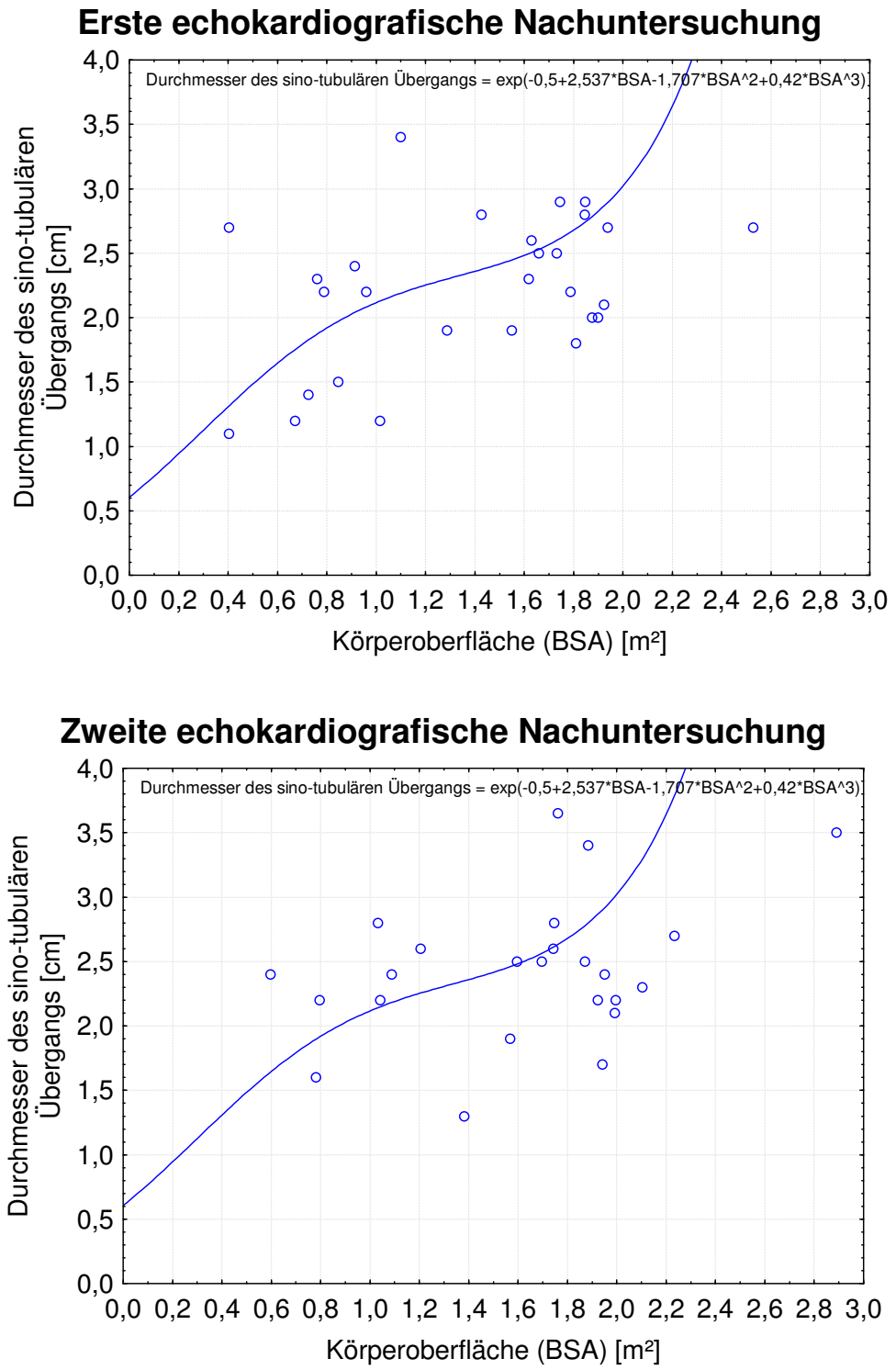

Abbildung 13: Regression nach Pettersen et al. (2008) für den sino-tubulären Übergang 
Die für die erste und zweite Nachuntersuchung ermittelten Z-Werte sind als Boxplots dargestellt (Abbildung14). Mittels t-Test für verbundene Stichproben wurde analog zur Auswertung nach Daubeney et al. (1999) untersucht, ob es zu einer signifikanten Veränderung der Z-Werte zwischen der ersten und zweiten Nachuntersuchung gekommen war. Es ergaben sich für die Durchmesser des Neo-Aortenklappenrings $(p=0,765057)$ und des sino-tubulären Übergangs $(\mathrm{p}=0,505552)$ keine signifikanten Veränderungen der Z-Werte zwischen der ersten und der letzten Echokardiographie.

Analog zu der Untersuchung der Z-Werte nach Daubeney et al. (1999) zeigte sich auch für die Z-Werte nach Pettersen et al. (2008) eine signifikante Veränderung der Z-Werte der Neo-Aortenwurzel $(\mathrm{p}=0,044210)$ in Form einer Annährung an die Normkurve.

Somit lässt sich nach diesem Modell ebenfalls die Annäherung der gemessenen Neo-Aortenwurzel-Durchmesser an ein Normalkollektiv nachweisen. Aufgrund der untersuchten Daten lässt sich keine Evidenz für die Dilatation des Autografts nachweisen. 


\section{Boxplot für die Z-Werte des Aortenklappenrings}

gruppiert nach Nachuntersuchungszeitpunkt

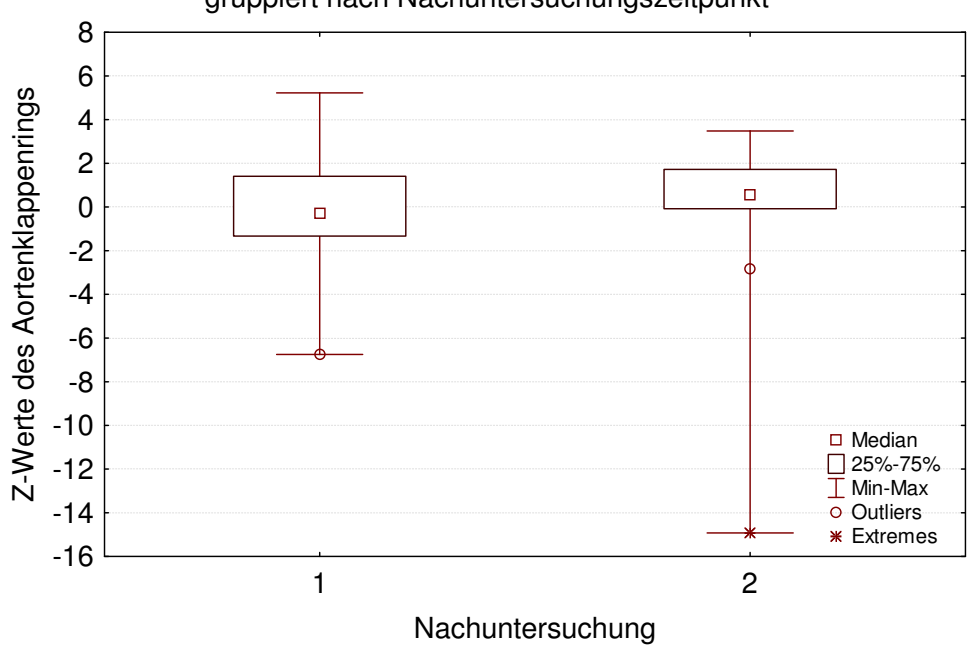

Boxplot für die Z-Werte der Aortenwurzel

gruppiert nach Nachuntersuchungszeitpunkt

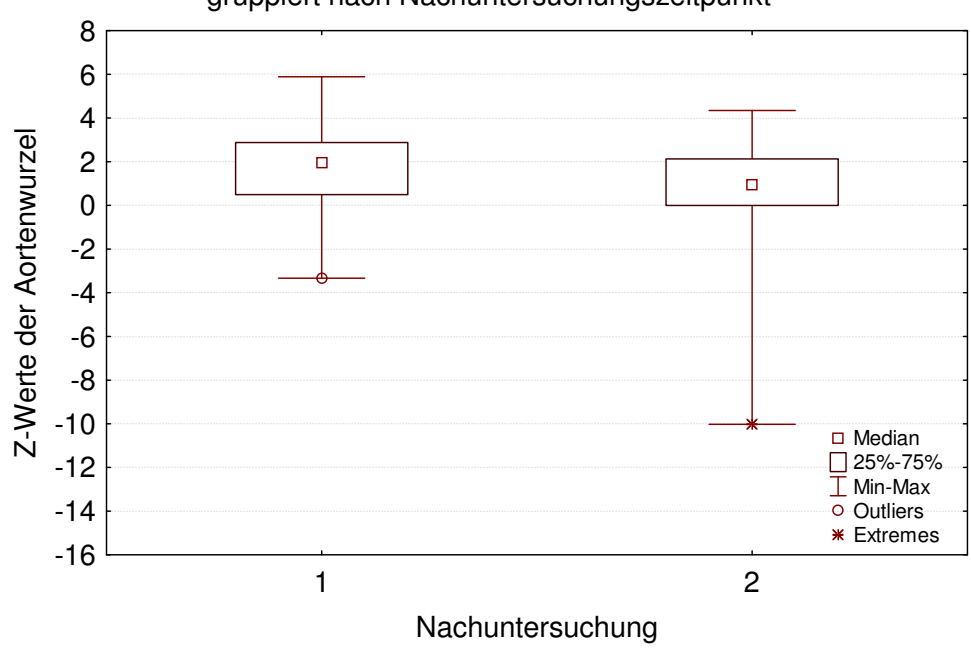

Boxplot für die Z-Werte des sino-tubulären Übergangs

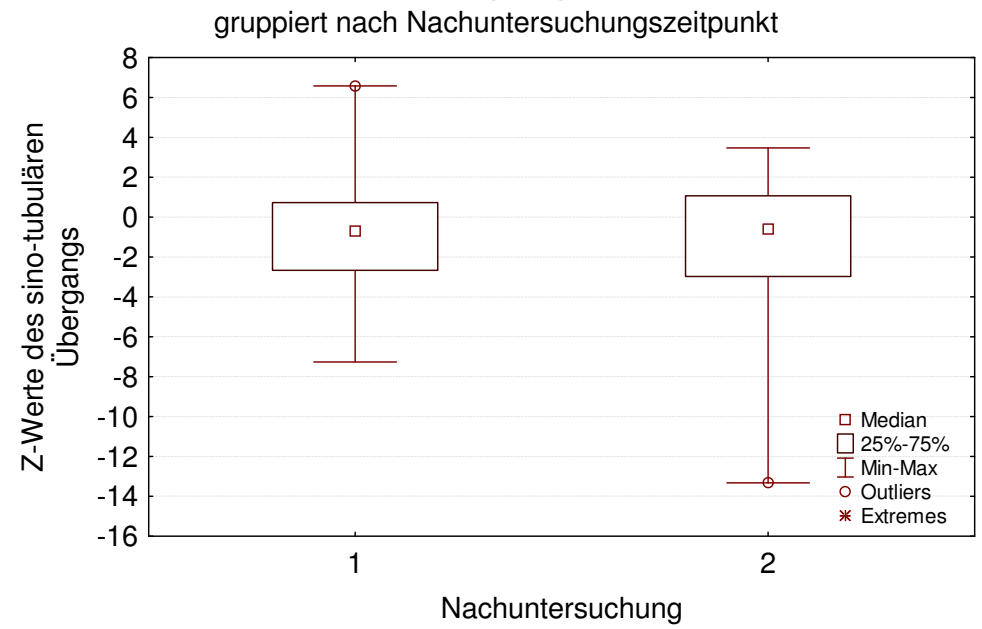

Abbildung 14: Z-Werte für die Durchmesser von Neo-Aortenklappenring, NeoAortenwurzel und sino-tubulärem Übergang nach Pettersen et al. (2008) 


\subsection{Die Ergebnisse der echokardiographischen Nachuntersuchungen des linken Ventrikels}

Die Arbeitsgruppe von Pettersen et al. (2008) hat in ihren echokardiographischen Untersuchungen gesunder Kinderherzen auch bestimmte Durchmesser des linken Ventrikels berücksichtigt, die standardmäßig bei Echokardiographien bestimmt werden. Für diese wurden ebenfalls Regressionskurven erstellt.

In den verwendeten echokardiographischen Nachuntersuchungen konnten insgesamt 95\% der Messungen durchgeführt, dokumentiert und ausgewertet werden. Die fehlenden Werte sind wiederum durch die teils schlechte Qualität der Aufnahmen oder fehlende Dokumentation des Untersuchers zu erklären.

Nachfolgend dargestellt sind die Regressionskurven nach Pettersen et al. (2008), von denen sich die vorhergesagten Werte für den jeweils untersuchten Durchmesser im Verhältnis zur Körperoberfläche ablesen lassen. Als Punkte in die Grafik sind die bei den in Göttingen operierten Kindern gemessenen Durchmesser und Körperoberflächen eingetragen (Abbildungen 16,18). 

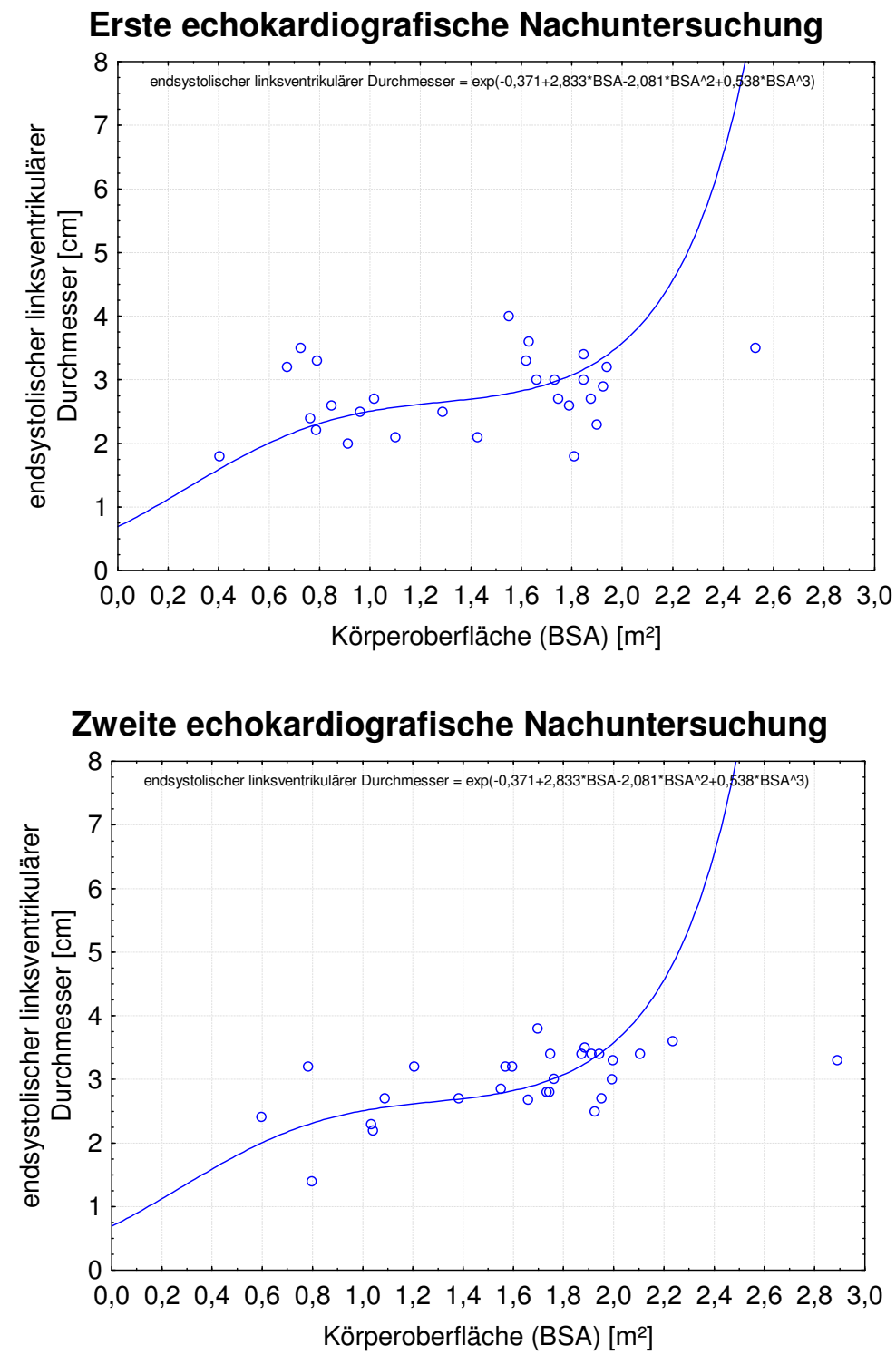

Abbildung 15: Regression nach Pettersen et al. (2008) für den LVESD 

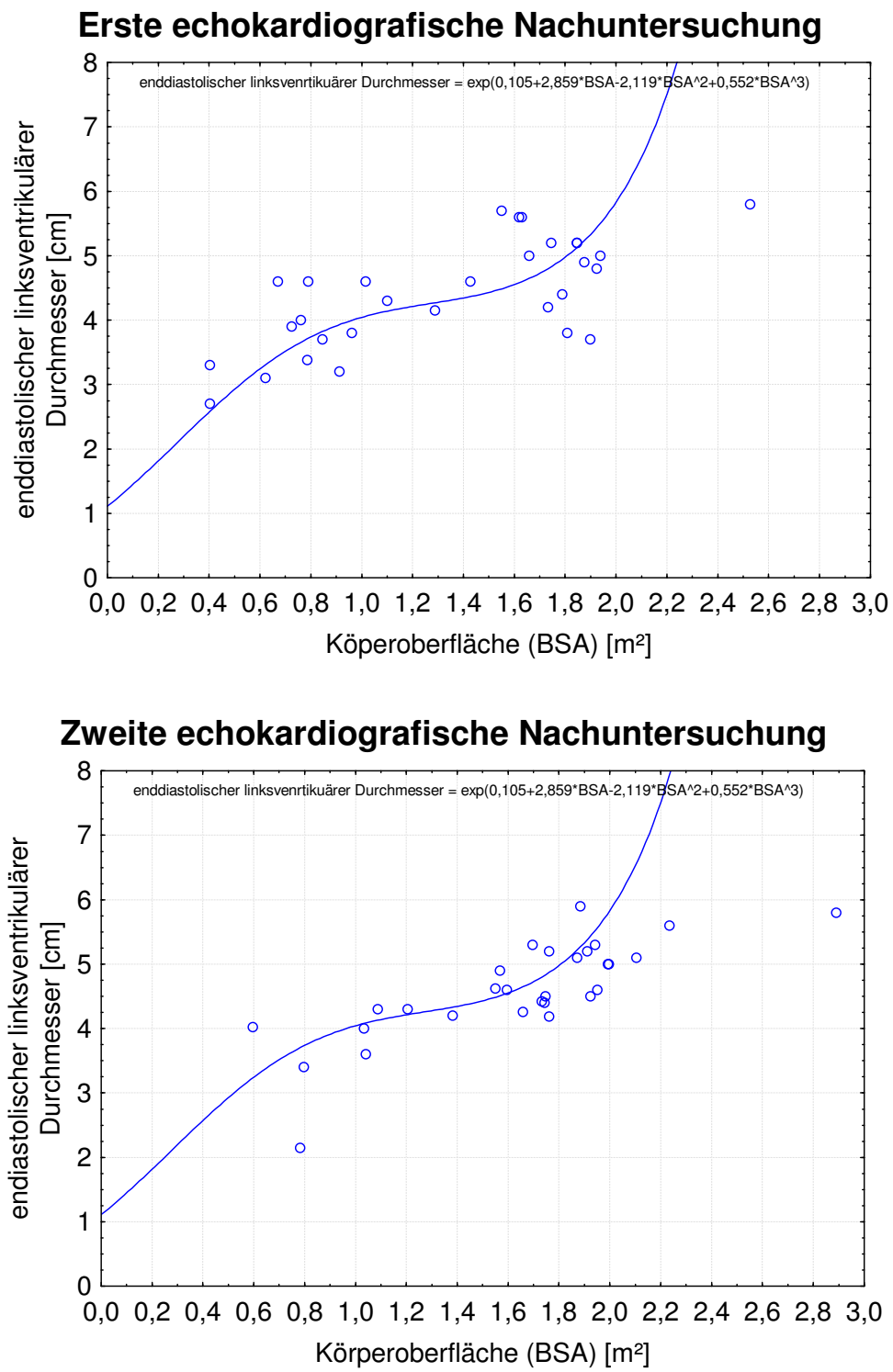

Abbildung 16: Regression nach Pettersen et al. (2008) für den LVEDD 

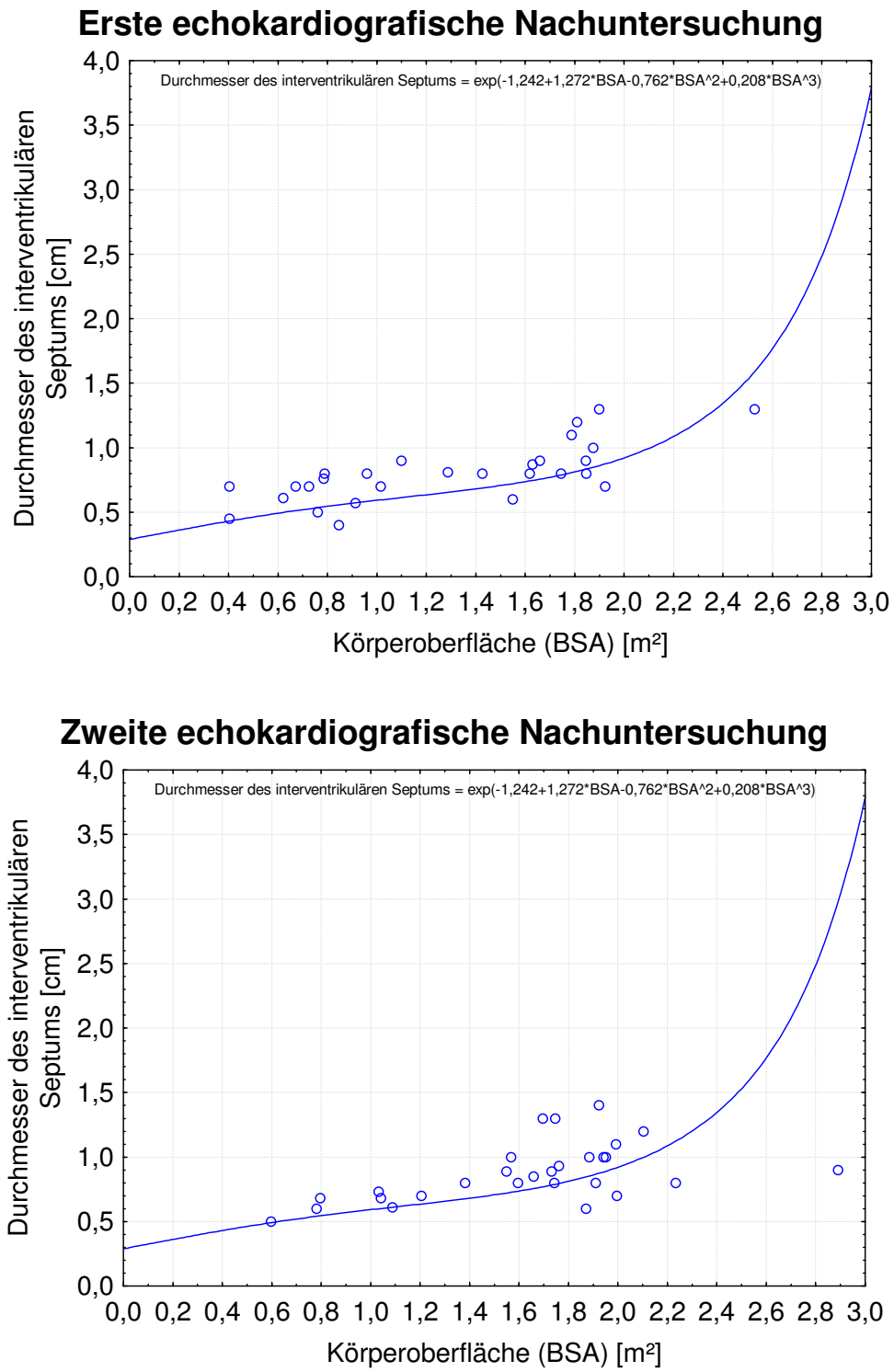

Abbildung 17: Regression nach Pettersen et al. (2008) für den Durchmesser des IVS 

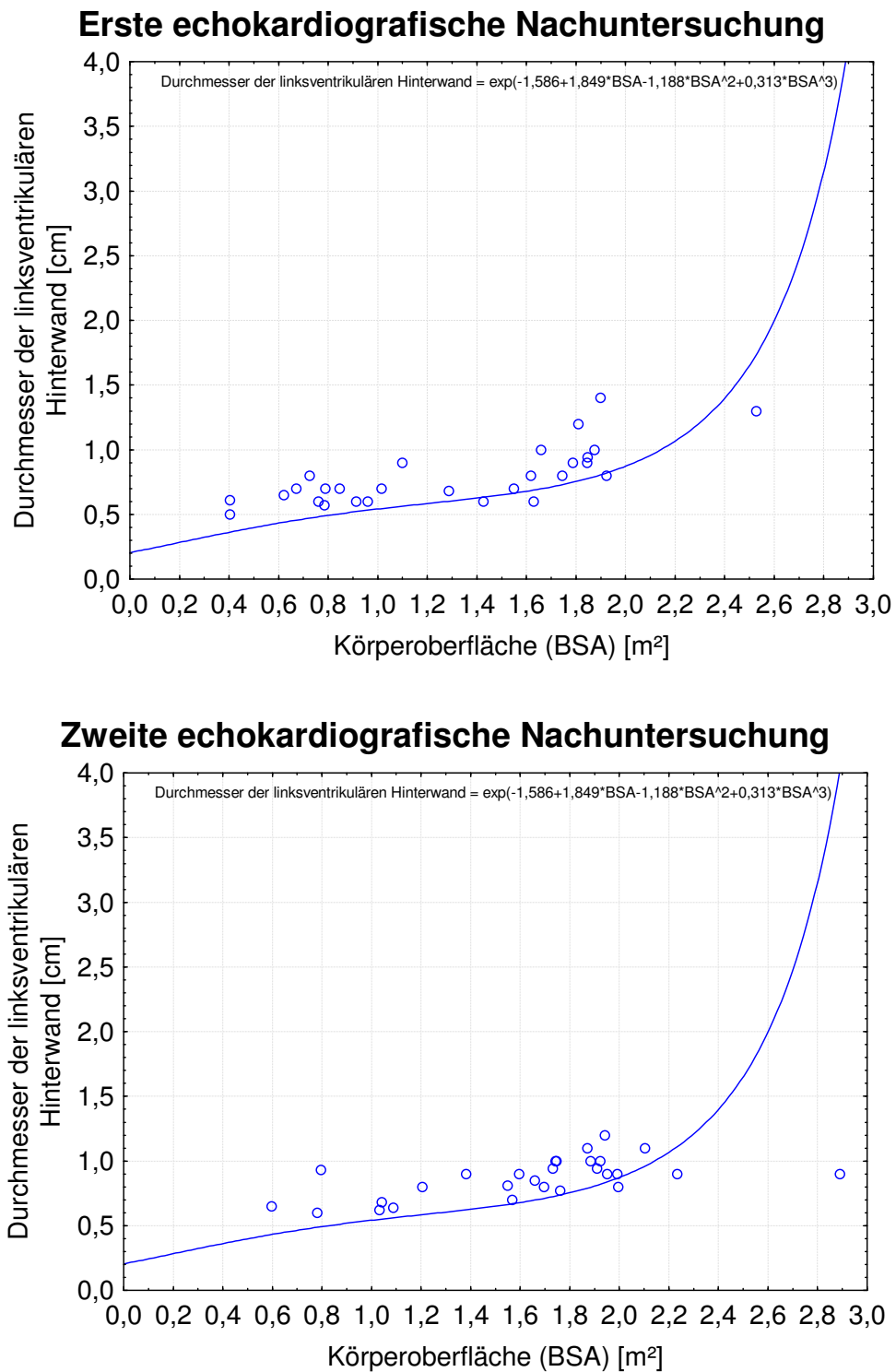

Abbildung 18: Regression nach Pettersen et al. (2008) für den Durchmesser der linksventrikulären HW 
Die nach Pettersen et al. (2008) ermittelten Z-Werte für die Durchmesser des linken Ventrikels sind in Form von Boxplots dargestellt (Abbildung 19 20). In einer Grafik sind jeweils die ermittelten Z-Werte zum ersten und zum zweiten Untersuchungszeitpunkt gegenübergestellt. Durch den t-Test für verbundene Stichproben wurde auch für die linksventrikulären Durchmesser überprüft, ob es zu signifikanten Veränderungen gekommen war.

\section{Boxplot für die Z-Werte des linksventrikulären endsystolischen Durchmessers}

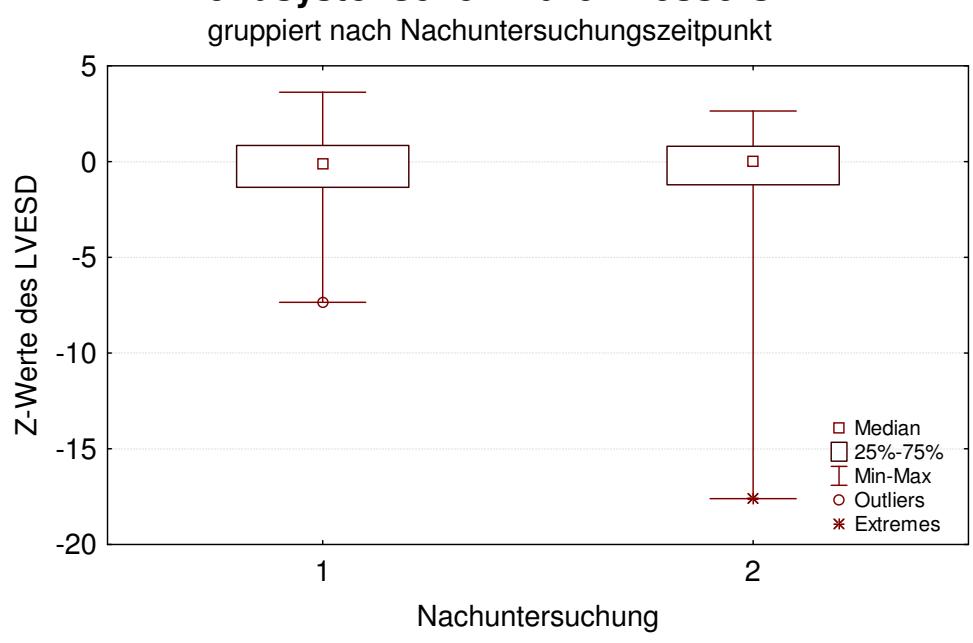

\section{Boxplot für die Z-Werte des linksventrikulären enddiastolischen Durchmessers}

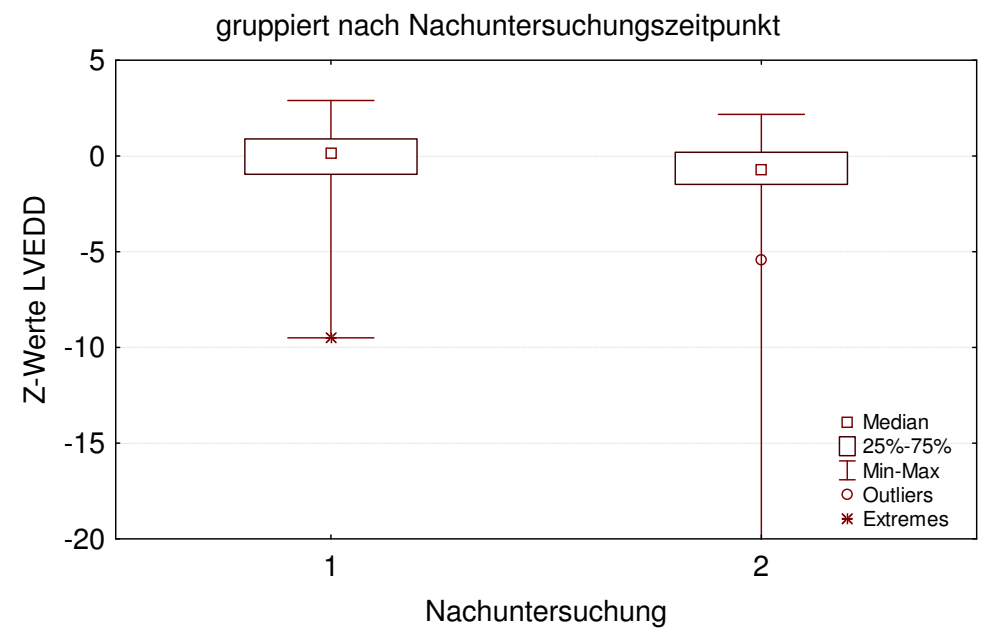

Abbildung 19: Z-Werte für LVESD und LVEDD nach Pettersen et al. (2008)

Für die LVESD ( $p=0,219500)$, den Durchmesser des IVS $(p=0,397496)$ und den Durchmesser der HW des linken Ventrikels $(p=0,285809)$ traten zwischen der ersten und zweiten echokardiographischen Messung keine signifikanten Veränderungen der Z-Werte auf. 
Boxplot für die Z-Werte des interventrikulären

Septums

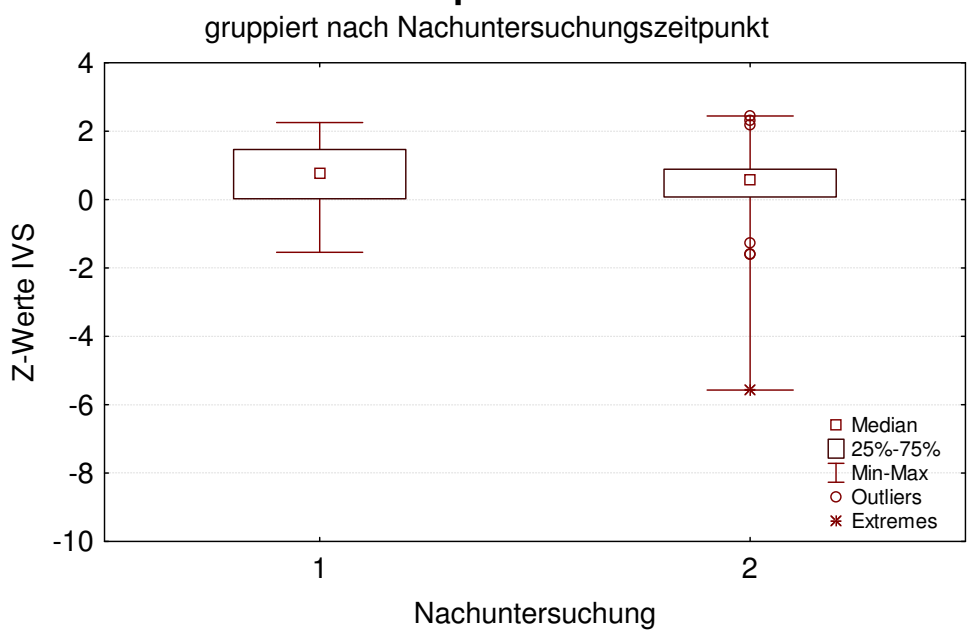

Boxplot für die Z-Werte der linksventrikulären

Hinterwand

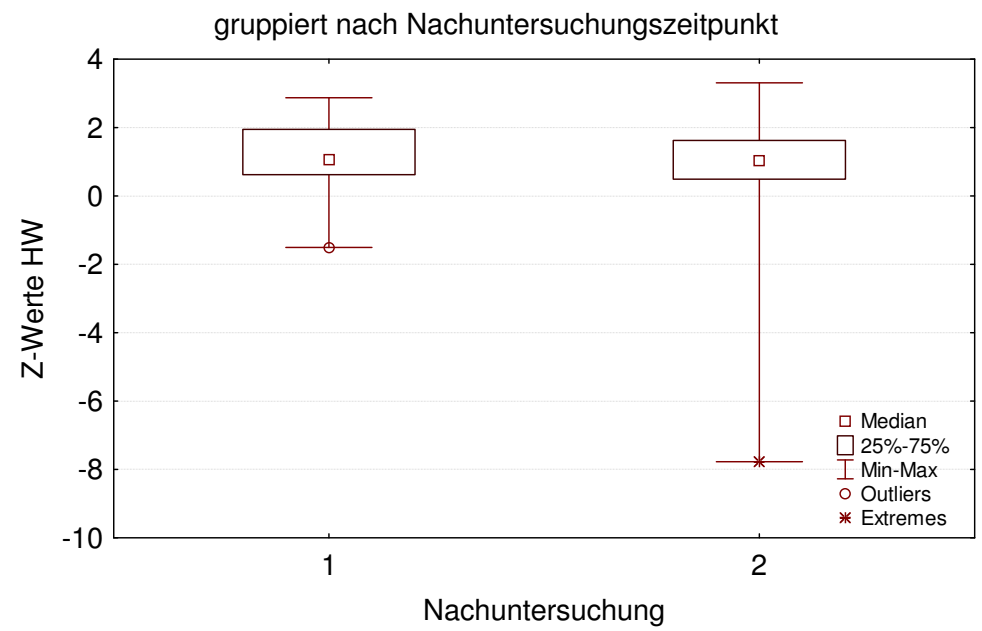

Abbildung 20: Z-Werte für IVS und HW nach Pettersen et al. (2008)

Für den LVEDD ließ sich eine signifikante Abnahme der Z-Werte feststellen $(p=0,019325)$.

Es kam zu keiner Hypertrophie des linken Ventrikels im postoperativen Verlauf. 


\subsection{Die Langzeitentwicklung der Homografts und Xenografts}

In der ersten formal verwertbaren postoperativen echokardiographischen Nachuntersuchung zeigte sich bei 7 Patienten eine Insuffizienz des Homografts. Alle Insuffizienzen waren ohne klinische Bedeutung (trivial). Bei 3 Patienten trat eine Stenosierung des Homografts auf. Davon war eine Stenose mittelgradig (jet-Geschwindigkeit 3,8 m/s). Bei 3 Patienten bestand eine Kombination aus beiden Störungen ohne hämodynamische Relevanz. Bei 1 Patienten war zu diesem Zeitpunkt schon ein Austausch des Homografts erfolgt (siehe Abbildung 21).

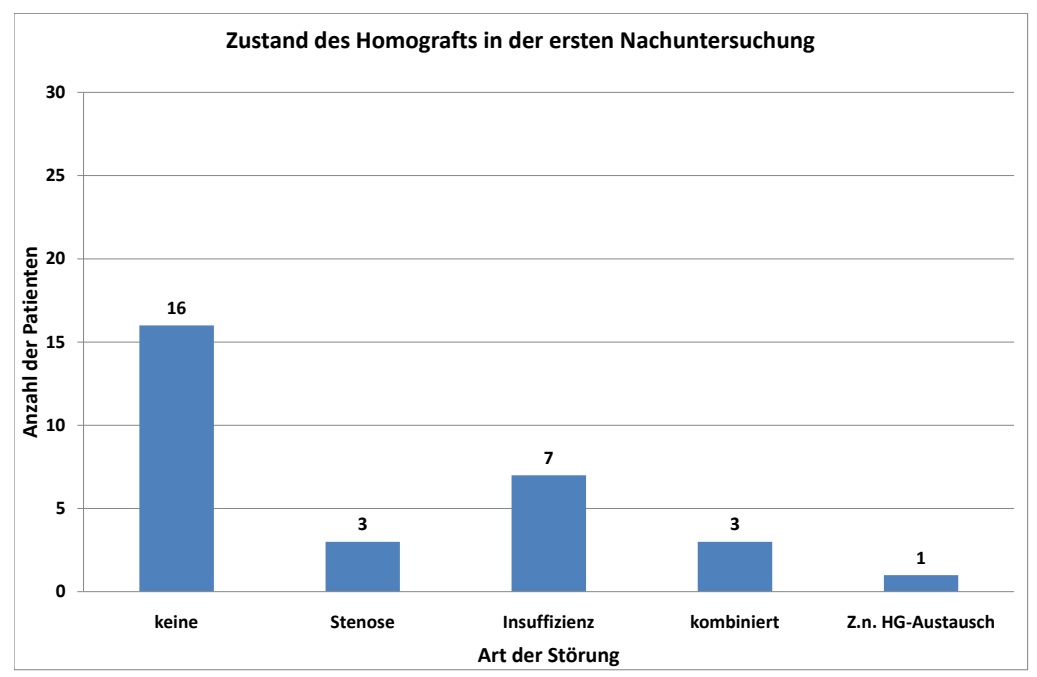

Abbildung 21: Zustand des Homografts/Xenografts in der ersten post-operativen Echokardiographie ( $\mathrm{n}=30$ Patienten, da 1 Patientin verstorben) 


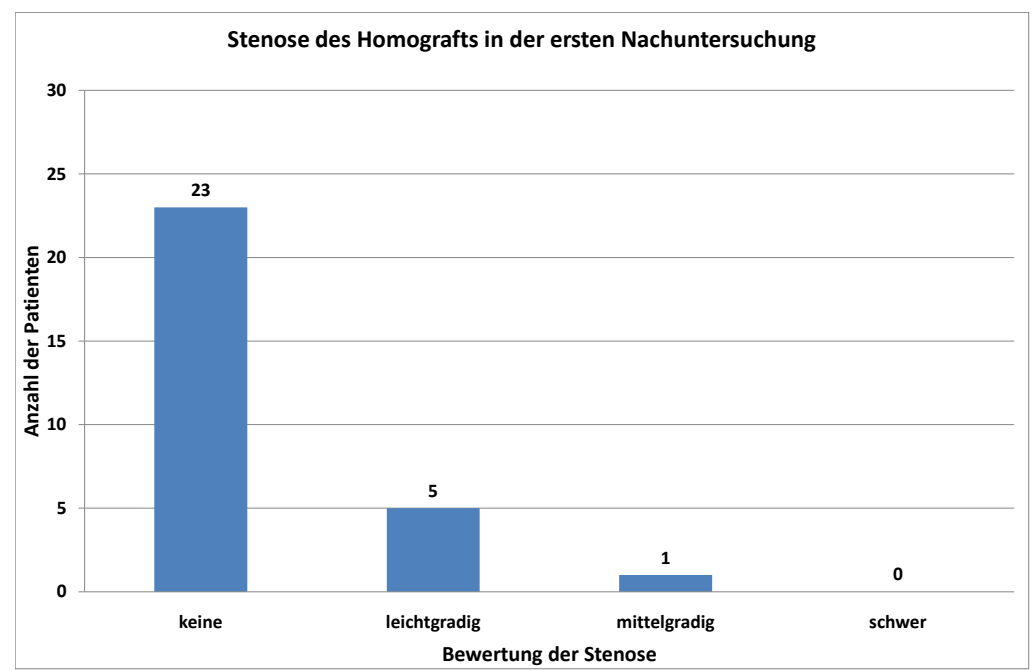

Abbildung 22: Homograft-/Xenograftstenose in der ersten post-operativen Echokardiographie $(\mathrm{n}=29)$

*Patient mit bereits ausgetauschtem Homograft wurde nicht aufgeführt, 1 Patient lost to follow-up

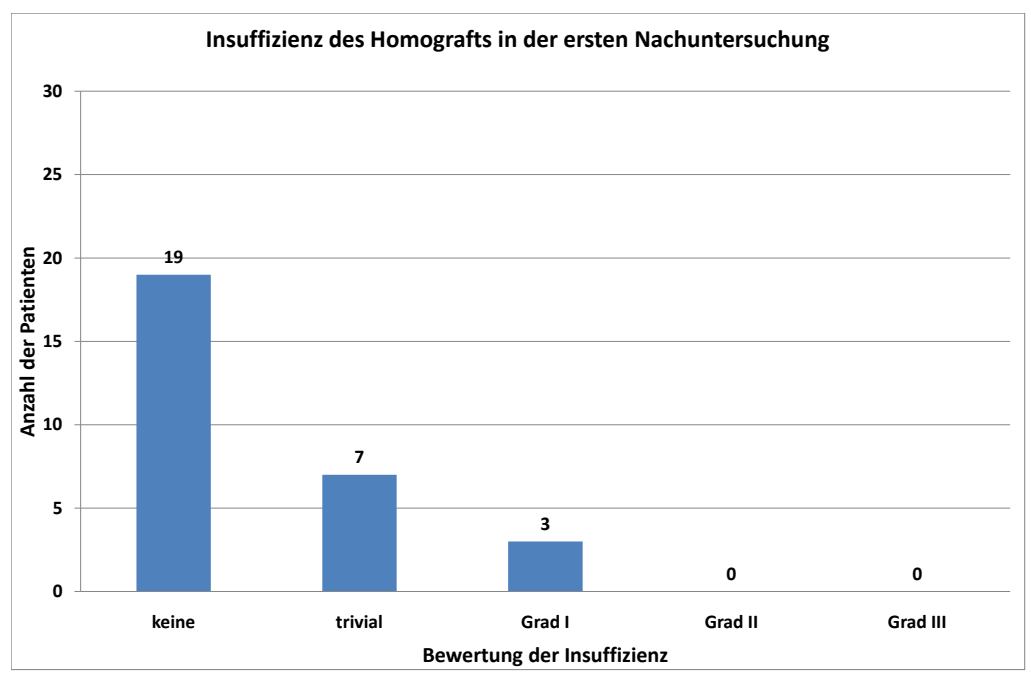

Abbildung 23: Homograft-/Xenograftinsuffizienz in der ersten post-operativen Echokardiographie $(\mathrm{n}=29)$

*Patient mit bereits ausgetauschtem Homograft wurde nicht aufgeführt, 1 Patient lost to follow-up 
In der zweiten echokardiographischen Nachuntersuchung zeigten 3 Patienten eine Insuffizienz des Homografts/Xenografts, bei 6 Patienten konnte eine Homograft-/Xenograftstenose festgestellt werden und 10 Patienten wiesen eine kombinierte Störung auf. Die Funktion der Homografts/Xenografts hatte sich insgesamt verschlechtert. Von diesen nachgewiesen Funktionseinschränkungen war eine Stenose eines Xenografts (Contegra ${ }^{\circledR}$ ) mit einer Jet-Geschwindigkeit von $3,1 \mathrm{~m} / \mathrm{s}(38 \mathrm{mmHg})$ klinisch relevant. In der grafischen Darstellung zur Funktion des Homografts/Xenografts sind die Patienten, die zwischenzeitlich bereits einen Homograft-Austausch erhalten hatten, nicht mit aufgeführt (Abbildung 24.

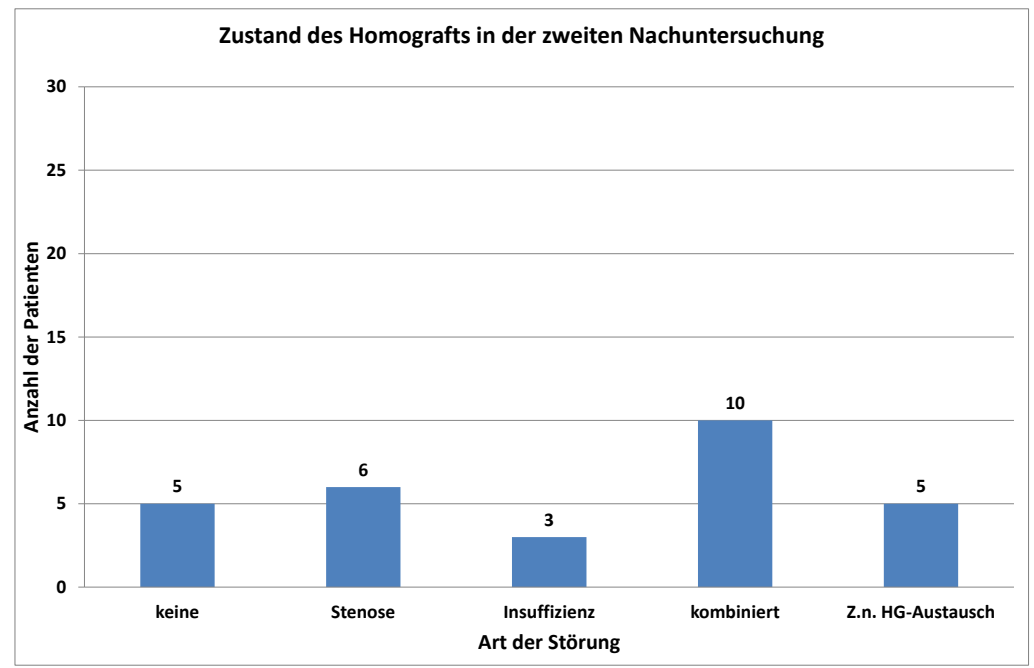

Abbildung 24: Zustand des Homografts/Xenografts in der zweiten post-operativen Echokardiographie $(\mathrm{n}=29)$

Im postoperativen Verlauf benötigten insgesamt 5 Patienten einen Austausch des Homografts. Bei den ausgetauschten Conduits handelte es sich um 3 Pulmonalishomografts und 2 Aortenhomografts. Von den verwendeten Xenografts musste im Nachbeobachteten Zeitraum keine ausgetauscht werden. 


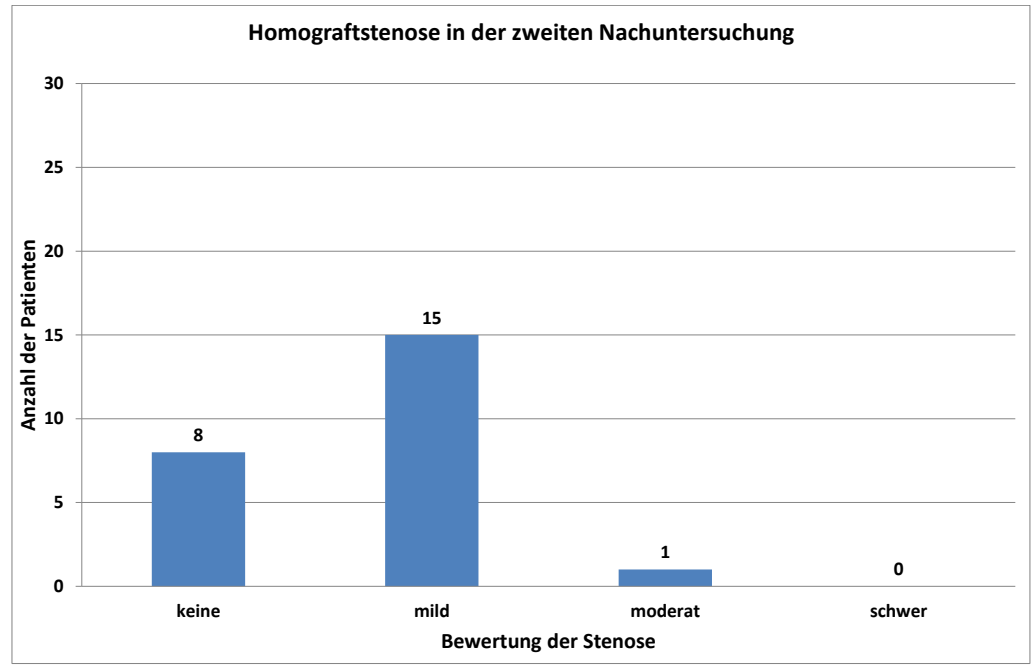

Abbildung 25: Homograft-/Xenograftstenose in der zweiten post-operativen Echokardiographie $(\mathrm{n}=24)$

*Patienten mit bereits ausgetauschten Homografts wurden nicht aufgeführt, 1 Patient lost to follow-up

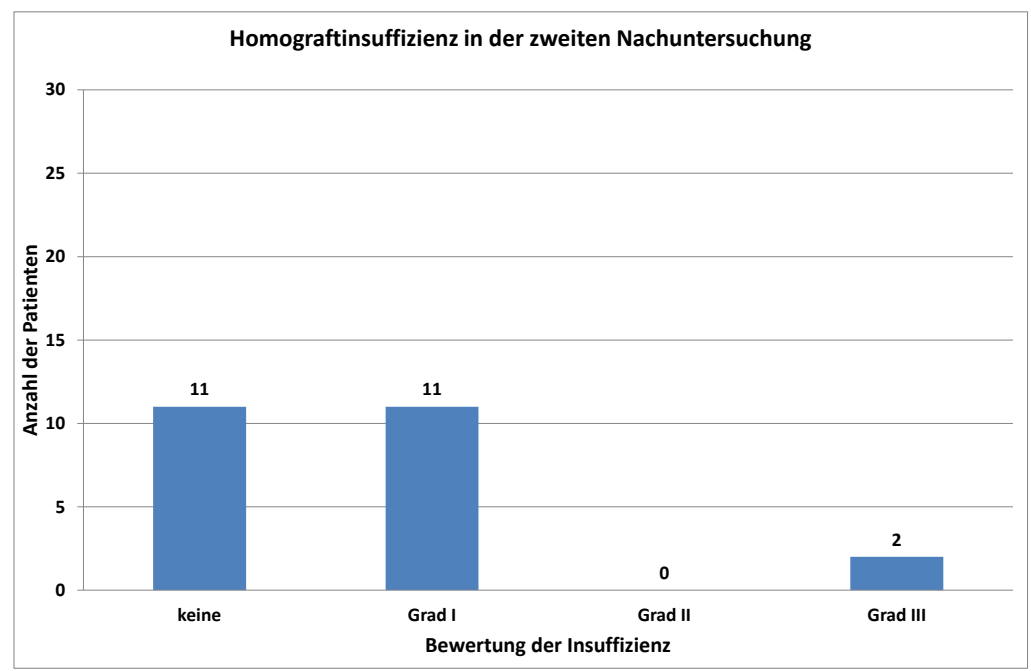

Abbildung 26: Homograft-/Xenograftinsuffizienz in der zweiten post-operativen Echokardiographie $(\mathrm{n}=24)$

*Patienten mit bereits ausgetauschten Homografts wurden nicht aufgeführt, 1 Patient lost to follow-up 


\section{Diskussion}

In der Abwägung des Für und Wider der Ross-Operation steht die Frage im Vordergrund, ob die Vorteile der nicht notwendigen Antikoagulation, des Mitwachsens und der Regenerationsfähigkeit der autologen Klappe und die gute Funktion des pulmonalen Autografts das Risiko, eine Einherzklappenerkrankung in eine potentielle Zweiherzklappenerkrankung umzuwandeln, rechtfertigen. Die begrenzte Haltbarkeit des Pulmonalis-Homografts als Neo-Pulmonalklappe ist nachgewiesen. Die Entwicklung des Autografts ist weiter unter kritischer Beobachtung. Einige Arbeitsgruppen beschreiben eine übermäßige Dilatation, während andere ein adäquates Mitwachsen der Klappe in ihrer neuen Position beobachten. Ich werde im Folgenden die Ergebnisse der Göttinger Patienten im Kontext mit den in den letzten Jahren veröffentlichten Ergebnissen anderer Studien im Hinblick auf die Morbidität und Letalität, die Entwicklung des Autografts, die Entwicklung des linken Ventrikels und die Haltbarkeit des Homografts bzw. Xenografts in Pulmonalklappenposition diskutieren.

\subsection{Morbidität und Letalität}

Die Ross-Operation ist unter der Voraussetzung, dass nicht bereits schwerwiegende Herzschäden oder zusätzliche Fehlbildungen vorliegen, die ein erhöhtes Operationsrisiko bedingen, mit geringer Morbidität und Letalität durchführbar. Als Risikofaktoren erscheinen ein sehr junges Operationsalter, komplexe linksventrikuläre Pathologien mit bereits entstandenen strukturellen Herzschäden und die Notfallindikation z.B. bei florider Endokarditis.

Williams et al. veröffentlichten 2005 die Ergebnisse von 27 Ross-Patienten im Alter von unter 18 Monaten, die zwischen 1991 und 2004 operiert worden waren. 1 Patient hatte postoperativ neurologische Symptome gezeigt, die im Langzeitverlauf rückläufig waren. Bei 3 Patienten war postoperativ ein AV-Block III. Grades aufgetreten, der sich bei 1 Patienten zurückbildete und bei 1 weiteren Patienten die Implantation eines Herzschrittmachers erforderte. Der 3. Patient starb im postoperativen Verlauf an bilateralen Pneumothoraces. Bei 1 anderen Patienten trat nur vorübergehend ein AV-Block II. Grades auf. Innerhalb des Krankenhausaufenthaltes starben 3 Patienten. Spättodesfälle traten während des beschriebenen Nachuntersuchungszeitraums nicht auf (Williams et al. 2005).

Stewart et al. berichteten 2007 von 46 Kindern- und Jugendlichen, bei denen eine Ross- 
bzw. Ross-Konno-Operation (11 Patienten) vorgenommen worden war und die über 11 Jahre nachuntersucht wurden (1994-2005). In 6 Fällen war es perioperativ zu Komplikationen gekommen. Darunter in 1 Fall zu einer Verletzung des N. phrenicus, in 1 Fall zu einer Herzschrittmacherimplatation aufgrund eines AV-Block III. Grades und in 1 Fall zu einer Nachblutung mit anschließend notwendiger Re-Thorakotomie. Bei 2 Patienten musste bereits am 8. postoperativen Tag eine Explantation des pulmonalen Autograft wegen starker AI nach technischen Schwierigkeiten bei der Operation erfolgen. Weder früh-postoperativ, noch im späteren Verlauf kam es zu Todesfällen. Die große Zahl an Komplikationen in Bezug auf die geringe Patientenanzahl zeigt jedoch die Komplexität des Eingriffs und des intensivmedizinischen Aufwandes sowie die damit verbundenen hohen Anforderungen an Operateur und Klinik (Stewart et al. 2007).

In dem ebenfalls 2007 von Pasquali et al. vorgestellten Patientenkollektiv trat eine 30Tages-Letalität von 2,5\% (3/121) der Operierten auf. Bei diesen Patienten war es zu Herrhythmusstörungen bzw. Linksherzversagen gekommen. Bei ihnen hatten bereits vor dem Eingriff strukturelle Herzschäden aufgrund der komplexen kardialen Pathologie vorgelegen. Im weiteren Verlauf war es zu 2 späten Todesfällen aufgrund kardialer Ursachen gekommen. Diese Ergebnisse zeigten, dass die Ross-Operation auch bei Kindern mit komplexer linksventrikulärer Fehlbildung mit vergleichsweise geringer Letalität durchführbar ist, aber das Risiko im Vergleich zu Patienten mit einem milderem Vitium erhöht ist (Pasquali et al. 2007b).

Elkins et al. berichteten 2008 von ihren Erfahrungen mit 487 Patienten im Alter zwischen 2 Tagen und 62 Jahren (Mittelwert 24 Jahre), davon 197 unter 18 Jahren. Die Krankenhausletalität betrug 3,9\% (19/487 Patienten). Unter den operierten Patienten befanden sich 40 mit einer präoperativen floriden Endokarditis. Im Langzeitverlauf traten 15 späte Todesfälle auf, von denen 8 Herzklappen-assoziiert waren, es wurde nicht zwischen Autograft und Homograft unterschieden. 7 Todesfälle hatten keine kardiologische Ursache. Somit ergab sich eine durchschnittliche 16-Jahres-Überlebensrate von 84\% $( \pm 8 \%)$ und eine Freiheit von Klappen-assoziierten Todesfällen von 89\% ( $\pm 4 \%)$ nach 16 Jahren (Elkins et al. 2008).

Bei den von Kadner et al. 2008 untersuchten Patienten kam es bei den 52 operierten Kindern und Jugendlichen zu 8 Todesfällen, wobei 2 dieser Patienten eine Ross-KonnoOperation erhalten hatten. 5 Todesfälle traten im postoperativen stationären Aufenthalt auf. Die Ursache war bei 4 Patienten ein akutes linksventrikuläres Pumpversagen, in einem Fall ein septischer Schock. 3 Patienten verstarben im poststationären Verlauf aus nicht näher bezeichneten Gründen. Alle betroffenen Kinder wiesen ein Alter unter 2 Mo- 
naten auf. Im weiteren Nachbeobachtungszeitraum waren 3 Patienten an nicht-kardialen Todesursachen verstorben, sodass ein 10-Jahres-Überleben von $90 \%( \pm 4 \%)$ resultierte. Hieraus leiteten die Autoren ab, dass ein sehr junges Operationsalter ein Risikofaktor für ein erhöhtes Letalitätsrisiko ist (Kadner et al. 2008).

In der 2009 erschienenen Veröffentlichung von Brown et al. über die Behandlung von 97 Kindern und Erwachsenen, bei denen zwischen 2001 und 2009 eine Ross-Operation durchgeführt worden war, wurden bei 3 Patienten folgende Komplikationen beschrieben: Bei 2 Patienten waren Revisionsoperationen notwendig, eine aufgrund einer konservativ nicht zu stillenden Blutung im Operationsgebiet, die zweite aufgrund einer Kompromittierung der rechten Koronararterie. 1 weiterer Patient entwickelte postoperativ ein Kompartmentsyndrom des linken Beines (Brown et al. 2010).

Valeske et al. berichteten von 2 frühen Todesfällen bei ihren zwischen 1996 und 2008 operierten 98 Patienten. Bei beiden verstorbenen Patienten lagen komplexe Fehlbildungen des LVOT vor. 1 Kind war notfallmäßig im beginnenden Linksherzversagen operiert worden. Die Gesamtüberlebensrate aller Operierten lag bei 98\% (Valeske et al. 2010). Bekkers et al. veröffentlichten 2010 Ergebnisse von 155 Patienten, die zwischen 1988 und 2009 eine Ross-Operation erhalten hatten, darunter 47 Patienten im Alter unter 16 Jahren (mittleres Alter 21,4 $\pm 13,7$ Jahre). Die 30-Tages-Mortalität betrug 2,6\% (4 Patienten). 2 Patientinnen verstarben an kardialen Ursachen, eine 40-jährige an akutem linksventrikulärem Pumpversagen und ein 4 Monate alter Säugling auf Grund von schweren Arrhythmien. Ein 26-jähriger Mann erlitt eine Lungenembolie und eine 24-jährige Patientin mit Turner Syndrom eine tödliche Sepsis in Folge einer Mediastinitis. Im weiteren Langzeitverlauf verstarben noch 7 Patienten, davon 3 an Herzklappen-bedingten Komplikationen und 4 aus anderer Ursache. Ein 12-jähriges Mädchen erlitt nach präoperativ aufgetretenem rheumatischem Fieber eine tödliche Mitralinsuffizienz. Die beiden anderen Todesfälle wurden nicht genauer beschrieben. Das 15-Jahres-Überleben betrug somit $92 \%( \pm 1,8 \%)$ (Bekkers et al. 2010).

Die 2010 publizierten Ergebnisse des europäischen Ross-Registers von 152 Patienten im Alter unter 16 Jahren wiesen eine Krankenhaus-Mortalität von 2,6\% auf. Als Risikofaktoren wurden ein gleichzeitiger Mitralklappenersatz und eine zuvor bestehende Aortenstenose identifiziert. Im Langzeitverlauf verstarben 7 Patienten, 6 davon aufgrund kardialer Ereignisse. Als Risikofaktoren für einen späten postoperativen Tod konnten eine zuvor abgelaufene Endokarditis, eine gleichzeitig vorgenommene koronare Bypass-Operation und eine lange Perfusionszeit mit EKZ ermittelt werden. Das 10-Jahres-Überleben lag bei $90.4 \%$ ( $\pm 3,1 \%)$ (Hörer et al. 2010). 
Die in Göttingen aufgetretenen postoperativen Komplikationen lassen sich einteilen in:

- unmittelbar durch die Operation bedingte Komplikationen (Pleuraerguss, chirurgisch bedingte Blutung, neurologische Komplikationen),

- kardiale Komplikationen (Rhythmusstörungen, Perikarditis) und

- andere durch die maschinelle Beatmung oder Immobilisation aufgetretenen Komplikationen.

Nur wenige frühere Studien haben die nicht-klappenbedingten Komplikationen detailliert beschrieben. Rhythmusstörungen wie insbesondere ein AV-Block II. oder III. Grades können nach der Operation persistieren, so dass eventuell die Implantation eines Schrittmachers notwendig wird.

Bei einigen Patienten wurden Fieber und Entzündungszeichen beobachtet, die als Hinweis auf eine immunologische Reaktion auf das körperfremde Material des Homografts gedeutet werden konnte. Durch frühzeitige antiinflammatorische und teils auch immunsuppressive Therapie mittels Kortison konnte die Symptomatik beseitigt werden.

Von anderen Autoren beschriebene Komplikationen an den Anastomosen der Koronarostien am Autograft traten bei uns nicht auf. Auch ein Frühversagen des Autografts oder des Homografts/Xenografts konnten wir nicht beobachten. Alle beschriebenen Komplikationen konnten beherrscht und erfolgreich therapiert werden. Daraus kann abgeleitet werden, dass die Ross-Operation in der in Göttingen durchgeführten und etablierten Form ein sicheres Verfahren ist.

Die Operationsletalität der in Göttingen operierten Patienten (1/30 entsprechend 3,3\%) entsprach der in der Literatur beschrieben niedrigen Letalitätsrate. Die einzige verstorbene Patientin wies keine der in anderen Studien ermittelten Risikofaktoren wie ein sehr junges Operationsalter, eine zuvor abgelaufene oder eine floride Endokarditis auf. Es lag ein kombiniertes Vitium der Aortenklappe mit resultierender extremer Hypertrophie des linken Ventrikels vor. Bei dem Eingriff handelte es sich um eine einfache Ross-Operation, die nicht mit anderen Eingriffen kombiniert war. Ursächlich für die postoperative ventrikuläre Rhythmusstörung kommt am ehesten eine ungenügende Myokardprotektion in Frage. Warum es unter der postoperativen antiarrhythmischen und herzunterstützenden Therapie mit einem ECMO-System zu einer Hirnblutung kam, die zum Hirntod führte, blieb ungeklärt. Letztendlich ist nicht zu klären warum die Patientin verstorben ist. 
Die guten Ergebnisse lassen sich mit der strengen Indikationsstellung erklären. Stellte sich intraoperativ ein zu großes Missverhältnis der Durchmesser zwischen PK und AK dar oder wurde eine zuvor nicht bekannte Fehlbildung der PK entdeckt, so entschied man sich zu einem alternativen Verfahren des AKE.

Auch sind in dem Göttinger Patientenkollektiv keine Patienten vertreten, für die in anderen Studien ein erhöhtes Letalitätsrisiko festgestellt worden war: keiner der Patienten wies eine floride Endokarditis auf oder war in der Vorgeschichte daran erkrankt. Alle Eingriffe waren elektiv. Es wurden zwar teilweise andere korrigierende Operationen wie Septomyektomie nach Morrow, subvalvuläre Inzision nach Bigelow, ASD-Verschluss, Ductus arteriosus-Verschluss und Schrittmacherwechsel (vergleiche Tabelle 3) gleichzeitig mit der Ross-Operation durchgeführt, darunter jedoch war kein Ersatz einer anderen erkrankten Herzklappe, was vom europäischen Ross-Register als Risikofaktor benannt wird. 2 Patienten wiesen zum Zeitpunkt der Operation ein Alter unter 2 Jahren auf (sechs Monate, bzw. 14 Monate). Ansonsten lag bei den Göttinger Patienten keiner der in anderen Studien beschriebenen Risikofaktoren vor.

\subsection{Die postoperative Entwicklung des pulmonalen Autografts}

Ein Kernpunkt in der Diskussion um die Effizienz der Ross-Operation ist bis heute die Frage nach der Funktionstüchtigkeit und der Haltbarkeit des pulmonalen Autografts im Langzeitverlauf. Es wird grundsätzlich angenommen, dass die körpereigene PK das beste verfügbare Aortenklappensubstitut für Kinder und Jugendliche darstellt. Im Gegensatz zu allen anderen verfügbaren Implantaten hat der Autograft das Potential zum somatischen Wachstum und damit zur bedarfsgerechten Anpassung an die linksventrikuläre Ausflussbahn. Nach mehreren Jahrzehnten Erfahrung mit dieser Operationstechnik stellt sich heute die Frage, ob es sich bei der nach langjähriger Beobachtung im Echokardiogramm registrierten Erweiterung der Neo-Aortenwurzel nach Ross-Operation um ein synchron zur Körperentwicklung altersentsprechendes physiologisches Wachstum handelt oder diese eine Dilatation mit im Verlauf auftretender behandlungsbedürftiger Insuffizienz darstellt.

Verschiedene Arbeitsgruppen haben nachgewiesen, dass das Wachstum der Herzstrukturen des Menschen im proportionalen Verhältnis zur Zunahme der Körperoberfläche steht (Henry et al. 1978, Snider et al. 1984). Zur Bewertung unserer Ergebnisse im Bezug auf die Differenzierung zwischen Dilatation bzw. Wachstum des Autografts muss Bezug auf Normkurven gesunder Kollektive in Form von Z-Scores genommen werden, wie dies auch 
in anderen aktuellen Veröffentlichungen zu dieser Frage geschieht (Hörer et al. 2009). Auch die Arbeitsgruppe von Solymar versuchte, diese Frage zu beantworten. Sie verglichen die echokardiografische Größenentwicklung der Aortenklappen von 8 Kindern, bei denen auf Grund einer kritischen Aortenstenose im frühen Lebensalter (mittleres Alter bei Operation 6,4 Monate) eine Ross-Operation durchgeführt worden war, mit den Messwerten von 12 gesunden Kindern. Der mittlere Nachbeobachtungszeitraum der gesunden Kinder betrug 4,9 Jahre, der der Kinder nach Ross-Operation 5,2 Jahre. Die Untersuchung zeigte, dass es im ersten postoperativen Jahr zunächst zu einer im Verhältnis zum Körperwachstum übermäßigen Größenzunahme des Autografts kam und damit eine Dilatation entstand. Im weiteren Verlauf glich sich jedoch die Größenzunahme dem Körperwachstum an, so dass eine physiologische somatische Größenzunahme nach dem ersten Jahr vermutet wurde (Solymar et al. 2000).

Ohye et al. beschäftigten sich ebenfalls mit dem Wachstum des Autografts im Langzeitverlauf. Hierzu maßen sie im Zeitraum von 1993 bis 2000 die echokardiographische Entwicklung des Autografts bei 10 Patienten, bei denen im Alter von unter einem Jahr eine Ross-Konno-Operation durchgeführt worden war. Sie bestimmten die Z-Werte für den Aortenklappenring und den sino-tubulären Übergang in den ersten 12 Monaten und ein weiteres Mal nach einem Zeitraum von mehr als 36 Monaten nach der Operation (Mittelwert 66 Monate). Es zeigte sich, dass es im Bereich des Aortenklappenringes kaum zu Veränderungen der Z-Werte kam. In den ersten 12 Monaten betrug der Mittelwerte für die Z-Wert-Veränderung -0,6 $\pm 0,6$, für die Messung über 36 Monate nach Operation betrug diese -0,4 $\pm 0,7$. Bei den Messungen des sino-tubulären Überganges zeigte sich dagegen eine signifikante Zunahme des Durchschnitts der Z-Werte im Vergleich zum Normalkollektiv innerhalb der ersten 12 Monate von $+2,1 \pm 1,6$. Diese Zunahme stabilisierte sich jedoch im weiteren Beobachtungszeitraum (Z-Wert-Veränderung von 12 bis 36 Monaten $0,6 \pm 0,9)$. Obwohl sich bei einigen Patienten Autograftinsuffizienzen im weiteren Verlauf entwickelten, musste bei keinem ein Austausch der Neo-Aortenklappe erfolgen (Ohye et al. 2001).

Im Jahre 2004 veröffentlichten Kouchoukos et al. die Ergebnisse von 119 Jugendlichen und jungen Erwachsenen (mittleres Alter bei Operation: 31 Jahre), bei denen zwischen 1989 und 2001 eine Ross-Operation durchgeführt worden war. Es wurden die Durchmesser des Aortenklappenrings, des sino-tubulären Übergangs und der Aortenwurzel gemessen. Außerdem wurde der Grad der gegebenenfalls bestehenden Insuffizienz der Neo-Aortenklappe echokardiografisch bestimmt. Es zeigte sich eine signifikante Zunahme der Durchmesser des sino-tubulären Überganges und der Aortenwurzel. Dagegen 
wies der Aortenklappenring keine signifikante Erweiterung auf. Bei 8 Patienten bestand eine zweitgradige, bei 15 eine erstgradige Insuffizienz. Insgesamt zeigte sich eine Proportionalität zwischen der Dauer des Beobachtungszeitraumes und sowohl dem Auftreten einer Klappeninsuffizienz als auch der signifikanten Erweiterung der Durchmesser der Autograftwurzel (Kouchoukos et al. 2004).

Williams et al. publizierten 2005 Ergebnisse von Ross-Operationen im Säuglingsalter. In dieser Studie wurde von 27 Patienten berichtet, die im Zeitraum zwischen 1991 und 2004 wegen einer Aortenstenose operiert worden waren. Alle Säuglinge waren jünger als 18 Monate. 10 der Patienten hatten zuvor eine Ballonvalvuloplastie erhalten. Im Nachbeobachtungszeitraum von durchschnittlich 6,1 Jahren wurde bei keinem der Patienten eine erneute Operation der Aortenklappe notwendig. Echokardiographisch wurde im Kontrollzeitraum bei 1 Patienten eine Autograftstenose und bei 4 Patienten eine Autograftinsuffizienz nachgewiesen. Die Auswertung der Z-Werte an den Messpunkten Autograftring und sino-tubulärer Übergang zeigten, dass es zwar im ersten postoperativen Jahr zu einer signifikanten Zunahme der Durchmesser kam, dass diese Veränderung im weiteren Verlauf aber nicht mehr zu beobachten war (Williams et al. 2005).

Die Arbeitsgruppe von Pasquali fasste die Ergebnisse von 74 Patienten im Alter zwischen 3 Tagen und 34 Jahren (Mittelwert 9 Jahre), bei denen im postoperativen Verlauf über einen Nachbeobachtungszeitraum von durchschnittlich 4,7 Jahren (von 3 Monaten bis 9,3 Jahren) 2 oder mehr echokardiographische Nachuntersuchungen durchgeführt worden waren. Mit Hilfe dieser Echokardiographien wurde anhand von Z-Werten eine Dilatation am Aortenklappenring, am sino-tubulären Übergang und an der Aortenwurzel nachgewiesen. Bei 9 Patienten musste ein erneuter Eingriff an der Aortenklappe aufgrund dieser Dilatation durchgeführt werden. Als die Re-Operation begünstigende Faktoren konnten langer Beobachtungszeitraum, ein vor der Operation bestehender und chirurgisch korrigierter Ventrikel-Septum-Defekt und eine vor der Ross-Operation durchgeführte andere Form des Aortenklappenersatzes identifiziert werden. Es zeigte sich jedoch, dass das Auftreten einer Dilatation nicht mit dem Auftreten einer Autograftinsuffizienz und der Notwendigkeit eines erneuten Aortenklappenersatzes korrelierte (Pasquali et al. 2007a). Stewart et al. befassten sich 2007 mit der Frage, ob in ihrem Patientenkollektiv von Ross-Operierten mit einem Durchschnittsalter von 12,9 Jahren (von 14 Monaten bis 21 Jahren) durch eine zusätzliche Anuloplastie des Autografts, das Auftreten einer Regurgitation verhindert werden konnte. Die echokardiographischen Nachuntersuchungen zeigten eine signifikante Zunahme des Autograftring- und des Autograftwurzel-Durchmessers sowohl bei Patienten mit als auch ohne Anuloplastie. Auch die Anzahl erneut notwendi- 
ger Eingriffe an der Aortenklappe war in beiden Patientengruppen gleich groß (Stewart et al. 2007).

Kadner et al. publizierten Ergebnisse von 52 Patienten, die vor dem 16. Lebensjahr mittels Ross- oder Ross-Konno-Operation behandelt worden waren. Der mittlere Nachuntersuchungszeitraum betrug 43 Monate. Von diesen Patienten musste in einem Fall wegen einer bedeutsamen Insuffizienz der Austausch des Autografts bereits nach 6 Monaten erfolgen. Eine milde Autograftinsuffzizienz entwickelte sich im Verlauf bei 24 Patienten, eine mittelgradige bei 1 Patienten. Es zeigte sich, dass in dem Einzelfall des Autograftversagens die Morphologie der verwendeten PK pathologisch war. In der postoperativen Entwicklung gab es keinen signifikanten Unterschied der Neo-Aortenklappen zwischen den Patienten nach klassischer Ross und Ross-Konno-Operation (Kadner et al. 2008).

Elkins et al. identifizierten in ihrer schon erwähnten Studie, die 487 Patienten einschloss, darunter 197 Patienten unter 18 Jahren, bei $20 \%$ der Patienten 2 Risikofaktoren für ein Versagen der Neo-Aortenklappe: einerseits das männliche Geschlecht, andererseits eine vor der Operation bestehende AI. Die Bestimmung der Durchmesser des sino-tubulären Übergangs und der Autograftwurzel sowie die Berechnung der jeweiligen Z-Werte ergab wie schon wie bei anderen Studien eine signifikante Zunahme der Messwerte innerhalb der ersten 12 Monate nach Operation. Risikofaktoren für eine frühe Re-Operation waren eine früh postoperativ auftretende AI zweiten Grades und ein Ventrikel-Septum-Defekt in der Vorgeschichte. Nach 12 Monaten war auch bei diesen Autoren keine relevante Dilatation mehr festzustellen (Elkins et al. 2008).

Hörer et al. veröffentlichten 2009 die Ergebnisse von 152 Patienten jünger als 16 Jahre, bei denen eine Ross-Operation durchgeführt worden war. In einem Nachbeobachtungzeitraum von 6,1 \pm 4,2 Jahren, benötigten 7 Patienten (4\%) einen erneuten Eingriff am Autograft (davon sechsmal einen Austausch und einmal eine Rekonstruktion). Als Risikofaktoren wurden eine zuvor abgelaufene Endokarditis, eine primäre AI und eine lange Nachbeobachtungszeit ermittelt (Hörer et al. 2009).

Valeske et al. veröffentlichten 2010 ihre Operationsergebnisse mit Schwerpunkt auf der Entwicklung des pulmonalen Autografts. Hierzu wurden die Daten von 98 Kindern, die zwischen 1996 und 2008 operiert worden waren, ausgewertet. Das mittlere Alter bei Operation betrug 11 Jahre. Es wurden Messdaten aus Echokardiographien verwendet, die nach Studienprotokoll im ersten Jahr alle 3-6 Monate und danach alle 6-12 Monate wiederholt wurden. Dabei konnten Grad der vorhandenen Autograftinsuffizienz bestimmt und außerdem Z-Werte für die Durchmesser des sino-tubulären Überganges berechnet werden. Im spät-postoperativen Verlauf zeigten 70 Patienten eine regelrechte 
Autograft-Funktion, 22 eine Insuffizienz ersten Grades, 3 eine Insuffizienz zweiten Grades und 1 Patient eine Insuffizienz dritten Grades, die das Einsetzen einer mechanischen Kunstklappe erforderlich machte. Anhand der berechneten Z-Werte war eine übermäßige Dilatation des Autografts nur bei 10 Patienten nachzuweisen. 86 wiesen diese morphologische Veränderung nicht auf. In 80\% der Fälle kam es im Verlauf von bis zu 12 Jahren nach Operation nicht zu einer Dilatation (Valeske et al. 2010).

Bei den Göttinger Patienten kam es im postoperativen Verlauf nach Auswertung der Messwerte des Autografts in keinem Fall zu einer Dilatation. Trotzdem war im Langzeitverlauf eine Zunahme der Entwicklung von meist erstgradigen Autograftinsuffizienzen festzustellen. Nur 1 Patient benötigte eine erneute Operation am Autograft. Wie bei den Patienten von Pasquali et al. gab es auch bei den Göttinger Patienten keine Korrelation zwischen Dilatation des Autografts und dem Auftreten von einer Autograftinsuffizienz. Bei den Betroffenen war im beobachteten Zeitraum keine Intervention notwendig, da die Klappeninsuffizienzen jeweils nur leichtgradig und ohne hämodynamische Relevanz waren.

Als Risikofaktoren wurden in den oben zitierten Studien die wachsende Nachbeobachtungsdauer, eine vor Operation bestehende AI, eine vorangegangene Endokarditis, ein Ventrikelseptumdefekt und eine pathologisch veränderte PK bestimmt. Das in der vorliegenden Studie beobachtete Patientenkollektiv ist zu klein, um diese Risikofaktoren zu überprüfen oder weitere Faktoren statistisch valide zu erfassen. Wurde intraoperativ festgestellt, dass die PK aufgrund einer übermäßigen Differenz des Durchmessers zur AK oder einer Fehlbildung entgegen der präoperativen Diagnostik nicht geeignet war, so wurde alternativ ein anderes Klappensubstitut eingesetzt und keine Ross-Operation durchgeführt. Auch hatte keiner der in Göttingen operierten Patienten eine abgelaufene Endokarditis in der Vorgeschichte. Somit kamen 2 der identifizierten Risikofaktoren nicht vor.

Einschränkend bei der Bewertung der Z-Scores ist zu nennen, dass wegen der unterschiedlichen Zeitpunkte der Durchführung der postoperativen Echokardiographien im Vergleich der Patienten zeitliche Überschneidungen zwischen Messung 1 und 2 entstehen. Bei manchen Patienten, aus den frühen Operationsjahren, liegt der Zeitpunkt der ersten verfügbaren Echokardiographie deutlich hinter dem zweiten Messzeitpunkt bei den später operierten Patienten. Damit wird die Aussagekraft der Messergebnisse eingeschränkt. Die Feststellung, dass es bei keinem der Patienten im Spätverlauf zu einer Dilatation kam, hat trotz dieser Einschränkung Bestand. Um eine bessere Aussage über 
die Haltbarkeit der Autografts treffen zu können, müssen weitere Jahre der Nachbeobachtung abgewartet werden.

Betreffend der Haltbarkeit des Autografts in diesem beobachteten Patientenkollektiv kann festgestellt werden, dass es in einer maximalen Nachbeobachtungszeit von 15 Jahren nicht zu einer Beeinträchtigung der Herzfunktion auf Grund eines Autograftversagens kam. Ob fehlende Risikofaktoren bei der Auswahl des Patientenkollektivs und sorgfältige Patientenauswahl bzw. Indikationsstellung dafür mitverantwortlich sind ist in diesem Rahmen nicht zu beantworten.

\subsection{Die postoperative Entwicklung des linken Ventrikels}

Neben der Frage, wie sich die operativ ausgetauschte Klappe im weiteren Lebensverlauf verhält, ist die Entwicklung des linken Ventrikels bisher wenig untersucht worden. Eine Obstruktion des LVOT, insbesondere valvuläre und subvalvuläre, sowie die AI sind neben der arteriellen Hypertonie die wesentlichen Risikofaktoren für eine ventrikuläre Hypertrophie. So hat die Funktion eines Klappenimplantates einen unmittelbaren Einfluss auf die weitere Entwicklung des linken Ventrikels. Besonders bei Kindern und Jugendlichen mit ihrer langen Lebenserwartung hat die Entwicklung des linken Herzens für die Langzeitprognose eine besonders große Bedeutung.

Über mechanische Klappenprothesen weiß man, dass sie bei engem Klappenring zu einem Prothesen-Patienten-Mismatch mit Reststenose und daraus resultierender erhöhter Nachlast führen können. Daraus können sich sekundäre Myokardschäden wie ventrikuläre Hypertrophie (Pibarot und Dumesnil 2000, Duebener et al. 2005) und im Langzeitverlauf Folgeerkrankungen wie relative Koronarinsuffizienz und Herzinsuffizienz ergeben (Koren et al. 1991). Biologische Aortenklappenprothesen haben bessere hämodynamische Eigenschaften. Der pulmonale Autograft nimmt innerhalb der Gruppe der Klappenbioprothesen eine Sonderstellung ein (Pibarot und Dumesnil 2000). Es besteht die Hoffnung, dass sich die Ross-Operation positiv auf die Langzeitprognose der Patienten im Hinblick auf kardiale Ereignisse durch sekundäre Schäden des linken Ventrikels auswirkt.

In der Arbeit von Brown et al. aus dem Jahre 2001 über die Untersuchung von 50 operierten Kindern und Jugendlichen wurde echokardiographisch die Entwicklung der linksventrikulären Durchmesser und der linksventrikulären Masse nach Ross-Operation gemessen. Es zeigte sich eine Abnahme dieser Durchmesser, so dass als Resumee die 
Ross-Operation als ein gutes Verfahren zum AKE eingestuft wurde (Brown et al. 2001). Schmid et al. veröffentlichten 2002 Daten postoperative Ergebnisse von 42 erwachsenen Patienten (mittleres Alter 36 \pm 6 Jahre). Mittels Echokardiographie und Myokardszintigrafie wurden die linksventrikulären Durchmesser bestimmt und die Funktion des linken Ventrikels bewertet. Es kam postoperativ zu einer statistisch signifikanten Abnahme der linksventrikulären Masse innerhalb der ersten sechs Monate nach Operation. Ebenso kam es in diesem Zeitraum zu einer signifikanten Abnahme der Durchmesser sowohl des IVS als auch der HW des linken Ventrikels. Auch der LVEDD nahm ab, jedoch ohne statistisch nachweisbare Signifikanz (Schmid et al. 2002).

Im Jahre 2005 veröffentlichten Duebener et al. Daten aus dem Deutschen Ross-Register von Patienten mit einem Durchschnittsalter von 43,6 Jahren (insgesamt 646 jugendliche und erwachsene Patienten im Alter von 16 bis 71 Jahre). Untersucht wurde die Reduktion der linksventrikulären Masse nach Ross-Operation anhand von postoperativen Echokardiographien. Die Patienten wurden nach Operationstechnik in zwei Gruppen (subcoronare Technik vs. Wurzelersatz) eingeteilt. Es zeigte sich eine signifikante Abnahme der linksventrikulären Masse in beiden Gruppen im ersten Jahr nach Ross-Operation. Im weiteren Verlauf stagnierte die Entwicklung in beiden Gruppen. Als Risikofaktoren für eine inkomplette Rückentwicklung wurden Nikotinabusus und diastolische Hypertension festgestellt (Duebener et al. 2005).

Pasquali et al. veröffentlichten 2007 ebenfalls Daten über die Entwicklung der echokardiographisch bestimmten Durchmesser des linken Ventrikels. Die Patienten dieser Studie mit einem Durchschnittsalter von 9 Jahren zum Zeitpunkt der Operation zeigten eine Reduktion des LVEDD. Es zeigte sich eine signifikante Reduktion des Z-Wertes. Vor Operation zeigten $43 \%$ der Patienten einen Z-Wert von über 2, in der Nachuntersuchung waren es nur noch 18\% der Patienten (Pasquali et al. 2007a).

Bei den Göttinger Patienten zeigten sich bei der zweiten postoperativen Kontrolle gleichbleibende Z-Werte für den LVESD, das IVS und die linksventrikuläre HW, eine signifikante Abnahme des Z-Wertes für den LVEDD. Als Risikofaktoren für die inkomplette Rückentwicklung der linksventrikulären Masse vermuteten Duebener et al. Nikotinabusus und diastolische Hypertension (Duebener et al. 2005). Beide Faktoren wurden im Göttinger Patientenkollektiv nicht abgefragt und statistisch erfasst.

Die Ergebnisse zeigten, dass es beim untersuchten Patientenkollektiv nach Ross-Operation erwartungsgemäß nicht zu einer fortschreitenden Dilatation oder Hypertrophie des linken Ventrikels kam. Die Zunahmen der Durchmesser entsprachen dem somatischen Wachs- 
tum und glichen sich an die Vergleichswerte Gesunder an. Eine gesonderte Aussage zur Ross-Konno-Operation ist bei nur zwei betroffenen Patienten statistisch nicht zu gewinnen, es lässt sich jedoch von insgesamt ebenfalls sehr guten postoperativen Ergebnissen sprechen.

\subsection{Die Entwicklung des Homografts/Xenografts als Pulmonalklappensubstitut}

Ein grundsätzlicher Nachteil der Ross-Operation im Vergleich zu anderen Operationsverfahren ist die Behandlung einer univalvulären Erkrankung mittels eines bivalvulären Eingriffs. Dies zieht erneute Eingriffe oder Operationen des RVOT auf Grund der begrenzten Haltbarkeit von Homografts/Xenografts auch im Niederdrucksystem des kleinen Kreislaufs nach sich. Im Allgemeinen ist die Haltbarkeit der bei einer Ross-Operation eingesetzten Pulmonalis- Homografts besser als bei Implantation aus anderen Indikationen, da das Substitut in einen initial gesunden RVOT mit normalen pulmonalen Widerstandsverhältnissen eingesetzt wird. Als ein Grund für die Verkalkung und Stenosierung des Homograft/Xenografts wird eine immunologische Reaktion auf das körperfremde Gewebe vermutet (Brockbank und Song 2003).

Im Jahre 1999 veröffentlichten Niwaya et al. die Ergebnisse von 331 Patienten mit einem mittleren Alter von 14 Jahren (zwischen 2 Tagen und 62 Jahren), denen zwischen 1985 und 1997 ein kryokonservierter Pulmonalis- oder Aorten- Homograft als Pumonalklappenersatz (PKE) eingepflanzt worden war. Der mittlere Nachbeobachtungszeitraum betrug 3,8 Jahre (1 Monat bis 11,2 Jahre). Den größten Teil der Operierten machten mittels Ross-Operation versorgte Kinder aus, es wurden jedoch auch Patienten mit PKE bei Fallot-Tetralogie (41 Fälle), Truncus arteriosus communis (11 Fälle), Transposition der großen Gefäße (11 Fälle) und bei anderen Krankheitsbildern berücksichtigt. Es zeigte sich, dass die Ross-Patienten ein signifikant niedrigeres Risiko für eine frühe Degeneration des Conduits aufwiesen. Dies wurde dadurch erklärt, dass bei ursprünglich gesunder PK weder eine Dilatation bzw. Deformierung des RVOT noch ein pulmonaler Hypertonus vorlägen und somit das Risiko für die Entwicklung einer Klappendegeneration natürlicherweise niedriger sei als bei schon vorbestehenden Pathologien der PK und den daraus resultierenden strukturellen Veränderungen. Bei 39 Patienten war im Nachuntersuchungszeitraum eine Dysfunktion des Conduits aufgetreten, bei 29 ( 9\%) musste der 
Homograft nach einem Intervall von bis zu 8 Jahren postoperativ ausgetauscht werden, bei 39 kam es zu einer Dysfunktion mit einem transvalvulärem Druckgradienten von über $40 \mathrm{mmHg}$. Die Studie benannte als Risikofaktoren für ein frühes Homograft-Versagen die Verwendung von kryokonservierten Aorten-Homografts, ein junges Spenderalter und einen späten Operationszeitpunkt. Der kryokonservierte Pulmonalis-Homograft erwies sich als haltbarster PKE (Niwaya et al. 1999).

Javadpour et al. publizierten 2002 eine Untersuchung, die sich mit der Frage beschäftigte, ob die Kalzifikation und Degeneration eines Homografts in Pulmonalis-Postition auf besondere Eigenschaften des Empfänger- oder des Spendergewebes zurückzuführen sei. Hierzu waren die Langzeitergebnisse von 60 Kindern herangezogen worden, die mit unterschiedlichsten Indikationen einen Homograft als PKE implantiert bekommen hatten. Es waren 24 Pulmonalis-Homografts und 26 Aorten-Homografts verwendet worden, wobei 24 dieser Conduits von bereits verstorbenen Spendern entnommen worden waren. Die anderen 26 Klappen stammten von hirntoten Spendern und waren aus dem noch schlagenden Herzen ("heart beating donors") entnommen worden. Die 50 Patienten, die diese Operation überlebten, zeigten bei Nachuntersuchungszeitraum von 3 bis 108 Monaten in 10 Fällen echokardiographisch nachweisbare Verkalkungen des Homografts, wobei es sich in allen Fällen um Aorten-Homografts handelte. Somit war der Unterschied zwischen Aorten-Homografts und Pulmonalis-Homografts signifikant $(\mathrm{P}=0,0006)$. Die Entnahme bei lebenden oder verstorbenen Spendern zeigte für das Degenerationsrisiko keinen Unterschied (Javadpour et al. 2002).

Die 2001 von Ohye et al. veröffentlichten Ergebnisse von 10 Patienten im Alter von unter 12 Monaten, die eine Ross-Konno-Operation erhalten hatten, zeigten, dass auch beim sehr jungen, rasch wachsenden Patienten die Art des verwendeten Homografts von Bedeutung für eine lang anhaltende gute Funktion ist. Die 2 während des Nachbeobachtungszeitraums von 1993 bis 2000 notwendig gewordenen Re-Operationen betrafen 1 Patienten mit Aorten-Homograft und 1 Patienten, bei dem der verwendete PulmonalisHomograft vor der Implantation verkleinert worden war. Bei den restlichen Patienten, von denen 7 einen nicht verkleinerten Pulmonalis-Homograft und 1 einen nicht näher beschriebenen Xenograft erhalten hatte, war es nicht zu behandlungsbedürftigen Stenosen oder Insuffizienzen gekommen (Ohye et al. 2001).

Williams et al. berichteten 2005 von 27 Patienten, bei denen ebenfalls zu einem sehr frühen Zeitpunkt (im Alter unter 18 Monaten) eine Ross-Operation durchgeführt worden war. Es wurden zum PKE insgesamt 15 Pulmonalis-Homografts, 10 Aorten-Homografts und 2 porcine Xenografts verwendet. Die Durchmesser der Conduits lagen zwischen $9 \mathrm{~mm}$ 
und $20 \mathrm{~mm}$. In einem medianen Nachbeobachtungszeitraum von 6,1 Jahren musste bei insgesamt 4 Patienten der Homograft bzw. Xenograft ausgetauscht werden. Bei 2 Patienten handelte es sich um klappentragende Conduits porciner Herkunft, die bereits nach 13 Monaten bzw. 4,5 Jahren ausgetauscht werden mussten. Daraufhin wurden keine weiteren porcinen Klappen mehr verwendet. Die 2 anderen austauschbedürftigen PulmonalisConduits waren Homografts, die nach 9 bzw. 10 Jahren ausgewechselt werden mussten. $87 \%$ der Patienten blieben binnen 8 Jahren von Re-Operationen verschont(Williams et al. 2005).

Elkins et al. veröffentlichte 2008 Ergebnisse von Nachuntersuchungen bei 487 Patienten mit einem mitteleren Alter von 24 Jahren (Altersspanne: 2 Tage bis 62 Jahren) über einen Zeitraum von 16 Jahren. Es wurden, wenn verfügbar, Pulmonalis-Homografts verwendet. Der Durchmesser des Allografts wurde anhand des Patientenalters festgelegt und war im Schnitt größer, als der anhand der BSA errechnete Durchmesser. Bei $33 \mathrm{~Pa}-$ tienten mussten innerhalb des Nachuntersuchungszeitraums der Allograft ausgetauscht werden oder aber Interventionen wie z.B. eine Stent-Implantation durchgeführt werden. Aus diesem Kollektiv blieben $82 \%( \pm 4 \%)$ der Patienten im Verlauf von 16 Jahren frei von Re-Operationen (Elkins et al. 2008).

Pasquali et al. stellten 2007 die Ergebnisse von 121 Kindern mit einem mittleren Alter von 8,2 Jahren (Alterspanne: 3 Tage bis 34 Jahre) zum Zeitpunkt der Ross-Operation vor, die zwischen 1995 und 2004 operiert worden waren. Als PKE waren 113 PulmonalisHomografts und 8 Aorten-Homografts eingesetzt worden. In dieser Studiengruppe blieben $82 \%$ von Re-Interventionen an der Neo-PK verschont. Als Risikofaktoren für eine erneut notwendige Operation wurden eine kleine Größe des Homografts, die Verwendung von Aorten-Homografts, ein jüngeres Patientenalter bei der Ross-Operation und eine lange intraoperative kardio-pulmonale Bypasszeit benannt (Pasquali et al. 2007b). Hörer et al. veröffentlichten 2010 die Langzeitergebnisse von 152 operierten Kindern über einen Nachuntersuchungszeitraum von bis zu 16 Jahren. Im Nachuntersuchungszeitraum benötigten 17 Patienten eine Nachoperation des Conduits in pulmonaler Position. In diesem Kollektiv bestand die Notwendigkeit einer Re-Operation in 79,6\% $( \pm 6,1 \%)$ innerhalb von 10 Jahren. Als Risikofaktoren für die operationsbedürftige Homograftstenosierung wurden die Verwendung eines Aorten-Homografts (3-fache Risikoerhöhung im Vergleich zum Pulmonalis-Homograft) und ein kleiner Conduitdurchmesser herausgearbeitet (Hörer et al. 2010).

Valeske et al. beschrieben die Ergebnisse von 98 Patienten über einen Nachuntersuchungszeitraum von bis zu 12 Jahren (Altersspanne: 54 Tage bis 15 Jahre). Von den 
10 verwendeten kryokonservierten Aorten-Homografts mussten 5 ausgetauscht werden mussten. Auch der einzige verwendete Conduit mit porciner Klappe musste aufgrund von rascher Stenosierung ausgetauscht werden. Die 72 eingepflanzten Pulmonalis-Homografts und die 15 verwendeten Contegra ${ }^{\circledR}$-Xenografts (bovine Jugularvene) zeigten dagegen über den Beobachtungszeitraum eine gute Funktion. Darum empfahlen die Autoren die Verwendung von kryokonservierten Pulmonalis-Homografts und bei jüngeren Patienten mit kleineren Durchmessern des LVOT die Verwendung von bovinen JugularvenenXenografts (Contegra ${ }^{\circledR}$ ) (Valeske et al. 2010).

Bei den in Göttingen operierten 30 Patienten zeigte sich im Beobachtungszeitraum eine Progredienz der den Homograft/Xenograft betreffenden pathologischen Befunde. Während in der ersten echokardiographischen Nachuntersuchung bei 14 Patienten (47\%) Funktionsstörungen des Homograft/Xenografts auffielen, zeigten bei der zweiten echokardiographischen Nachuntersuchung 25 Patienten (83\%) Fehlfunktionen der Neopulmonalklappe. Somit erwies sich wie in den oben zitierten Studien, dass erwartungsgemäß im postoperativen Verlauf ein PKE für die Patienten unvermeidlich ist und früher notwendig wird als eine Re-Operation des LVOT (Hörer et al. 2009). Von den Göttinger Patienten musste bei insgesamt 5 ein Austausch des Conduits in Pulmonalisposition durchgeführt werden. Bei den ausgetauschten Homografts/Xenografts handelte es sich um 2 Aortenhomografts und 3 Pulmonalis-Homografts. Da deutlich weniger Aortenhomografts als Pulmonalis-Homografts verwendet wurden (vergleiche Tabelle 3) kann eine schlechtere Haltbarkeit der Aortenhomografts vermutet werden. Allerdings ist zu bedenken, dass diese auf Grund der neuen Erkenntnis ihrer schlechteren Haltbarkeit, nur bei den länger zurückliegenden Eingriffen verwendet wurden. Da die Länge der Zeit seit der Implantation aber einen eigenen Risikofaktor für das Homograftversagen darstellt, ist anhand der Göttinger Ergebnisse die schlechtere Haltbarkeit von Aortenhomografts nicht eindeutig zu bestätigen.

Bei sehr kleinen Kindern mit entsprechend kleinen Pulmonalklappendurchmessern waren je 2 porcine (Hancock ${ }^{\circledR}$ ) und 2 bovine Xenografts (Contegra ${ }^{\circledR}$ ) verwendet worden. Diese hatten bis zum Ende des Beobachtungszeitraumes keine Re-Interventionen erfordert, so dass die sehr schlechten Ergebnisse von Williams et al. mit Frühversagen porciner Xenografts innerhalb der ersten 5 Jahre in Göttingen nicht bestätigt werden konnten. Vielmehr musste im Nachbeobachtungszeitraum kein Xenograft ausgetauscht werden. Es ist damit festzustellen, dass bei den Patienten, die einen erneuten PKE benötigten, ebenfalls die bereits in anderen Studien ermittelten Risikofaktoren wie junges Patien- 
tenalter und Verwendung eines Aortenhomografts vorlagen. Die Patientenzahl ist jedoch zu klein ist um eindeutige Aussagen treffen zu können.

Weiterhin ist festzustellen, dass erneute Eingriffe am RVOT nach Ross-Operation bereits innerhalb der ersten zehn postoperativen Jahre notwendig werden können und daher eine enge Patientenanbindung an eine nachsorgende Klinik mit regelmäßigen klinischen und echokardiographischen Nachkontrollen notwendig sind, um ein rechtzeitiges Handeln bei den mit Sicherheit auftretenden Problemen zu ermöglichen.

Nach Ross-Operation ist die regelmäßige kontinuierliche Betreuung der Patienten durch einen qualifizierten niedergelassenen Kinderkardiologen oder ein kinderkardiologisches Zentrum notwendig, da es besonders auf Ebene der PK zu relativ frühzeitigen relevanten Funktionsveränderungen kommen kann. Auch muss Wert auch auf die optimale Therapie anderer, das Herz beeinflussender Begleiterkrankungen (Einstellung des arteriellen Blutdrucks etc.) gelegt werden.

Um abschließend über die Wertigkeit der Ross-Operation für Kinder und Jugendliche urteilen zu können, müssen die Patienten weiterhin sorgfältig nachverfolgt werden. Bei guten klinischen Ergebnissen innerhalb eines Nachbeobachtungszeitraumes von bis zu 20 Jahren in der Literatur und im untersuchten Göttinger Patientenkollektiv und dem Fehlen gleichwertiger Alternativen zum AKE bei Kindern und Jugendlichen kann die RossOperation bzw. die Ross-Konno-Operation bei strenger Indikationsstellung als Methode der Wahl angesehen werden. 


\section{Zusammenfassung}

Ziel der Arbeit: Das Ziel dieser Arbeit ist die Darstellung der Ergebnisse der RossOperation in der Kinderherzchirurgie der Universitätsmedizin Göttingen als Verfahren des AKE durch die eigene Pulmonalklappe (Autograft) bei Kindern und Jugendlichen unter besonderer Berücksichtigung der Haltbarkeit und Funktion des pulmonalen Autografts und der postoperativen Entwicklung des hypertrophierten linken Ventrikels. $\mathrm{Zu}$ diesem Zwecke wurden retrospektiv klinische Daten bezüglich der linksventrikulären Funktion und Haltbarkeit des pulmonalen Autografts und der klinischen Belastbarkeit ausgewertet.

Patienten und Methoden: Untersucht wurden die Entwicklung der Neo-Aortenklappe (Autograftklappe) und des linken Ventrikels nach Ross-Operation bei 31 Patienten unter 21 Jahren, die zwischen 1994 und 2008 in Göttingen operiert wurden. Das Alter der untersuchten Patienten betrug zum Operationszeitpunkt zwischen 6 Monaten und 20 Jahren (Mittelwert: 133 Monate $\widehat{=} 11,1$ Jahre). Der Nachbeobachtungszeitraum variierte zwischen 10 Monaten und 14 Jahren (Mittelwert: 67 Monate $\widehat{=}$ 5,6 Jahre). Retrospektiv wurden 2 verfügbare postoperative Echokardiographien bezüglich der Durchmesser des Aortenklappenrings, der Aortenwurzel, des sino-tubulären Übergangs, des LVESD, des LVEDD, des IVS und der linksventrikulären HW ausgewertet. Zur Vergleichbarkeit des untersuchten Kollektivs mit gesunden Kindern und Jugendlichen wurden mit Hilfe von Regressionskurven nach Daubeney et al. (1999) und Pettersen et al. (2008) Z-Werte für die gemessenen Durchmesser bestimmt und ihre Entwicklung durch statistische Methoden ausgewertet.

Ergebnisse: Im untersuchten Patientenkollektiv konnte kein signifikanter Anstieg der Z-Werte der Durchmesser der Neo-Aortenklappe und des linken Ventrikels beobachtet werden. Die Z-Werte des Neo-Aortenwurzel-Durchmessers und des LVEDD näherten sich im Verlauf signifikant den Normkurven an.

Bei keinem Patienten wurde ein Ersatz des Autografts notwendig. 1 Patient benötigte eine operative Revision der Neo-Aortenklappe in Form einer supravalvulären Kürzung und Ummantelung der Autograft-Wurzel bzw. des sino-tubulären Übergangs. Bei 15 Patienten kam es im Verlauf zu einer Autograftinsuffizienz ersten Grades. Diese beobachteten Einschränkungen der Neo-Aortenklappenfunktion im Sinne einer geringen oder trivialen Insuffizienz waren ohne klinische Relevanz.

1 Patientin verstarb unmittelbar postoperativ an einer intrazerebralen Blutung. Zu wei- 
teren schwerwiegenden Komplikationen kam es nicht.

Die Haltbarkeit des Pulmonalis-Ersatzes durch einen Homograft oder einen Xenograft war bereits innerhalb der ersten postoperativen Dekade limitiert. Im postoperativen Nachbeobachtungszeitraum benötigten 5 Patienten einen Austausch des Implantats. Bei den ausgetauschten Homografts handelte es sich in 3 Fällen um Pulmonalis-Homografts und in 2 Fällen um Aortenhomografts.

Fazit: Die Ross-Operation ist ein Verfahren des AKE, das besonders für Kinder und Jugendliche auf Grund des Wachstumspotentials, der exzellenten Hämodynamik, der Regenerationsfähigkeit, der Infektresistenz, der uneingeschränkten natürlichen Funktion und der Athrombogenität besonders geeignet ist. Auch für Frauen mit Kinderwunsch und junge Männer mit Risikoprofil (durch Sport oder verletzungsträchtige Berufstätigkeit) oder Patienten mit Kontraindikation für eine Phenprocoumon-Therapie ist die RossOperation wegen der nicht notwendigen Marcumarisierung eine attraktive Behandlungsoption. Die befürchtete Dilatation des Autografts im Langzeitverlauf konnte nicht beobachtet werden. 


\section{Literatur}

Alexiou C, McDonald A, Langley SM, Dalrymple-Hay MJ, Haw MP, Monro JL (2000): Aortic valve replacement in children: are mechanical prostheses a good option? Eur J Cardiothorac Surg $\underline{17}$, 125-133

Bahnson HT, Spencer FC, Busse EF, Davis FW (1960): Cusp replacement and coronary artery perfusion in open operations on the aortic valve. Ann Surg 152, 94-503

Bailey CP, Glover RP, O’Neill TJ, Redondo Ramirez HP (1950): Experiences with the experimental surgical relief of aortic stenosis; a preliminary report. J Thorac Surg $\underline{20}$, 516-541

Bechtel J, Erasmi A, Misfeld M, Sievers HH (2006): Rekonstruktive Aortenklappenchirurgie: Ross-, David- und Yacoub-Verfahren. Herz $\underline{31}$, 413-422

Bekkers JA, Klieverik LM, Raap GB, Takkenberg JJ, Bogers AJ (2010): Aortic root reoperations after pulmonary autograft implantation. J Thorac Cardiovasc Surg 140, 58-63

Boethig D, Goerler H, Westhoff-Bleck M, Ono M, Daiber A, Haverich A, Breymann T (2007): Evaluation of 188 consecutive homografts implanted in pulmonary position after 20 years. Eur J Cardiothorac Surg $\underline{32}$, 133-142

Bonow RO, Carabello BA, Chatterjee K, de Leon AC, Faxon DP, Freed MD, Gaasch WH, Lytle BW, Nishimura RA, O'Gara PT et al. (2006): ACC/AHA 2006 guidelines for the management of patients with valvular heart disease: a report of the American College of Cardiology/American Heart Association task force on practice guidelines (writing committee to revise the 1998 guidelines for the management of patients with valvular heart disease) developed in collaboration with the Society of Cardiovascular Anesthesiologists endorsed by the Society for Cardiovascular Angiography and Interventions and the Society of Thoracic Surgeons. J Am Coll Cardiol 48, 1-148

Bradley LM, Midgley FM,Watson DC, Getson PR, Scott LP (1985): Anticoagulation therapy in children with mechanical prosthetic cardiac valves. Am J Cardiol 56, 533 535 
Brockbank K und Song Y (2003): Mechanisms of bioprosthetic heart valve calcification. Transplantation $\underline{75}, 1133-1135$

Brown JW, Ruzmetov M, Vijay P, Bills RG, Turrentine MW (2001): Clinical outcomes and indicators of normalization of left ventricular dimensions after Ross procedure in children. Semin Thorac Cardiovasc Surg 13, 28-34

Brown JW, Ruzmetov M, Vijay P, Rodefeld MD, Turrentine MW (2003): Surgery for aortic stenosis in children: a 40-year experience. Ann Thorac Surg 드, 1398-1411

Brown JW, Ruzmetov M, Shahriari AP, Rodefeld MD, Mahomed Y, Turrentine MW (2010): Modification of the Ross aortic valve replacement to prevent late autograft dilatation. Eur J Cardiothorac Surg 37, 1002-1007

Campbell M (1968): The natural history of congenital aortic stenosis. Br Heart J $\underline{30}$, $514-526$

Caspi J, Ilbawi MN, Roberson DA, Piccione W, Monson DO, Naja

H (1994): Extended aortic valvuloplasty for recurrent valvular stenosis and regurgitation in children. J Thorac Cardiovasc Surg 107, 1114-1120

Chambers JC, Somerville J, Stone S und Ross DN (1997): Pulmonary autograft procedure for aortic valve disease: long-term results of the pioneer series. Circulation $\underline{96}$, 2206-2214

Daubeney PEF, Blackstone EH, Weintraub RG, Slavik Z, Scanlon J, Webber SA (1999): Relationship of the dimension of cardiac structures to body size: an echocardiographic study in normal infants and children. Cardiol Young $\underline{9}, 402-410$

Duebener LF, Stierle U, Erasmi A, Bechtel MF, Zurakowski D, Bohm JO, Botha CA, Hemmer W, Rein JG, Sievers HH (2005): Ross procedure and left ventricular mass regression. Circulation $\underline{112}$, I, 415-422 
Elkins R, Thompson D, Lane M, Elkin C, Peyton M (2008): Ross operation: 16-year experience. J Thorac Cardiovasc Surg 통, 623-630

Gilbert JW, Morrow AG und Braunwald E (1960): Results of Open Commissurotomy in Acquired Calcific Aortic Stenosis: Clinical and Hemodynamic Studies in Patients Operated upon With General Hypothermia. Ann Surg 151, 1-10

Hanke T, Stierle U, Boehm JO, Botha CA, Matthias Bechtel JF, Erasmi A, Misfeld M, Hemmer W, Rein JG, Robinson DR et al. (2007): Autograft regurgitation and aortic root dimensions after the Ross procedure: the German Ross Registry experience. Circulation 116, I, 251-258

Hawkins JA, Minich LL, Shaddy RE, Tani LY, Orsmond GS, Sturtevant JE, Mc- Gough EC (1996): Aortic valve repair and replacement after balloon aortic valvuloplasty in children. Ann Thorac Surg 61, 1355-1358

Henry WL, Ware J, Gardin JM, Hepner SI, McKay J, Weiner M (1978): Echocardiographic measurements in normal subjects. Growth-related changes that occur between infancy and early adulthood. Circulation 57, 278-285

Hokken RB, Bogers AJ, Taams MA, Willems TP, Cromme-Dijkhuis AH, Witsenburg M, Spitaels SE, van Herwerden LA, Bos E (1995): Aortic root replacement with a pulmonary autograft. Eur J Cardiothorac Surg $\underline{9}, 378-383$

Hokken RB, Bartelings MM, Bogers AJ, Gittenberger-de Groot AC (1997a): Morphology of the pulmonary and aortic roots with regard to the pulmonary autograft procedure. J Thorac Cardiovasc Surg 113, 453-461

Hokken RB, Bogers AJ, Taams MA, Schiks-Berghourt MB, van Herwerden LA, Roelandt JR, Bos E (1997b): Does the pulmonary autograft in the aortic position in adults increase in diameter? An echocardiographic study. J Thorac Cardiovasc Surg 113, 667-674

Hokken RB, Cromme-Dijkhuis AH, Bogers AJ, Spitaels SE, Witsenburg M, Hess J, Bos E (1997c): Clinical outcome and left ventricular function after pulmonary autograft implantation in children. Ann Thorac Surg $\underline{63}, 1713-1717$ 
Hörer J, Hanke T, Stierle U, Takkenberg J, Bogers A, Hemmer W, Rein JG, Hetzer R, Hübler M, Robinson D R (2009): Neoaortic root diameters and aortic regurgitation in children after the ross operation. Ann Thorac Surg 88, 594 - 600

Hörer J, Stierle U, Bogers A, Rein J, Hetzer R, Sievers H, Lange R (2010): Reinterventions on the autograft and the homograft after the ross operation in children. Eur $\mathrm{J}$ Cardiothorac Surg 37, 1008 - 1014

Iturbe-Alessio I, Fonseca MC, Mutchinik O, Santos MA, Zajarias A, Salazar E (1986): Risks of anticoagulant therapy in pregnant women with arti cial heart valves. N Engl J Med 315, 1390-1393

Javadpour H, Veerasingam D und Wood AE (2002): Calci cation of homograft valves in the pulmonary circulation - is it device or donation related? Eur J Cardiothorac Surg 22, 78-81

Jonas $\mathrm{R}$ (2005): The ross procedure is not the procedure of choice for the teenager requiring aortic valve replacement. Semin Thorac Cardiovasc Surg: Pediatr Card Surg Ann $\underline{8}, 176-180$

Justo RN, McCrindle BW, Benson LN, Williams WG, Freedom RM, Smallhorn JF (1996): Aortic valve regurgitation after surgical versus percutaneous balloon valvotomy for congenital aortic valve stenosis. Am J Cardiol 77, 1332-1338

Kadner A, Raisky O, Degandt A, Tamisier D, Bonnet D, Sidi D, Vouhe PR (2008): The Ross procedure in infants and young children. Ann Thorac Surg $\underline{85}$, 803-808

Konno S, Imai Y, Iida Y, Nakajima M, Tatsuno K (1975): A new method for prosthetic valve replacement in congenital aortic stenosis associated with hypoplasia of the aortic valve ring. J Thorac Cardiovasc Surg 70, 909-917

Koren MJ, Devereux RB, Casale PN, Savage DD, Laragh JH (1991): Relation of left ventricular mass and geometry to morbidity and mortality in uncomplicated essential hypertension. Ann Intern Med 114, 345-352 
Kotzot D, Bernasconi F, Brecevic L, Robinson WP, Kiss P, Kosztolanyi G, Lurie IW, Superti-Furga A, Schinzel A (1995): Phenotype of the Williams-Beuren syndrome associated with hemizygosity at the elastin locus. Eur J Pediatr 154, 477-482

Kouchoukos N, Masetti P, Nickerson N, Castner C, Shannon W, Davila-Roman V (2004): The ross procedure: Long-term clinical and echocardiographic follow-up. Ann Thorac Surg $\underline{78}, 773-781$

Kusa J, Biakowski J und Szkutnik M (2004): Percutaneous balloon aortic valvuloplasty in children: early and long-term outcome. Kardiol Pol 60, 48-56

LeMaire SA und Coselli JS (1997): Aortic root surgery in Marfan syndrome: current practice and evolving techniques. J Card Surg $\underline{12}$, 137-141

Marino BS, Wernovsky G, Rychik J, Bockoven JR, Godinez RI, Spray TL (1999): Early results of the Ross procedure in simple and complex left heart disease. Circulation $\underline{100}$, I, $162-166$

Matsuki O, Okita Y, Almeida R, McGoldrick J, Hooper TL, Robles A, Ross DN (1988): Two decades' experience with aortic valve replacement with pulmonary autograft. J Thorac Cardiovasc Surg $\underline{95}$, 705-711

McCrindle BW, Blackstone EH, Williams WG, Sittiwangkul R, Spray TL, Azakie A, Jonas RA (2001): Are outcomes of surgical versus transcatheter balloon valvotomy equivalent in neonatal critical aortic stenosis? Circulation 104, I, 152-158

McElhinney DB, Lock JE, Keane JF, Moran AM, Colan SD (2005): Left heart growth, function, and reintervention after balloon aortic valvuloplasty for neonatal aortic stenosis. Circulation $\underline{111}, 451-458$

Moidl R, Simon P, Aschauer C, Chevtchik O, Kupilik N, Rodler S, Wolner E, Laufer G (2000): Does the ross operation full the objective performance criteria established for new prosthetic heart valves? J Heart Valve Dis $\underline{9}$, 190-194 
Moinuddeen K, Quin J, Shaw R, Dewar M, Tellides G, Kopf G, Elefteriades J (1998): Anticoagulation is unnecessary after biological aortic valve replacement. Circulation $\underline{98}$, I, 95-98

Neves JP, Gulbenkian S, Ramos T, Martins AP, Caldas MC, Mascarenhas R, Guerreiro M, Matoso-Ferreira A, Santos R, Monteiro C et al. (1997): Mechanisms underlying degeneration of cryopreserved vascular homografts. J Thorac Cardiovasc Surg 113, 1014-1021

Niwaya K, Knott-Craig CJ, Lane MM, Chandrasekaren K, Overholt ED, Elkins RC (1999): Cryopreserved homograft valves in the pulmonary position: risk analysis for intermediate-term failure. J Thorac Cardiovasc Surg 117, 141-146

Ohye RG, Gomez CA, Ohye BJ, Goldberg CS, Bove EL (2001): The Ross/Konno procedure in neonates and infants: intermediate-term survival and autograft function. Ann Thorac Surg 72, 823-830

Pasquali S, Cohen M, Shera D, Wernovsky G, Spray T, Marino B (2007a): The relationship between neo-aortic root dilation, insufficiency, and reintervention following the ross procedure in infants, children, and young adults. J Am Coll Cardiol $\underline{49}$

Pasquali SK, Shera D, Wernovsky G, Cohen MS, Tabbutt S, Nicolson S, Spray TL, Marino BS (2007b): Midterm outcomes and predictors of reintervention after the Ross procedure in infants, children, and young adults. J Thorac Cardiovasc Surg 133, 893-899

Pedra CA, Sidhu R, McCrindle BW, Nykanen DG, Justo RN, Freedom RM, Benson LN (2004): Outcomes after balloon dilation of congenital aortic stenosis in children and adolescents. Cardiol Young 14, 315-321

Peterseim DS, Cen YY, Cheruvu S, Landolfo K, Bashore TM, Lowe JE, Wolfe WG, Glower DD (1999): Long-term outcome after biologic versus mechanical aortic valve replacement in 841 patients. J Thorac Cardiovasc Surg 117, 890 - 897

Pettersen MD, Du W, Skeens ME, Humes RA (2008): Regression equations for calculation of z scores of cardiac structures in a large cohort of healthy infants, children, and adolescents: an echocardiographic study. J Am Soc Echocardiogr 21, 922-934 
Pibarot P, Dumesnil JG (2000): Hemodynamic and clinical impact of prosthesispatient mismatch in the aortic valve position and its prevention. J Am Coll Cardiol 36, 1131-1141

Presbitero P, Somerville J, Revel-Chion R, Ross D (1982): Open aortic valvotomy for congenital aortic stenosis. Late results. Br Heart J $\underline{47}, 26-34$

Rastan H und Koncz J (1975): [Plastic enlargement of the left ventricular out ow tract. A new operative method (author's transl)]. Thoraxchir Vask Chir 23, 169-175

Reddy VM, Rajasinghe HA, Teitel DF, Haas GS, Hanley FL (1996): Aortoventriculoplasty with the pulmonary autograft: the "Ross-Konno" procedure. J Thorac Cardiovasc Surg $\underline{111}, 158-165$

Roman M, Devereux R, Kramer-Fox R, O'Loughlin J (1989): Two-dimensional echocardiographic aortic root dimensions in normal children and adults. Am J Cardiol $\underline{64}, 507$ $-512$

Ross D (1967a): Replacement of aortic and mitral valves with a pulmonary autograft. Lancet $\underline{290}, 956$ - 958

Ross DN (1967b): Homograft replacement of the aortic valve. Br J Surg $\underline{54}, 842-845$

de Sa M, Moshkovitz Y, Butany J, David TE (1999): Histologic abnormalities of the ascending aorta and pulmonary trunk in patients with bicuspid aortic valve disease: clinical relevance to the ross procedure. J Thorac Cardiovasc Surg 118, 588-594

Schmid FX, Keyser A, Djavidani B, Link J, Holmer S, Birnbaum DE (2002): Left ventricular remodeling after pulmonary autograft aortic valve replacement: evaluation with color-Doppler echocardiography and magnetic resonance imaging. Artif Organs $\underline{26}, 444-$ 448

Schumacher G: Obstruktionen im Bereich des linken Herzens. In: Schumacher G, Hess J, Bühlmeyer K: Klinische Kinderkardiologie; Diagnostik und Therapie der angeborenen Herzfehler. 4. Auflage. Springer Verlag, Heidelberg 2008 
Sholler GF, Keane JF, Perry SB, Sanders SP, Lock JE (1988): Balloon dilation of congenital aortic valve stenosis. Results and influence of technical and morphological features on outcome. Circulation $\underline{78}, 351-360$

Sievers HH, Stierle U, Schmidtke K (2004): 1. Jahresbericht des deutschen Rossregisters. Eigendruck Lübeck

Smith JC (1967): The pathology of human aortic valve homografts. Thorax $\underline{22}, 114-138$

Snider AR, Enderlein MA, Teitel DF, Juster RP (1984): Two-dimensional echocardiographic determination of aortic and pulmonary artery sizes from infancy to adulthood in normal subjects. Am J Cardiol $\underline{53}, 218-224$

Solymar L, Sudow G, Holmgren D (2000): Increase in size of the pulmonary autograft after the Ross operation in children: growth or dilation? J Thorac Cardiovasc Surg 119, 4-9

van Son JA, Falk V, Mohr FW (1997): Ross-Konno operation with resection of endocardial fibroelastosis for critical aortic stenosis with borderline-sized left ventricle in neonates. Ann Thorac Surg $\underline{63}, 112-116$

Stewart RD, Backer CL, Hillman ND, Lundt C und Mavroudis C (2007): The Ross operation in children: effects of aortic annuloplasty. Ann Thorac Surg 모, 1326-1330

Swan L, Wilson N, Houston A, Doig W, Pollock J und Hillis W (1998): The long-term management of the patient with an aortic coarctation repair. Eur Heart J 19, 382-386 
Swann WK, Bradsher JT, Rodriguez-Arroyo J (1954): The surgical treatment of aortic stenosis. South Med J 느, 1067-1069

Takkenberg JJ, Kappetein AP, van Herwerden LA, Witsenburg M, Van Osch-Gevers L, Bogers AJ (2005): Pediatric autograft aortic root replacement: a prospective follow-up study. Ann Thorac Surg 으, 1628-1633

Tirilomis T, Coskun KO, Popov AF, Ruschewski W (2013): Aortic surgery after previous procedure of congenital aortic stenosis. Artif Organs $\underline{37}, 92-96$

Valeske K, Muller M, Hijjeh N, Bauer J, Boning A, Schranz D, Akinturk H (2010): The fate of the pulmonary autograft in the aortic position: experience and results of 98 patients in twelve years. Thorac Cardiovasc Surg $\underline{58}$, 334-338

Walters HL, Lobdell KW, Tantengco V, Lyons JM, Hudson CL, Struble SL, Hakimi M (1997): The Ross procedure in children and young adults with congenital aortic valve disease. J Heart Valve Dis $\underline{6}, 335-342$

Williams IA, Quaegebeur JM, Hsu DT, Gersony WM, Bourlon F, Mosca RS, Gersony DR, Solowiejczyk DE (2005): Ross procedure in infants and toddlers followed into childhood. Circulation 112, I, 390-395

Wong V, Cheng CH, Chan KC (1993): Fetal and neonatal outcome of exposure to anticoagulants during pregnancy. Am J Med Genet $\underline{45}, 17-21$

Zilberman M, Khoury P, Kimball R (2005): Two-dimensional echocardiographic valve measurements in healthy children: Gender-specific differences. Pediatr Cardiol 26, 356360 